\title{
STUDY OF STATISTICAL AND COMPUTATIONAL INTELLIGENCE METHODS OF DETECTING TEMPORAL SIGNATURE OF FOREST FIRE HEAT PLUME FROM SINGLE-BAND GROUND-BASED INFRARED VIDEO
}

\author{
A Thesis \\ presented to \\ the Faculty of California Polytechnic State University, \\ San Luis Obispo \\ By \\ Daniel G. Kohler \\ June 2012 \\ In Partial Fulfillment \\ of the Requirements for the Degree \\ Master of Science in Electrical Engineering
}


(C) 2012

Daniel G. Kohler

ALL RIGHTS RESERVED 


\section{COMMITTEE MEMBERSHIP}

TITLE:

AUTHOR:

DATE SUBMITTED:

COMMITTEE CHAIR:

COMMITTEE MEMBER:

COMMITTEE MEMBER:
STUDY OF STATISTICAL AND COMPUTATIONAL

INTELLIGENCE METHODS OF DETECTING

TEMPORAL SIGNATURE OF FOREST FIRE HEAT

PLUME FROM SINGLE-BAND GROUND-BASED

INFRARED VIDEO

Daniel G. Kohler

March 2012

Dr. John Saghri, Associate Professor

Dr. John Jacobs, Raytheon Professor of Practice

Dr. Jane Zhang, Associate Professor 


\begin{abstract}
This thesis will analyze video from land-based, cooled mid-wave infrared cameras to identify temporal features indicative of a heat plume from a forest fire. Desirable features and methods will show an ability to distinguish between heat plume movement and other movements, such as foliage, vehicles, humans, and birds in flight. Features will be constructed primarily using combinations of statistics and principal component analysis (PCA) with intent to detect key characteristics of fire and heat plume: persistence and growth. Several classification systems will combine and filter the features in an attempt to classify pixels as either heat or non-heat. The classification systems will be tuned and compared with common metrics of error rate and computation time. It was found that the movement pattern of a heat plume could be distinguished from the similar movement pattern of foliage by detecting outlier movement patterns, a phenomenon associated with the growth property of fire. Outlier movement patterns were best detected by thresholding the quotient of mean and median of a set of variance measurements over time. The best tested classifier in terms of minimizing false positives without losing the heat signal came from PCA of a dual-range moving average difference.
\end{abstract}

Keywords: Forest Fire Smoke Heat Temporal Detection Features Classification 


\section{ACKNOWLEDGEMENTS}

Dr. Saghri and Dr. Jacobs offered their insights and experience throughout this entire project. It wouldn't be what it is without their dedication and passion.

Thank you to the Raytheon and FLIR employees who generously volunteered their weekend time to configure and facilitate the data collection mission: Steve Botts, Marc Bauer, Chris Tracy, Dana Schneider.

Thank you to Gary Hughes from FLIR, who offered continuing hardware support and engineering expertise. 


\section{TABLE OF CONTENTS}

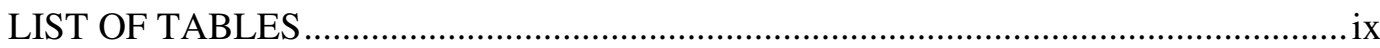

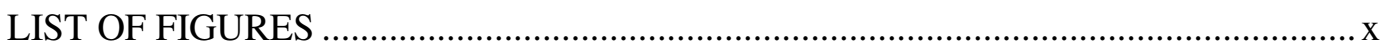

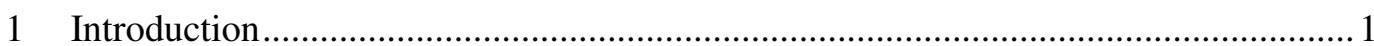

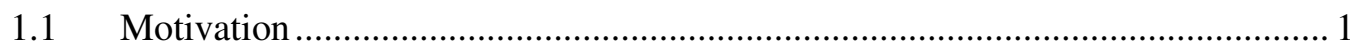

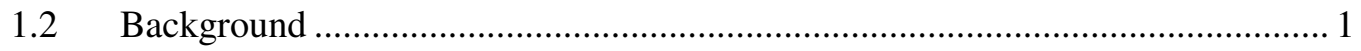

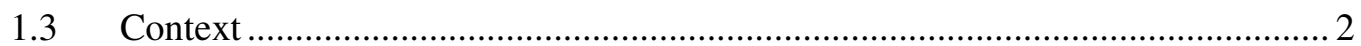

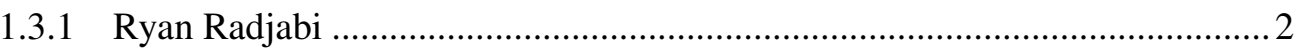

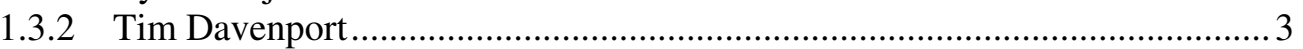

1.3.3 George Moussa .................................................................................... 3

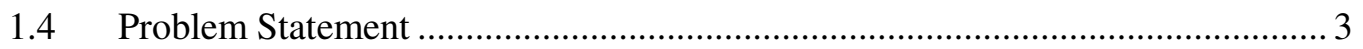

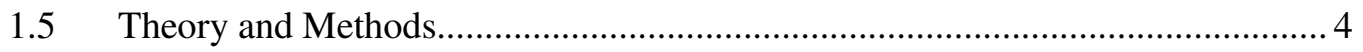

1.6 Document Organization and Notes to the Reader ................................................. 6

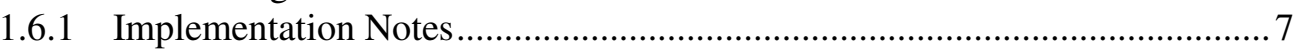

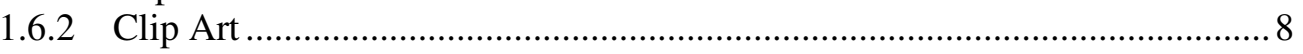

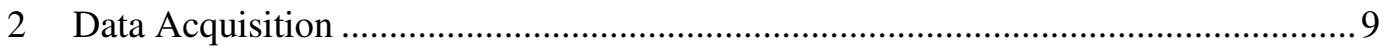

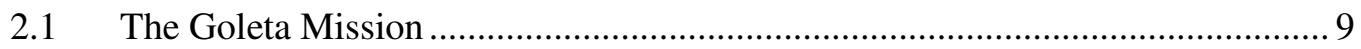

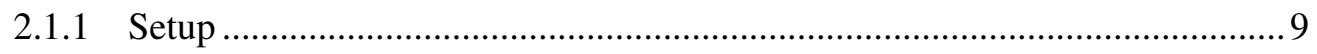

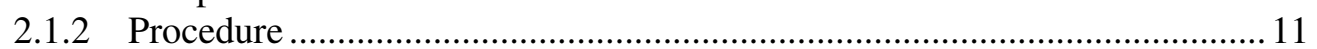

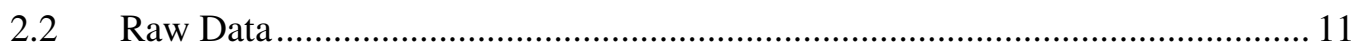

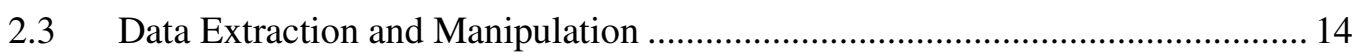

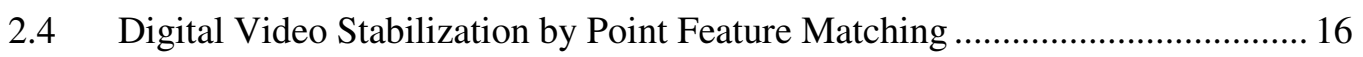

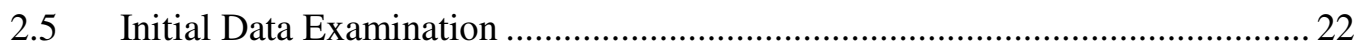

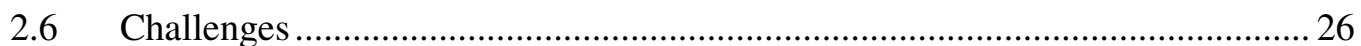

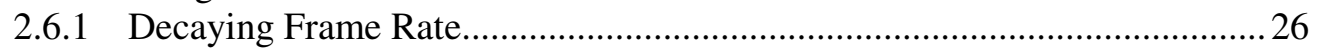

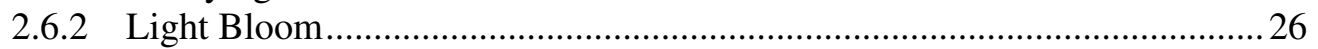

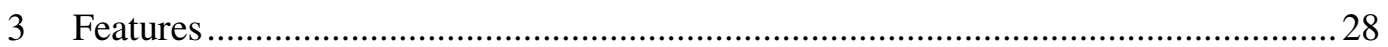

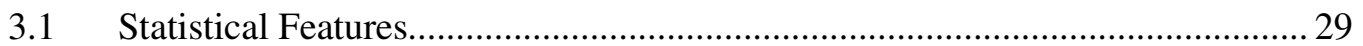

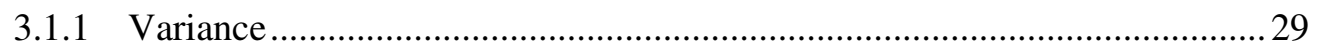

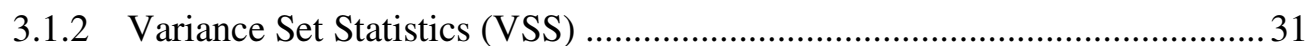

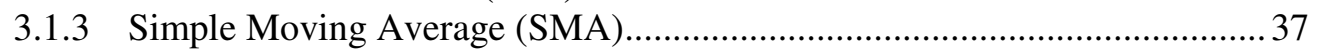

3.1.4 Exponentially Weighted Moving Average (EWMA or EMA) ..................... 37

3.1.5 Dual-Range Moving Average Difference (DRMAD) ................................. 39

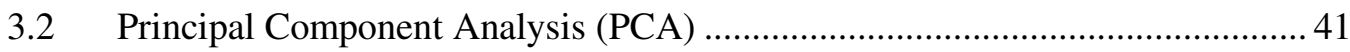

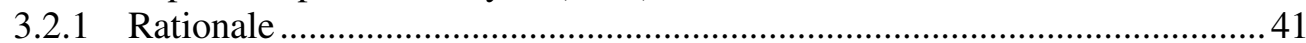

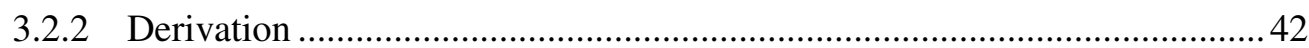

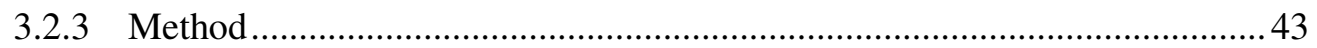




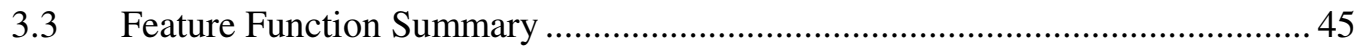

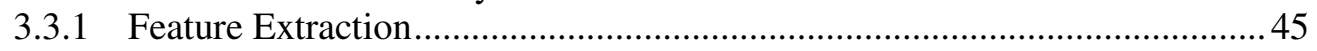

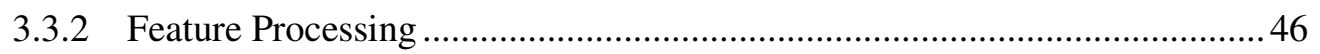

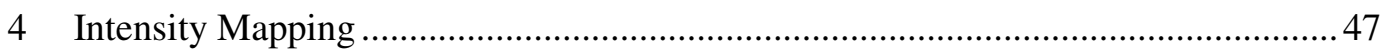

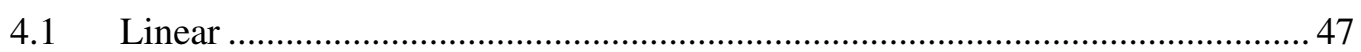

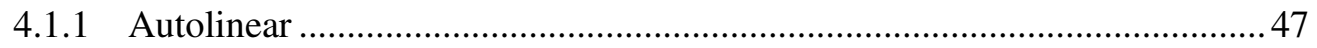

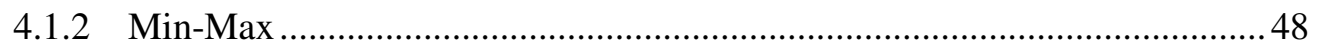

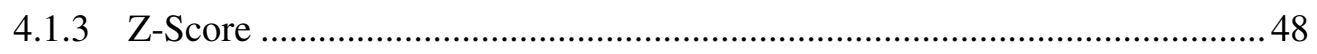

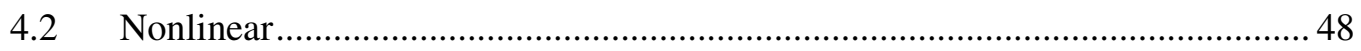

4.2.1 Gamma Mapping (Power Law) ….......................................................... 48

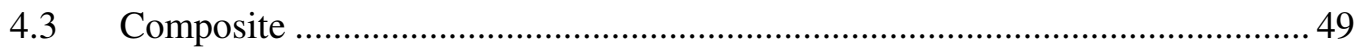

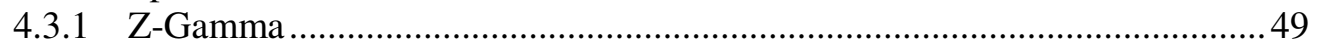

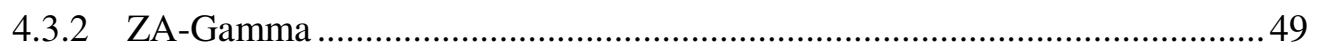

4.4 Intensity Mapping Function Summary …….................................................... 50

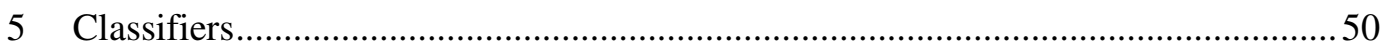

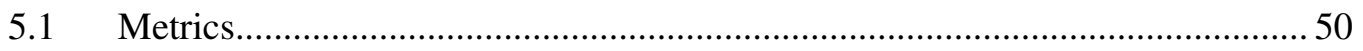

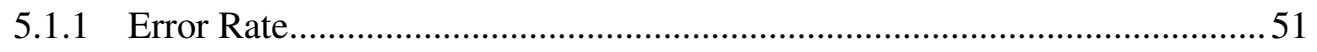

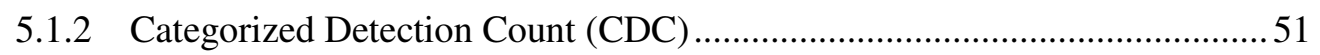

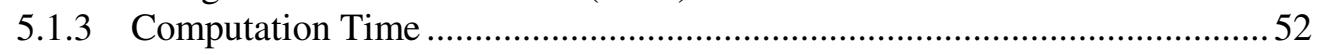

5.2 Variance Corridor / Bayesian Classifier.......................................................... 52

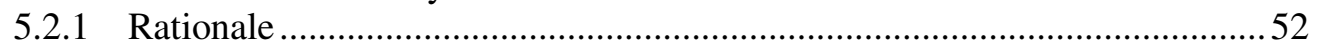

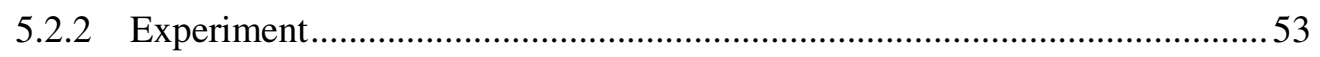

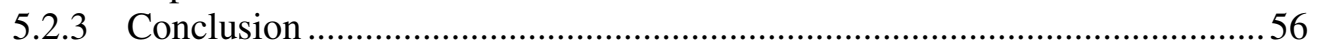

5.3 Variance Set Statistics - Mean and Max Thresholding (MMT) .........................56

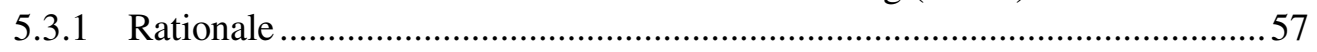

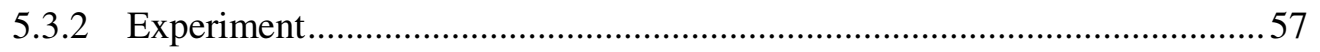

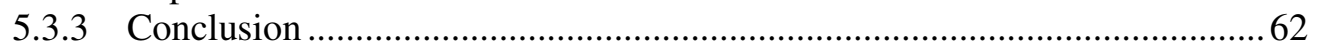

5.4 Variance Set Statistics - Mean by Median (MBM) Quotient .............................6 63

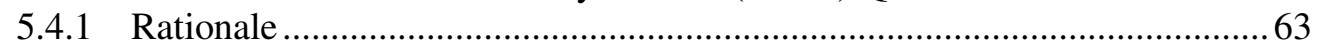

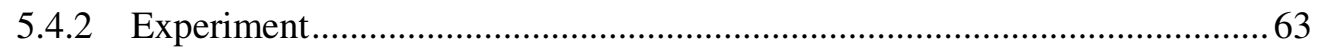

5.4.3 Variance Augmented Mean-By-Median Quotient (VAMBM) ......................67

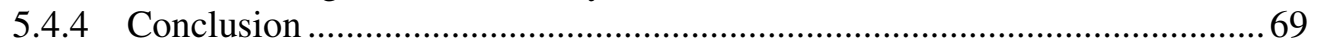

5.5 Principal Component Analysis of Dual-Range Moving Average

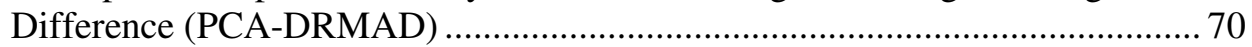

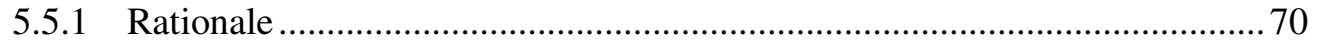

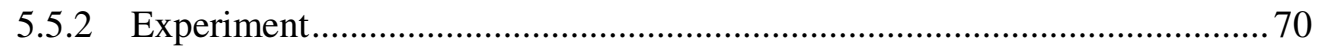

5.5.3 Principle Component Image Combination................................................... 75

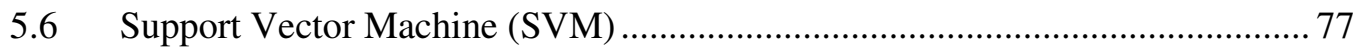

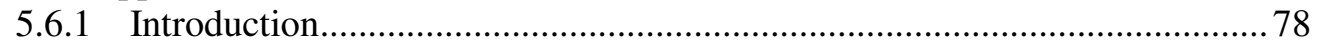

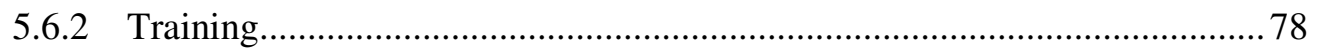

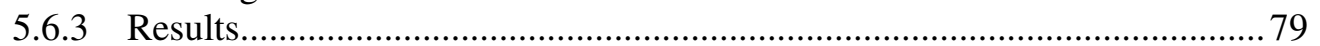

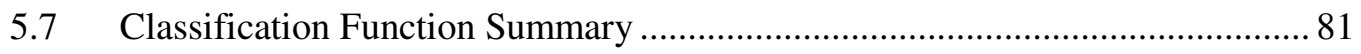

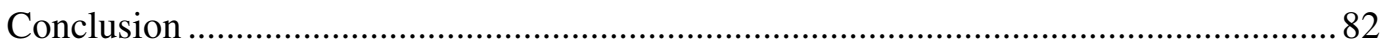




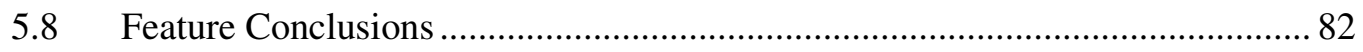

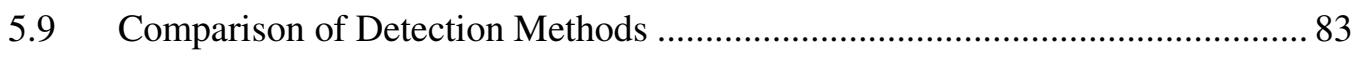

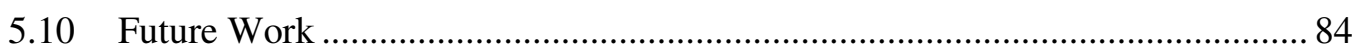

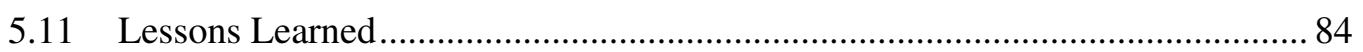

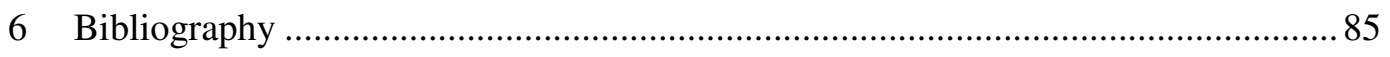

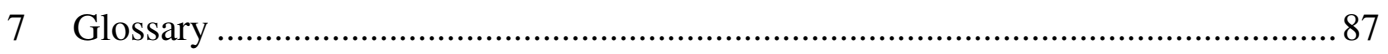

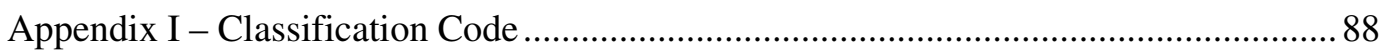




\section{LIST OF TABLES}

Table 1 - Generic Format for Function Description Tables within This

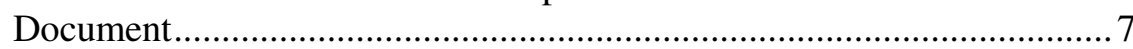

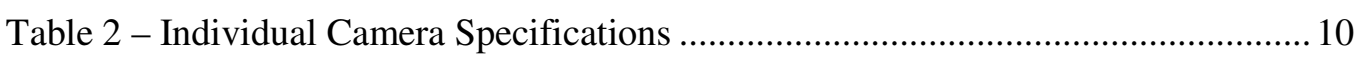

Table 3 - Common Camera Specifications ................................................................. 10

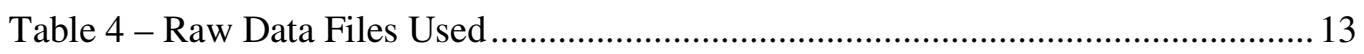

Table 5 - Data Acquisition and Manipulation Function Descriptions ............................ 16

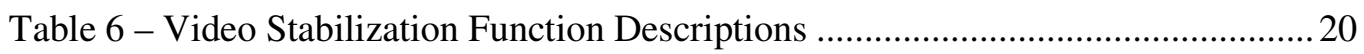

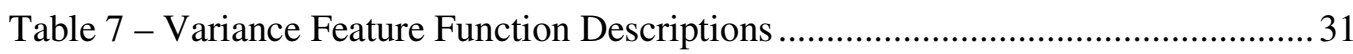

Table 8 - Variance Set Statistics Defined for Figure 18 Masks (Statistics from oats1 video, frames. Standard deviation shown for

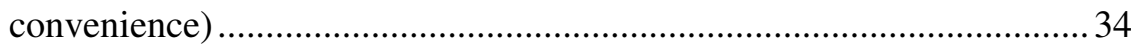

Table 9 - More Variance Set Statistics (From pre-stabilized pine_cones

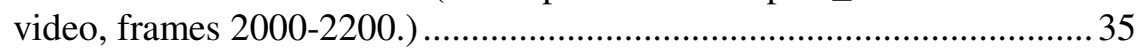

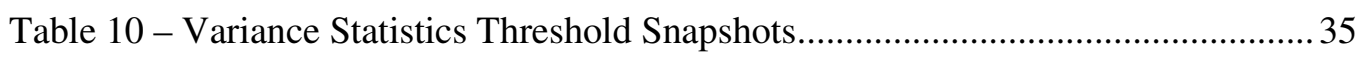

Table 11 - Feature Extraction Function Descriptions ...................................................... 45

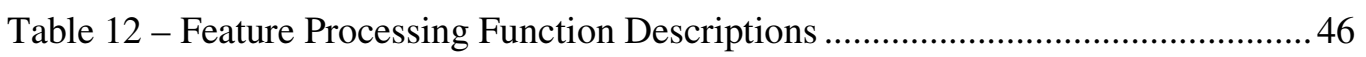

Table 13 - Intensity Mapping Function Descriptions ...................................................50

Table 14 - Correspondence Between Bin Center and Class with Largest

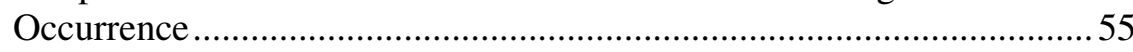

Table 15 - Variance Set Statistics Thresholds (sequence length 30, set size 20) .59

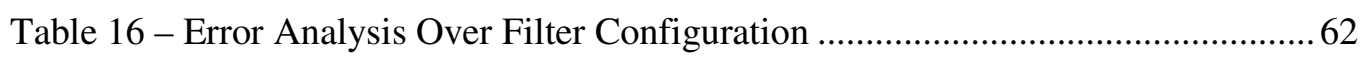

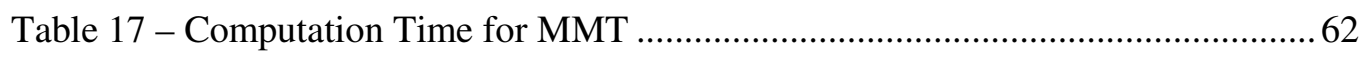

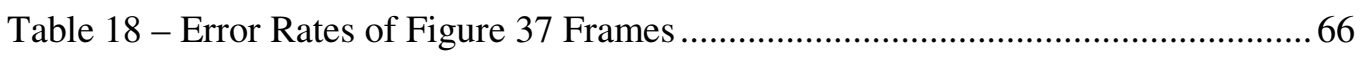

Table 19 - Error Rate of MBM_var30 Over VSS Configurations..................................6 68

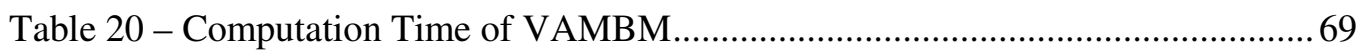

Table 21 - PCA-DRMAD Difference Ranges (Cases) .................................................. 71

Table 22 - Error Analysis for PCA-DRMAD (Difference Case 1) ................................. 74

Table 23 - Error Analysis for All PCA-DRMAD Difference

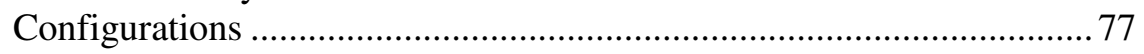

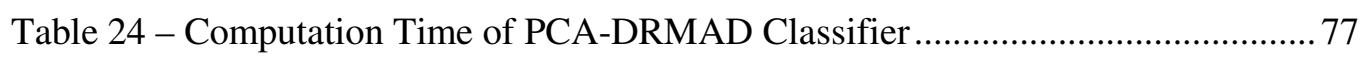

Table 25 - Classifier Sensitivity - Categorized Detection Count................................... 83

Table 26 - Comparison of Classifier Computation Time ……..................................... 83 


\section{LIST OF FIGURES}

Figure 1 - Typical Frame in Scene Showing Region of Interest, Containing

Active Fire (pine_cones channel, frame 2000) ........................................

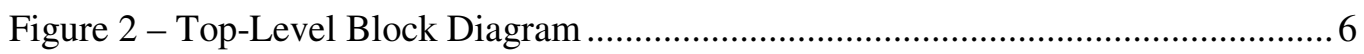

Figure 3 - Data Collection Setup Locations-of-Interest ............................................... 10

Figure 4 - 14-Bit Little-Endian Raw Data Saved in 16-Bit Word ................................. 12

Figure 5 - Cooled Infrared Data Path ........................................................................ 12

Figure 6 - Raw Data File Frame-Wise Separated ...................................................... 14

Figure 7 - Frames Converted from (x, y) Order to (r, c) Order ..................................... 15

Figure 8 - Corner Points Detected By FAST Algorithm ................................................ 18

Figure 9 - Correspondences Between Corner Points ....................................................... 19

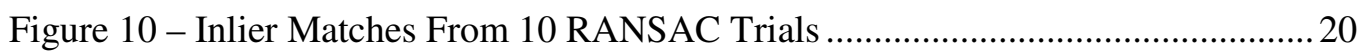

Figure 11 - Variance Frames Before and After Stabilization (pine_cones channel, frames 2000 through 2200) ..................................................... 21

Figure 12 - Frame 2392 of pine_cones (not stabilized) ................................................. 23

Figure 13 - Close-Up of Barbecue Pit with Intensity Values Shown .............................24

Figure 14 - Mean and Standard Deviation Per-Pixel of Barbecue Pit ............................25

Figure 15 - Relationship between Frame Group and Feature Frame.............................2 29

Figure 16 - Variance Feature Frames Sample (Stabilized oats1 channel,

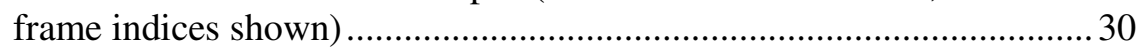

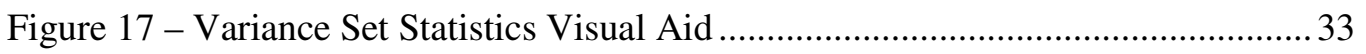

Figure 18 - Mask Locations for Data Sampling ............................................................ 34

Figure 19 - Time Sequences of Pixels by Region. Top/Red: Figure 18(c). Middle/Green: Figure 18(b). Bottom/Blue: Figure 18(e) ....................... 36

Figure 20 - Comparison of Frequency Response Magnitude of SMA and

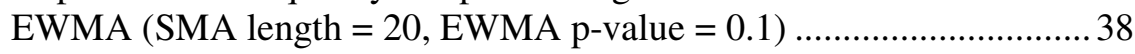

Figure 21 - Comparison of SMA and EWMA on Step Function (SMA

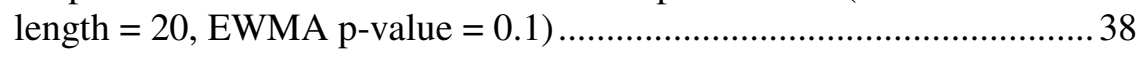

Figure 22 - Comparison of Dual Range Exponentially Weighted Moving Average Difference (DRMAD) pairs (oats1 stabilized channel, e.g. lower left pane is EWMA of p-value $=0.1$ less EWMA of $\mathrm{p}$-value $=0.02$ )

Figure 23 - Example of PCA (b) on Input Frames (a) from oats1 DRMAD (EWMA0.07-0.02_PCA6_autolinear, non-uniform linear map per PC) 
Figure 24 - Gamma Mapping Example Transfer Function and Histograms

Figure 25 - Movement Category Masks .....

Figure 26 - Exponentially Binned Variance Histogram Per Masked Region (exponentially indexed abscissa: $10^{\wedge} \mathrm{x}$ ) ....

Figure 27 - Result of Variance Corridor / Bayesian Variance Classification

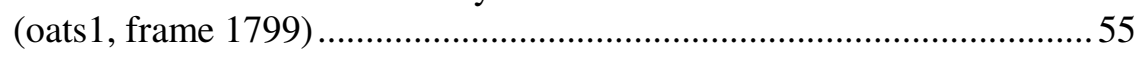

Figure 28 - Variance within Heat Plume of Figure 27 ...............................................56

Figure 29 - varMean and varMax from oats1, Frame 3140 (Contrast

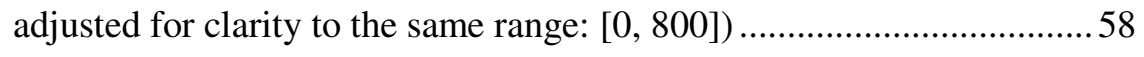

Figure 30 - varMean in the Heat Plume, Frame 3140 .................................................5

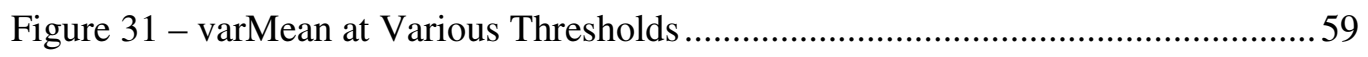

Figure 32 - Intersection of Thresholded VSS Mean and Max for Different

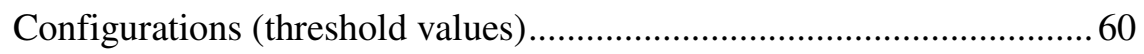

Figure 33 - Comparison of Filter Lengths on varStats_loose Configuration.................. 61

Figure 34 - Categorized Detection Count Per Channel of VSL for Several

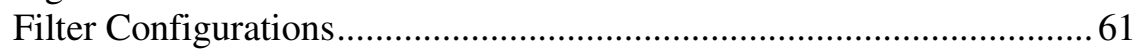

Figure 35 - Comparison of Feature Quotients ............................................................... 64

Figure 36 - Comparison of VSS parameters on MBM ……......................................... 65

Figure 37 - Comparison of VSS Parameters on MBM, Thresholded, and Median Filtered.

Figure 38 - Comparison of VSS Parameters for VAMBM Data (variance length 30)

Figure 39 - Categorized Detection Count Per Channel of MBM_var30

(VAMBM) for Several VSS Configurations .68

Figure 40 - PCA-DRMAD with Automatic Threshold ................................................ 73

Figure 41 - CDC Comparing Auto-Thresholded PC Images (Difference Case 1)

Figure 42 - CDC Comparing Classifiers for All PCA-DRMAD Difference Cases.

Figure 43 - Comparison of Classifiers for All PCA-DRMAD Difference Configurations . .76

Figure 44 - Train_var30 Mask, "Heat Mask" (frames 3030 - 3230, step 10). .78

Figure 45 - Support Vector Machine Output 80 


\section{INTRODUCTION}

\subsection{Motivation}

Forest fires threaten lives and property. The past 10 years from 2002 to 2012 in California have officially seen 26,128 fires, affecting an average of 971,000 acres per year [1]. Early detection of forest fires would lead to an earlier deployment of fire fighting resources, which could preclude damage. In the interest of saving lives and property, this thesis examines methods for use in detection of forest fire by infrared video.

\subsection{Background}

The simplest method of early forest fire detection is to post a diligent human observer at an effective vantage point, and instruct the observer to raise an alarm when they see fire or smoke. Thankfully, modern technology provides many useful alternatives. The most ubiquitous fire detection system, as seen in buildings, relies on a device on a wall that detects smoke, heat, or carbon monoxide, indicators of combustion [2]. This detection scheme is referred to as "point detection." Unfortunately, it only works well in an enclosed environment, in somewhat close proximity. Another category is "beam detection," which uses cameras or lasers to monitor an enclosed space. Though longer-range, they can suffer from indirect sunlight at some times of day, or from misalignment, perturbation, or dirty lenses [3]. There's no school like the old school, and a group from the University of Nevada, Reno are blending old and new by installing a remote 360-degree, solar powered camera that streams video to a public website, where thousands of volunteers can check on it and manually raise an alarm [18].

Satellite solutions can work, but they have their own limitations. They can suffer from atmospheric conditions and coarse resolution in space and time. For example, the well-known MODIS algorithm, the subject of many current papers, implemented on the TERRA satellite and 
launched in 1999, has a spatial resolution of $250 \mathrm{~m}$ : hardly suitable for detecting small forest fires [19]. Satellite systems are better suited to conservative fire detection algorithms that detect 30$40 \%$ of fires that account for $80-99 \%$ of burned biomass, according to a study on forest fires in Brazil [20]. It appears that only ground-based systems can detect fires early enough for tactical response.

Many papers address the subject of smoke detection and fire detection. Of those that consider detection from video sources, the trend veers towards considering multiple dimensions of the event, in spatial, temporal, and spectral domains [4], [5], [6]. More domains and more features, if combined wisely, can add robustness to the system. Unfortunately, these come at the cost of increased sensing system requirements. If an adequate system could be demonstrated to detect smoke or heat plume from a single camera, it would reduce cost and complexity, be more easily maintained, more light-weight, and more widely accessible.

\subsection{Context}

This thesis explores just a few aspects of an on-going and wide-spanning inquiry into early forest fire detection under Professor Saghri. Other Master's theses and senior projects have explored related topics.

\subsubsection{Ryan Radjabi}

Applies multi-temporal principal component analysis (PCA) with nonlinear mapping on mid-wave infrared video to identify fire and heat plume activity.

Ryan did his project first, so he pioneered some of the data manipulation and processing techniques. 


\subsubsection{Tim Davenport}

Applies multi-temporal and multi-spectral principal component analysis (PCA) on blue, green, red, mid-wave, and long-wave IR, then applies texture analysis to identify heat plume activity and raise alarm.

Tim refined and expanded Ryan's work in terms of data manipulation, and the breadth and complexity of processing techniques. Some of the lowest-level data manipulation code of this thesis is based on Tim's, and has been modified and further expanded.

\subsubsection{George Moussa}

George performs texture and motion analysis in the visible spectrum, in time- and frequency-domain. At the time this paper was written, George was in the early stages of his research.

\subsection{Problem Statement}

Given infrared video footage of a scene containing an active barbecue pit at mid-day, study features that indicate the presence of heat plume. Heat plume is the warmth that rises from a combustion source, as measured by an infrared camera. Heat plume should not be confused with smoke plume, which is detected in the visible domain due to scattered light as a result of particulate by-products of combustion. Design a system that detects the heat plume and raises an alarm. The available infrared channels include cooled mid-wave (CMWIR), cooled long-wave (CLWIR), and uncooled long-wave (UCLWIR).

An active flame is trivially simple to detect with an intensity threshold, so this problem does not include flame detection, but rather focuses on the heat rising from the flame, which has a higher probability of showing up in line-of-sight than the flame itself, by virtue of its relative size. Infrared can detect heat from a fire by day or night, making a more robust solution. 


\subsection{Theory and Methods}

To detect heat plume, a system must know the characteristics of the heat plume, and those of the background. Assume that heat plumes come from fire. Then heat plumes share some noteworthy characteristics of fire, specifically, persistence and growth [13]. In the case of fire, persistence and growth can be measured by pixel intensity over time, in visible or infrared. However, a fire can be considered a point source, where the heat plume emerges from that point source, which this thesis assumes is obscured. Furthermore, the heat of the fire pixel would dominate that pixel's DC component, where the heat plume wavers and simply adds to the natural background intensity of the pixels it passes through. These two points imply that persistence and growth can't be measured by intensity, but rather by movement. Relying on movement creates a challenge: many elements of a scene move in patterns very similar to that of the heat plume. People walking and cars moving pose a simple challenge, but by far the most difficult phenomenon to distinguish from heat plume has been the foliage, because both heat plumes and foliage move and vary in the wind.

This thesis will attempt to identify features that indicate persistence and growth, and incorporate those features into a classifying system that detects the eruption of a heat plume. A standardized system of measuring error rate will compare different classification systems.

The features will derive from statistics employed in such a way as to represent timerelevant information. These statistics will consist of variances, weighted means, medians, thresholds, and deliberate combinations of these. In addition to statistical methods, Principal Component Analysis (PCA) will contribute to the feature base. Classification systems will combine and filter multiple features with thresholds and morphological operations to designate a signal (pixel intensity over time) as heat plume or not.

Since one goal is to keep cost down, the methods herein will use only one band. IR cameras used by fire fighters (for example, the FX 160 by Infrared Cameras Inc.) detect in long- 
wave because it cuts through smoke. Therefore the only logical band to use is the CMWIR, which does show a stronger smoke signal. Furthermore, the LWIR is far more prone to light blooming, which drastically obscures the signal of the heat plume. Therefore, the only useful band available is CMWIR, and all processes in this thesis will use CMWIR.

Figure 1 shows a typical frame with active fire and heat plume in it. The red rectangle defines the area of interest that the majority of the methods will operate on. Focusing on a small region reduces computation time and storage requirements, facilitating faster prototyping. The area of interest includes the fire and heat area, and challenging areas: foliage near and long ranges, people walking, and cars driving by.

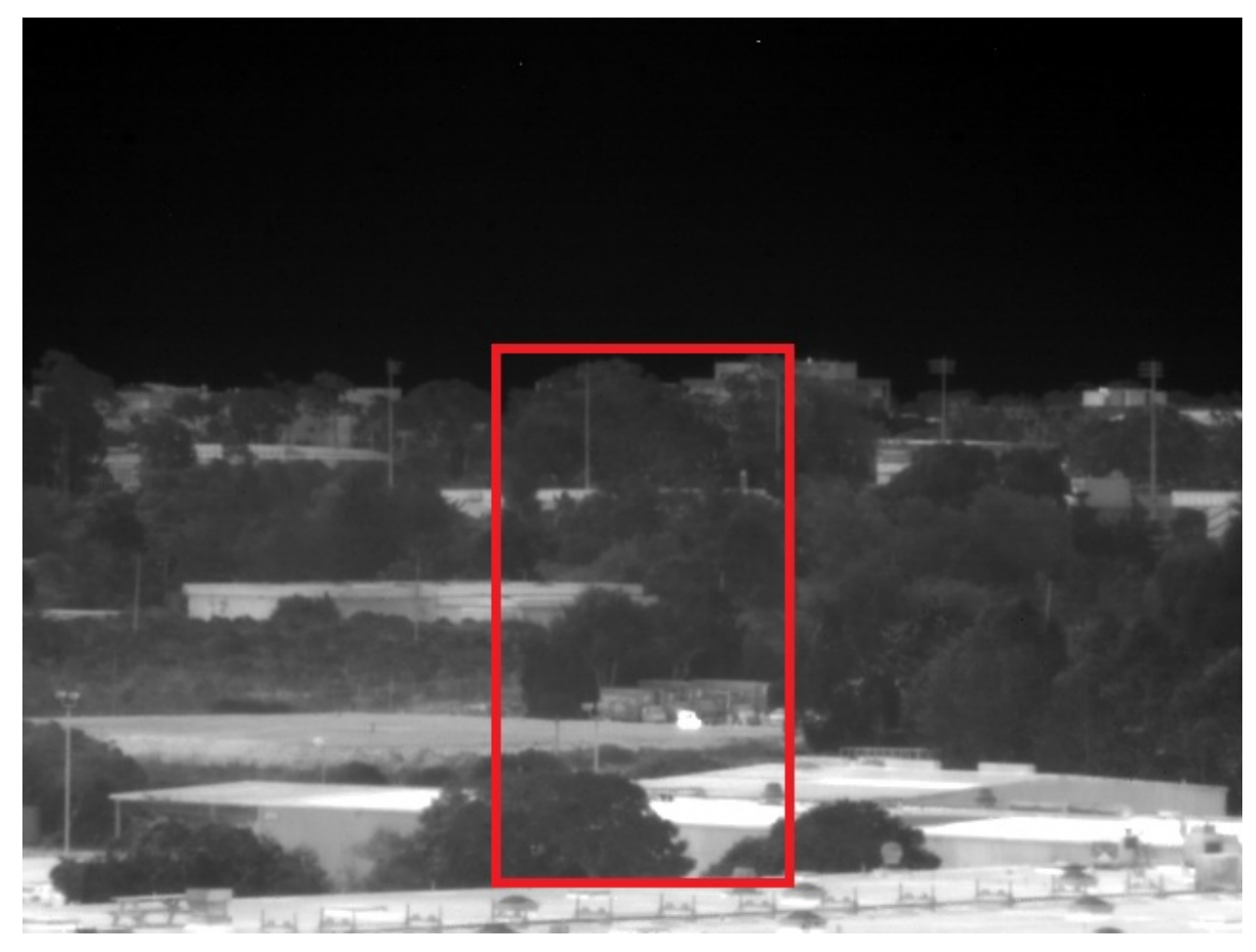

Figure 1 - Typical Frame in Scene Showing Region of Interest, Containing Active Fire (pine_cones channel, frame 2000) 


\subsection{Document Organization and Notes to the Reader}

Chapter 2 will discuss data acquisition, which describes the data collection mission and the video stabilization. Chapter 3 will discuss the features considered here-in, including their derivation and an analysis of merit. In some cases, experiments were performed that will be presented. Chapter 4 will introduce intensity maps that later algorithms will use. Chapter 5 will develop classifiers, which combine features into decisions on heat plume presence, using different methods. These methods will be defined and analyzed in a similar fashion as the features to study and improve their quality as fire alarms. The methods will also be developed and tuned in response to the analysis. Chapter 6 will conclude with a comparison and summary of the methods tested, suggest future avenues of inquiry, and offer lessons learned. Figure 2 shows a top-level block diagram showing the data flow throughout this thesis.

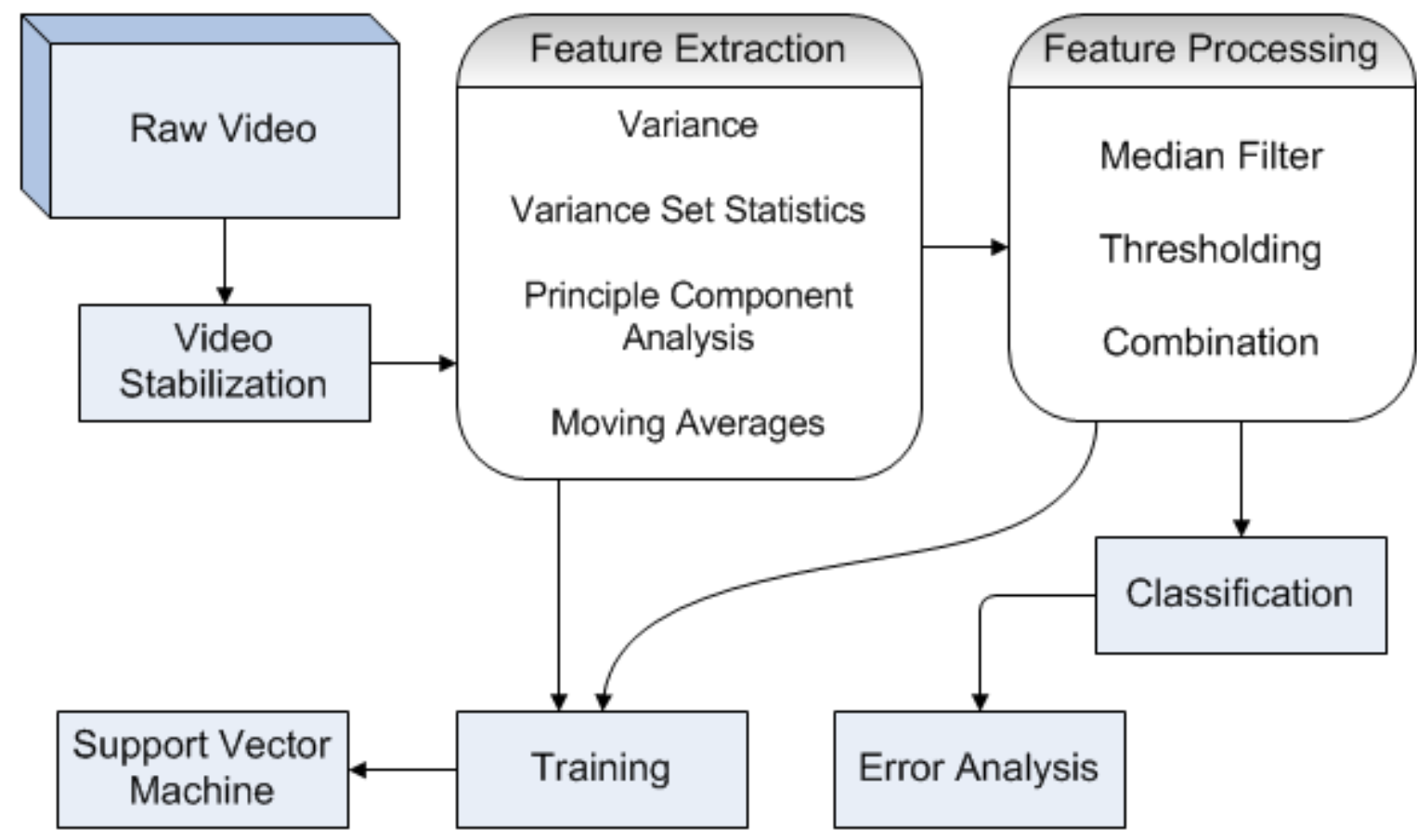

Figure 2 - Top-Level Block Diagram 


\subsubsection{Implementation Notes}

All primary research was performed in Matlab. Though this document focuses more on the theory, principles, thoughts, results, and observations, this text will refer to function names and code snippets to guide the reader who aspires to reproduce the work described herein. Where appropriate, the document will contain tables of functions with brief descriptions. The appendices contain the code that produced the results shown herein. The full code, including all referenced functions, should be attached to this document. The table of function descriptions will adhere to the format demonstrated in Table 1.

Table 1 - Generic Format for Function Description Tables within This Document

\begin{tabular}{|c|c|c|}
\hline \multirow[t]{2}{*}{ Function / Script Name } & \multicolumn{2}{|c|}{ Description Overview } \\
\hline & Function Input & Function Output \\
\hline script 1 & \multicolumn{2}{|c|}{$\begin{array}{l}\text { A script has no inputs nor outputs, only a sequence, or batch, of } \\
\text { commands, hence it will not have two descriptive columns }\end{array}$} \\
\hline script2 & \multicolumn{2}{|l|}{ This is a nondescript script. } \\
\hline functionRed & $\begin{array}{l}\text { A function may be described } \\
\text { in terms of its inputs and } \\
\text { outputs. }\end{array}$ & $\begin{array}{l}\text { A code file may be identified as a } \\
\text { function by having these two } \\
\text { columns. }\end{array}$ \\
\hline functionBlue & $\begin{array}{l}\text { Functions may have multiple } \\
\text { inputs and outputs. }\end{array}$ & $\begin{array}{l}\text { The output description makes the } \\
\text { function's purpose evident. }\end{array}$ \\
\hline anotherScript & \multicolumn{2}{|c|}{ Some deem this script redundant, but it is well commented. } \\
\hline
\end{tabular}


All raw and derived data will be stored in an organized fashion by band, then channel, then by category, as in the following example:

- Band: CMWIR

○ Channel: oats1

- Category: features I classification I training

- SMA_seq30

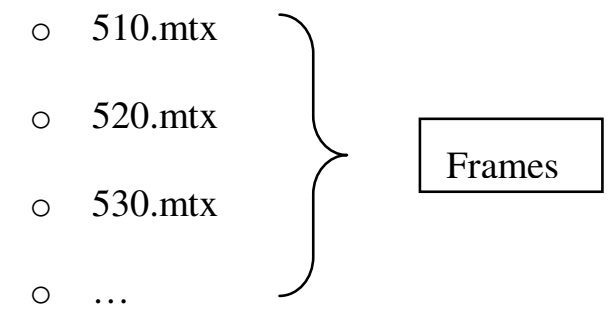

The raw frames are stored in .mtx format, which contains dimension and data type information, making it hassle-free to read from and write to.

The deepest folder (SMA_seq30, a feature) contains a sequence of raw frames. This method of storing data ensures scalability, and facilitates intuitive manipulation of more than 100,000 files, in more than 120 folders.

\subsubsection{Video Demonstration}

This thesis will be archived with accompanying video demonstrations associated with the classifiers in Chapter 5. If the demo video was the source of a figure, then the video name will be referenced in the figure caption.

\subsubsection{Clip Art}

All images and clip art in this document are either original or taken from public domain sources. 


\section{DATA ACQUISITION}

Raytheon Vision Systems in Goleta, California sponsors Cal Poly's efforts into forest fire detection, and one way they contribute is by offering the use of their site and equipment. All data used in this thesis was collected from a mission to Goleta. The raw data was then pre-processed in preparation for use in the algorithms developed in Chapters 0 and 4.

\subsection{The Goleta Mission}

On June 18, 2011, a team embarked on a data collection mission to Goleta, California, to Raytheon's Remote Vision Systems site. For the purpose of the mission, the RVS site consisted of a control room, a tower with a set of cameras mounted on top just outside the control room, and a parking lot with a Santa Maria style barbecue pit. The camera tower elevated the cameras to approximately $20 \mathrm{~m}$ high, and stood about $800 \mathrm{~m}$ distant from the barbecue pit. Figure 3 shows the location, courtesy of Google Maps.

\subsubsection{Setup}

At the control room, cables relayed the data from the cameras on the tower to computers, where the team used software to capture and record the data to hard drives. Three cameras took data in five bands. One camera took RGB video; one un-cooled camera took long-wave infrared (UCLWIR), and one camera with an in-built cooling system took both long-wave and mid-wave infrared video (CLWIR, CMWIR, respectively). This thesis doesn't consider visible nor shortwave, so those will not be mentioned further. Table below shows individual camera specifications, and Table 3 shows common specifications. 


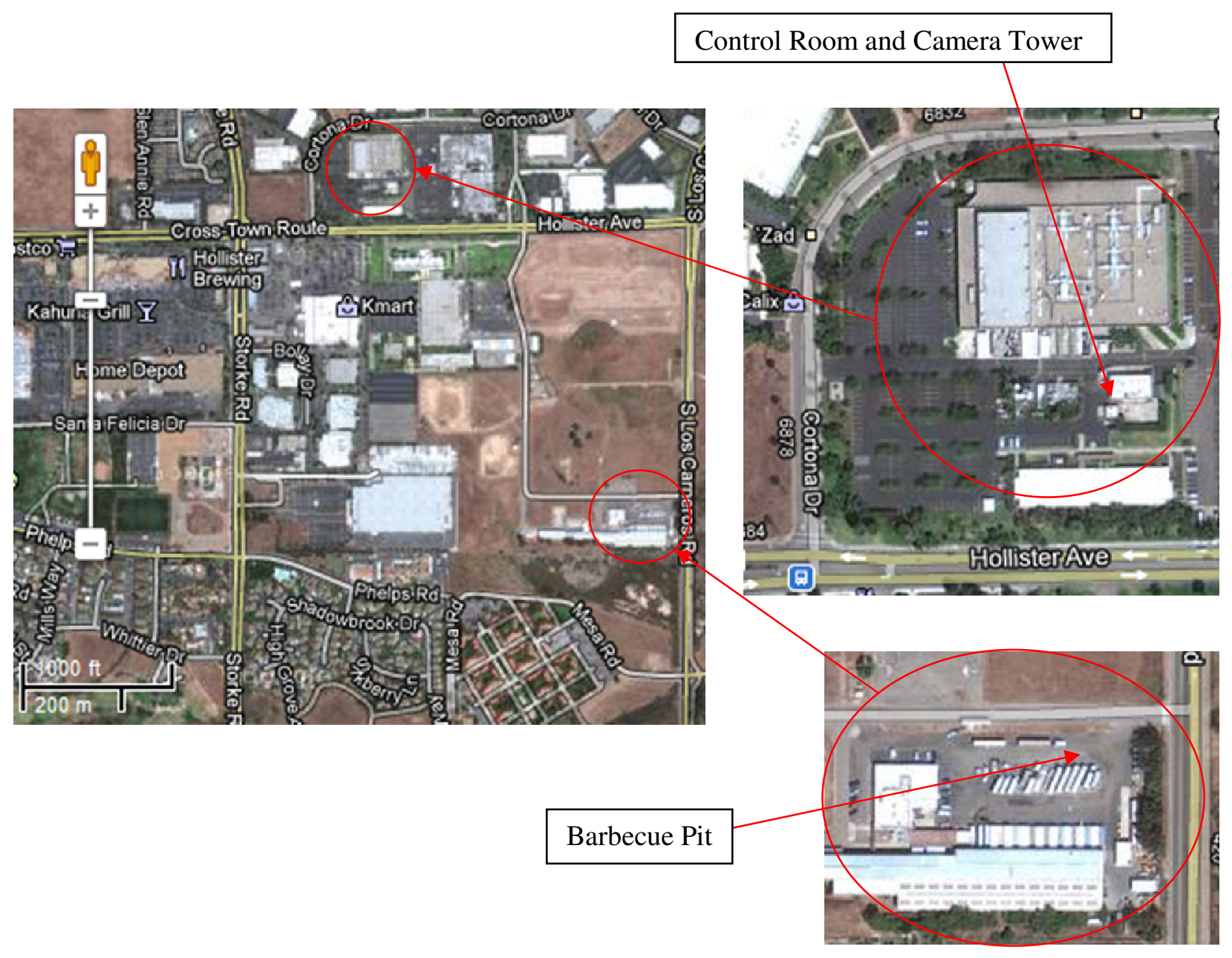

Figure 3 - Data Collection Setup Locations-of-Interest

Table 2 - Individual Camera Specifications

\begin{tabular}{llll}
\hline & Focal Length & Aperture & Pixel size \\
\hline Uncooled LWIR & $75 \mathrm{~mm}$ & F1 & $25 \mathrm{um}$ \\
Cooled LWIR & $100 \mathrm{~mm}$ & F2 & $20 \mu \mathrm{m}$ \\
Cooled MWIR & $100 \mathrm{~mm}$ & F3 & $20 \mu \mathrm{m}$ \\
\hline
\end{tabular}

Table 3 - Common Camera Specifications

\begin{tabular}{ccc}
\hline Resolution & Nominal Sample Rate & Format \\
\hline $\mathbf{6 4 0} \times 480$ & $30 \mathrm{fps}$ & 14-bit little-endian raw \\
\hline
\end{tabular}


At the barbecue pit, the team had brought several varieties of fuel, for the purpose of collecting a wider range of data. Perhaps research on the video can discriminate between different fuels, or perhaps one fuel would give a stronger signal. The following fuels were gathered and burned in this mission:

- Charcoal

- Grass and wild oats

- Pine needles

- Pine cones

- Palm leaves
- Thistles

- Wet leaves

- Damp and dry leaves

- Wild rosemary

- Apple wood

\subsubsection{Procedure}

One person at each site had a dedicated communications role by cell phone, to facilitate coordination of efforts. The communicator also served as the dedicated note-taker on the sequence and timing of events. Since the mission was expected to last a few hours, it was divided into sections in which one fuel was burned, and the video take saved in separate files. One operator per computer remotely controlled the cameras and saved the data according to an established naming convention.

The fuel burn order was planned in advance. For each take section, the control room communicator would log the time and fuel type, direct the computer operators to begin recording, then direct the barbecue pit team to add the fuel to the pit. Throughout the burn, both the control room note-taker and the barbecue site note-taker recorded visual observations of the burn from their perspective. A typical take lasted approximately 10 minutes.

\subsection{Raw Data}

The data format is 14-bit little-endian, saved as the most significant bits of 16-bit words, where the unused 2 LSBs were assigned to zero. The image pixels are stored in $x-y$ order, one 
pixel after the other in a left-to-right row, then one column after another in top-to-bottom columns, then one frame after another through time. Each video is saved to disk as a 1dimensional bit stream in this fashion. Figure 4 illustrates the data word format.

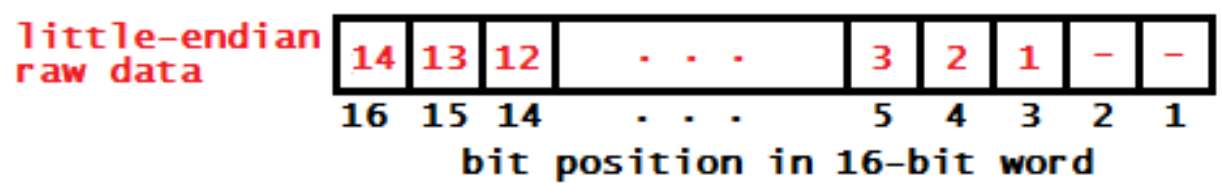

Figure 4 - 14-Bit Little-Endian Raw Data Saved in 16-Bit Word

The cooled camera that captured CMWIR and CLWIR data passed data to a buffer that concatenated frames in the x-dimension. Each channel updated at $30 \mathrm{fps}$, staggered with each other, so that the buffer updated at $60 \mathrm{~Hz}$. At each update, the buffer sent its data back to the control room computers via Ethernet (See Figure 5). The computers received and recorded the video frames at the same rate the buffer sent them. Therefore, each cooled infrared video file contains redundant data. The UCLWIR data path had no such complications as the single camera sent its data stream back to the control room, which saved the single stream to its raw data file.

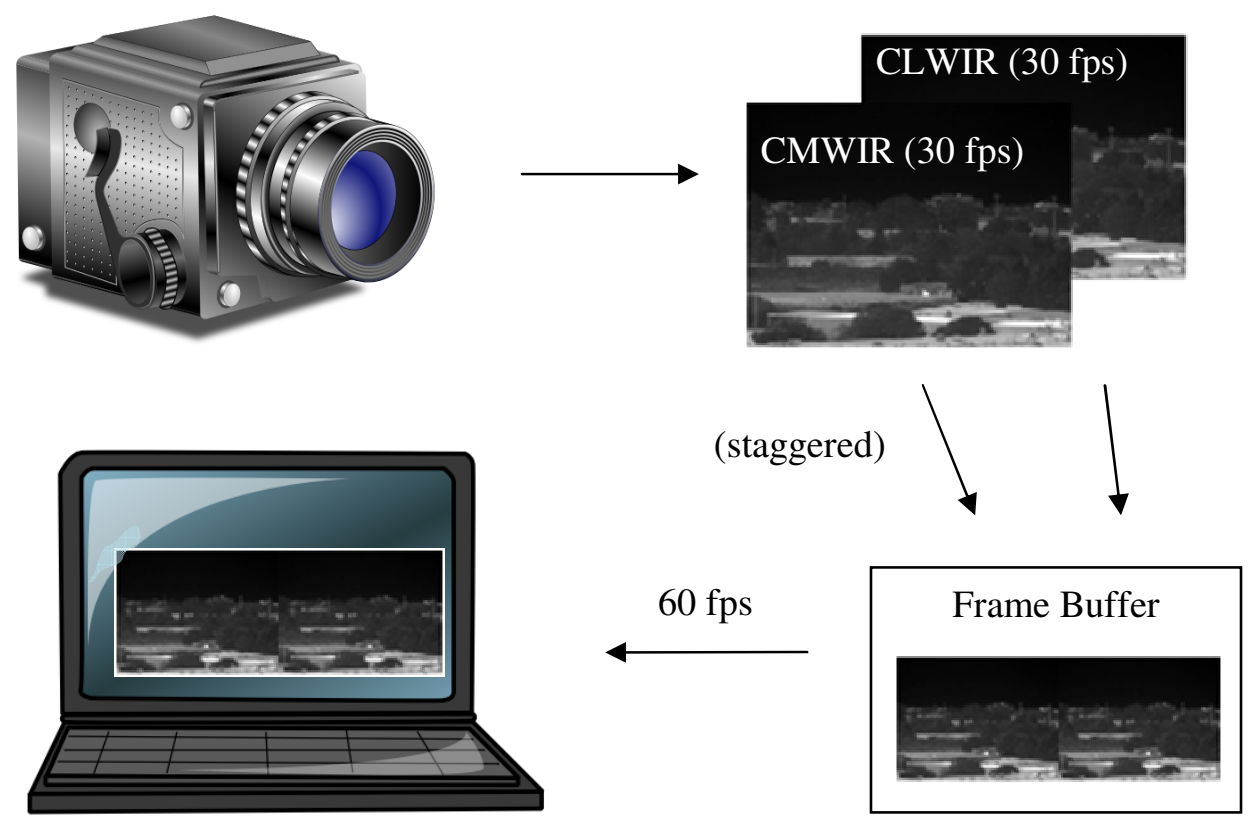


Table 4 shows a subset of the raw data files that this thesis will use. The cooled data file names have the convention:

$$
\text { (date)_(time)_LW-MW_(fuel name).raw }
$$

The un-cooled (UCLWIR) file names have the convention:

$$
\text { (date)_(time)_(fuel name).raw }
$$

Note by inspection of the file names that both long-wave and mid-wave data are stored together. Each file contains 8000 frames, so the file size of the cooled LW-MW file is:

$$
\left(640 \cdot 480 \frac{\text { pixel }}{\text { frame }}\right) \cdot\left(2 \frac{\text { bytes }}{\text { pixel }}\right) \cdot\left(2 \frac{\text { frame }}{\text { file }}\right) \cdot\left(2 \frac{\text { written frame }}{\text { captured frame }}\right)=19,660,800,000 \text { bytes }
$$

The file size of the UCLWIR is:

$$
\left(640 \cdot 480 \frac{\text { pixel }}{\text { frame }}\right) \cdot\left(2 \frac{\text { bytes }}{\text { pixel }}\right) \cdot\left(1 \frac{\text { frame }}{\text { file }}\right) \cdot\left(1 \frac{\text { written frame }}{\text { captured frame }}\right)=4,915,200,000 \text { bytes }
$$

Table 4 - Raw Data Files Used

\begin{tabular}{lll}
\hline Raw Data File & Size (bytes) & Source \\
\hline 2011-06-18_102454_LW-MW-14bit_oats1.raw & $19,660,800,000$ & Cooled \\
\hline 2011-06-18_102646_oats1.raw & $4,915,200,000$ & Uncooled \\
2011-06-18_105906_LW-MW-14bit_pine_cones.raw & $19,660,800,000$ & Cooled \\
2011-06-18_110100_pine_cones.raw & $4,915,200,000$ & Uncooled \\
\hline
\end{tabular}

Note: For the remainder of this paper, if a video channel is referenced but not specified as stabilized or pre-stabilized, assume that it is stabilized (Explained in 2.4). 


\subsection{Data Extraction and Manipulation}

The large raw data files (Table 4) were separated into 8000 files containing one raw frame each (Figure 6). This process performed the following operations:

- The 16-bit words were right shifted by 2 bits to place the LSB of the 14-bit information word into the LSB position of the 16-bit recorded word. This moves the 2-bit zero padding to the 2 MSBs instead of the 2 LSBs.

- Convert (x, y) order into (r, c) order (explained below).

- For the concatenated long-wave and mid-wave file,

- Separate the two channels into different files

- Discard every other frame from each channel in a staggered pattern (fixCooledIndicex.m).

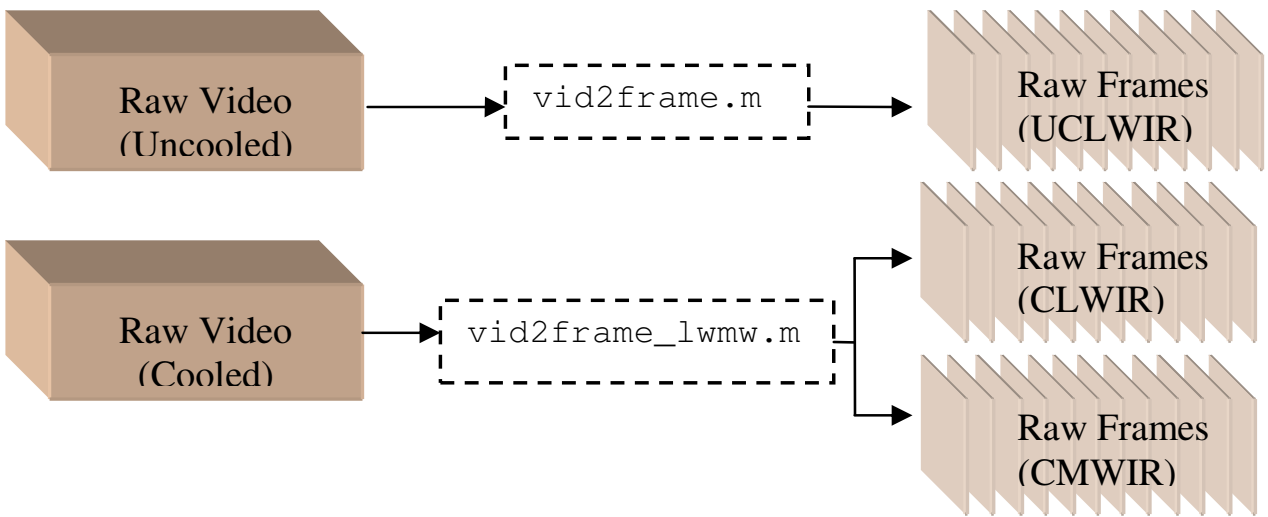

Figure 6 - Raw Data File Frame-Wise Separated

The cameras recorded raw frames in $(\mathrm{x}, \mathrm{y})$ order into the data stream (left-to-right, top-tobottom), but Matlab interprets the stream in (r, c) order (top-to-bottom, left-to-right). To simplify operations throughout the project, the $(\mathrm{x}, \mathrm{y})$-ordered frames from the raw video were converted to $(\mathrm{r}, \mathrm{c})$-ordered frames before saving them to the raw frame files. The ordering matters because files are 1-dimensional data streams, so the reading function must know the order to correctly 
load that 1-dimensional data into 2-dimensional imagery. Figure 7 shows a diagram of the data order from the camera to the raw video file to the raw frame files.

\begin{tabular}{|c|}
\hline row1 \\
\hline row2 \\
\hline row3 \\
\hline$\ldots$ \\
\hline
\end{tabular}

\begin{tabular}{|c|c|c|c|}
\hline $\mathrm{c}$ & $\mathrm{c}$ & $\mathrm{c}$ & \\
$\mathrm{o}$ & $\mathrm{o}$ & $\mathrm{0}$ & \\
$\mathrm{I}$ & $\mathrm{I}$ & $\mathrm{I}$ & $\ldots$ \\
1 & 2 & 3 & \\
\hline
\end{tabular}

(a) Camera writes data stream to file in $(x, y)$ order

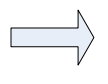

\begin{tabular}{|l|l|l|}
\hline row1 & row2 & $\ldots$ \\
\hline
\end{tabular}

(b) Matlab interprets data stream in ( $r, c)$ order, so that the image rows are oriented as columns

(d) Matlab may now read and write in $(r, c)$ order (c) Transpose

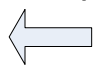

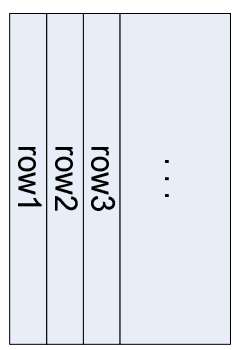

col1 col2

Figure 7 - Frames Converted from (x, y) Order to (r, c) Order

In Figure 7 step (b), the function (vid2frame.m or vid2frame_lwmw.m from Figure 6) reads the raw video file by columns, yet the camera wrote the data by rows, hence the image becomes transposed: the top row becomes the left column. The figure illustrates this transposition by showing "row1", "row2", etc., rotated. After a corrective transpose in step (c), the image would display in correct orientation. The columns, designated "col1", "col2", etc., are vertically oriented, again showing that the image would display correctly. Step (d) represents the reversible process of writing to and reading from a file in (r, c) order: top-to-bottom, left-to-right.

Now the raw video information is separated into frames, allowing easy access to any frame. Several functions were developed to facilitate data reading and writing, and visualization. 
Table 5 - Data Acquisition and Manipulation Function Descriptions

\begin{tabular}{|c|c|c|}
\hline \multirow[t]{2}{*}{ Function / Script Name } & \multicolumn{2}{|c|}{ Description Overview } \\
\hline & Function Input & Function Output \\
\hline $\begin{array}{l}\text { vid2frame }^{1}, \\
\text { vid2frame_lwmw }\end{array}$ & \multicolumn{2}{|c|}{$\begin{array}{l}\text { Script that converts raw video files into separate files containing } \\
\text { one frame each, and saves them in hierarchical folders. }\end{array}$} \\
\hline fixCooledIndices & \multicolumn{2}{|c|}{$\begin{array}{l}\text { Corrects redundant frames generated by vid2frame_lwmw. } \\
\text { Deletes even frame files, then renames odd frame files to be } \\
\text { contiguous. }\end{array}$} \\
\hline readframes $^{2}$ & $\begin{array}{l}\text { Location of raw video data } \\
\text { (already separated into one } \\
\text { frame per file), frame } \\
\text { dimension, desired frame } \\
\text { indices to read. }\end{array}$ & $\begin{array}{l}\text { Matrix of frames, matrix of } \\
\text { frame indices. }\end{array}$ \\
\hline slideshow & \multicolumn{2}{|c|}{$\begin{array}{l}\text { Calls readframes and displays the resulting video matrix in a } \\
\text { figure, displaying frames at an adjustable fixed rate, or advanced } \\
\text { by keystrokes, to allow examination of the video data. }\end{array}$} \\
\hline raw2bmp & $\begin{array}{l}\text { Path of raw input image, and } \\
\text { path to bmp output image. }\end{array}$ & $\begin{array}{l}\text { Imports specified raw image } \\
\text { and saves it as bmp. No } \\
\text { function output. }\end{array}$ \\
\hline saveMtx & $\begin{array}{l}\text { A matrix, save path, data type } \\
\text { (optional). }\end{array}$ & $\begin{array}{l}\text { No output. Writes the matrix } \\
\text { to file, preserving } \\
\text { dimensionality and type. }\end{array}$ \\
\hline loadMtx & $\begin{array}{l}\text { Path to a file in a format } \\
\text { consistent with saveMtx, data } \\
\text { type (optional). }\end{array}$ & $\begin{array}{l}\text { Returns the matrix in the same } \\
\text { dimensions it was written as. }\end{array}$ \\
\hline mtx2vid & Matrix of frames, save path. & $\begin{array}{l}\text { No output. Generates an AVI } \\
\text { video of the input frames at the } \\
\text { specified save path. }\end{array}$ \\
\hline
\end{tabular}

\subsection{Digital Video Stabilization by Point Feature Matching}

When viewed with the tools established in Section 2.3, it became apparent that the video contains motion jitter. The tower, upon which the cameras were fixed, moved slightly as the wind stimulated its high-frequency vibration modes, introducing the jitter. Motion jitter corrupts the temporal signature of each pixel, making the temporal signature of the heat plume more difficult to identify, warranting a video stabilization operation. 
Feature matching methods commonly consist of feature registration and matching between frames [8]. Point Feature Matching (PFM) stabilization spatially aligns a frame with another frame by registering and comparing salient points (e.g. corner pixels) between the frames [7]. Since the camera does not move much, all frames appear very similar to the first frame, used as a stable reference, so corner detection produces comparable sets of pixels. The Feature Accelerated Segment Test (FAST) criterion is used to identify corner pixels in both the reference frame and the test frame [10]. Correspondence between reference frame and test frame corner pixel pairs is assigned by choosing the least sum of square differences (SSD) between the 9-by-9 neighborhoods centered about each corner pair. The Random Sampling and Consensus (RANSAC) method throws out the correspondence outliers by assuming a subset of correspondences as inliers, computing a linear geometric transform that fits those inliers, then including all remaining correspondences that closely agree with the transform, as inliers. The linear geometric transform is re-computed for this expanded set of inliers, then the transform is evaluated on how well the transformed test frame matches the reference frame. RANSAC is done 10 times with a different random subset of initial correspondences, and the best fit transform is used to transform the test frame to the reference frame [11], vastly reducing the jitter. To ensure a stable image, every frame is stabilized against the first frame of the video, else accumulated error in the transform causes the view to migrate.

The PFM process is as follows.

1. Collect corner points from a pair of frames using the FAST (Features from Accelerated Segment Test) algorithm. See Figure 8. 


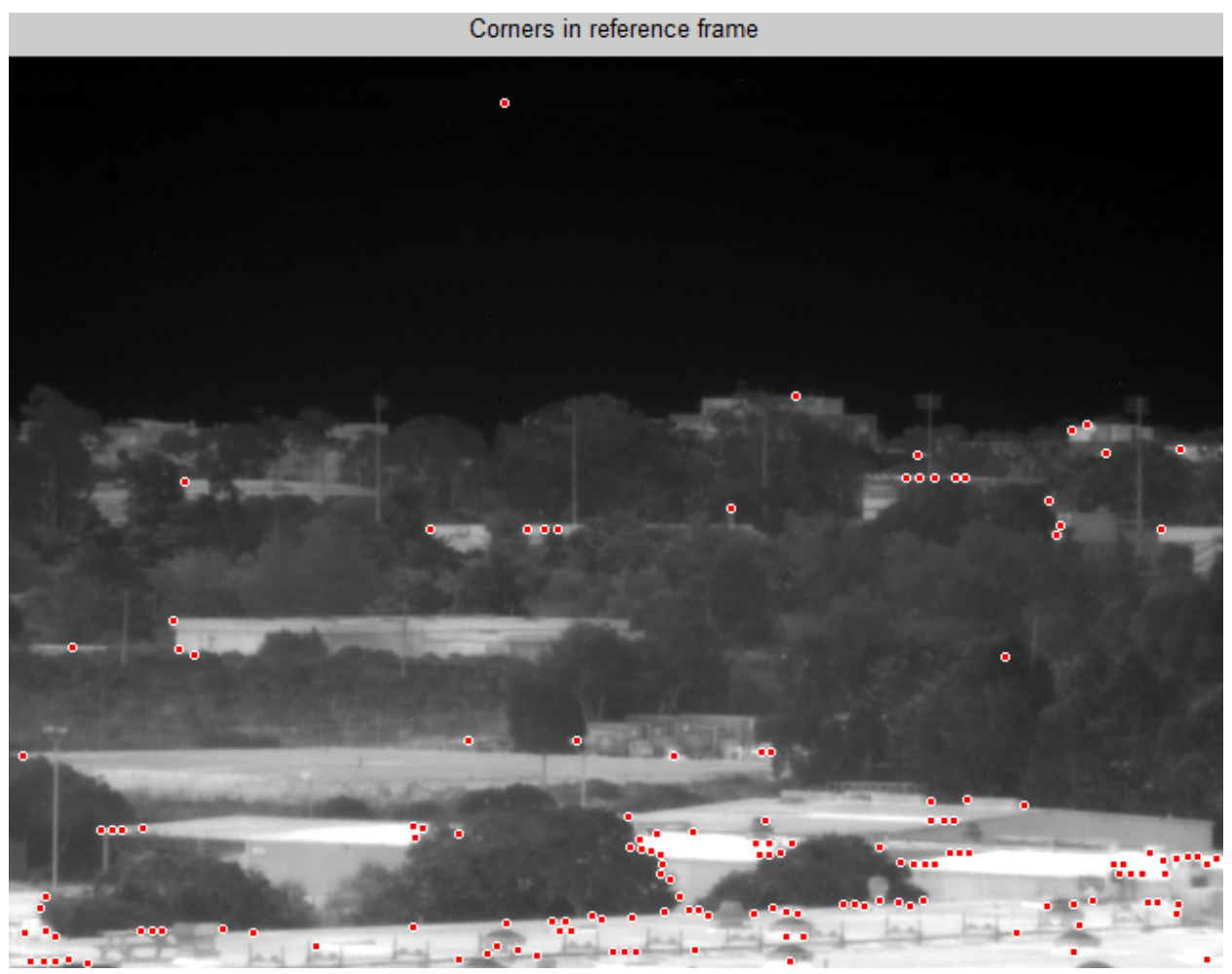

(a) Reference Frame

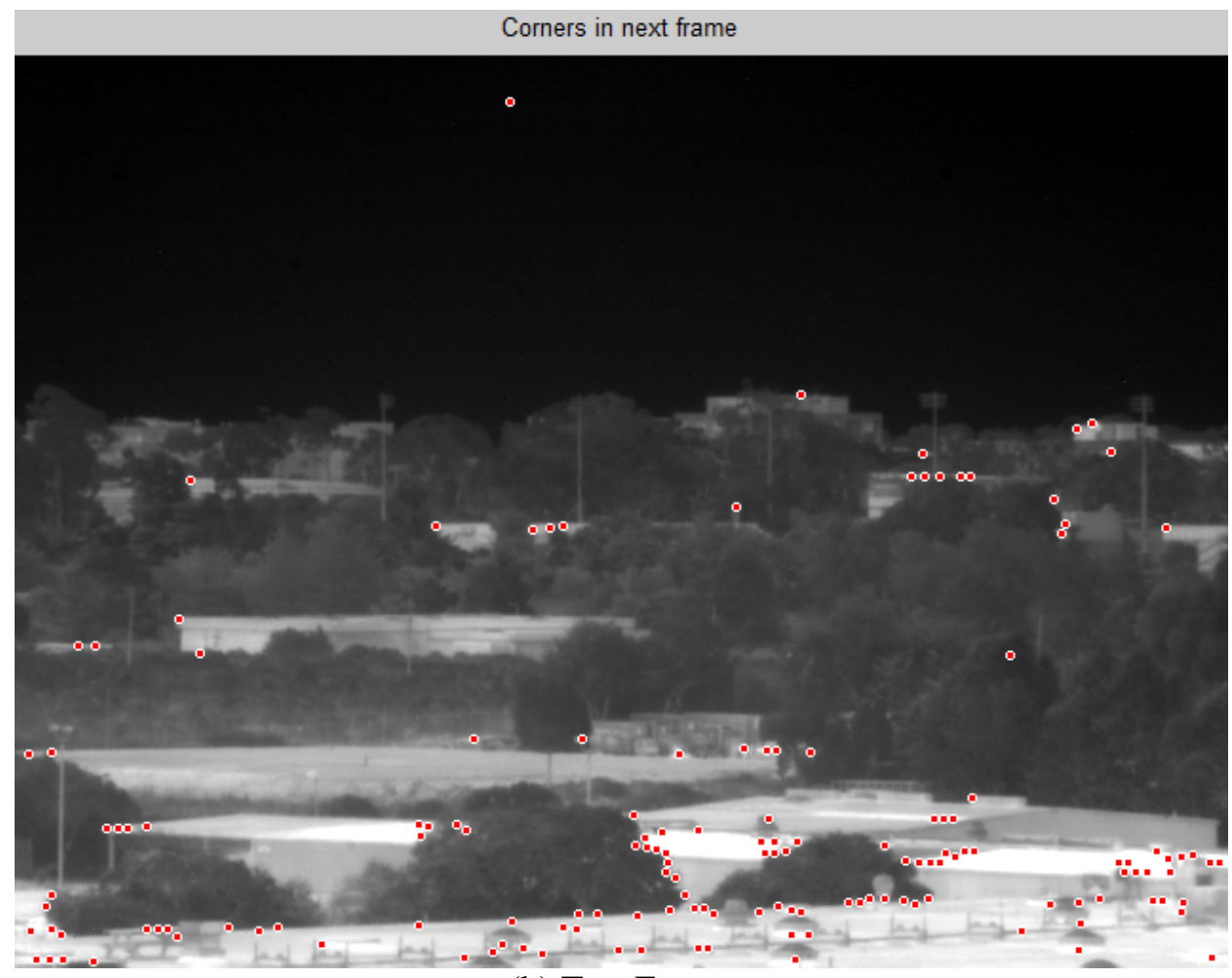

(b) Test Frame

Figure 8 - Corner Points Detected By FAST Algorithm 
2. Identify correspondences between corner points. See Figure 9. The yellow lines mark the assigned correspondences based on the minimum SSD. In this case, every visible line marks an incorrect correspondence, but all or most correct pairs correspond as well, and are marked with a 1-pixel yellow line.

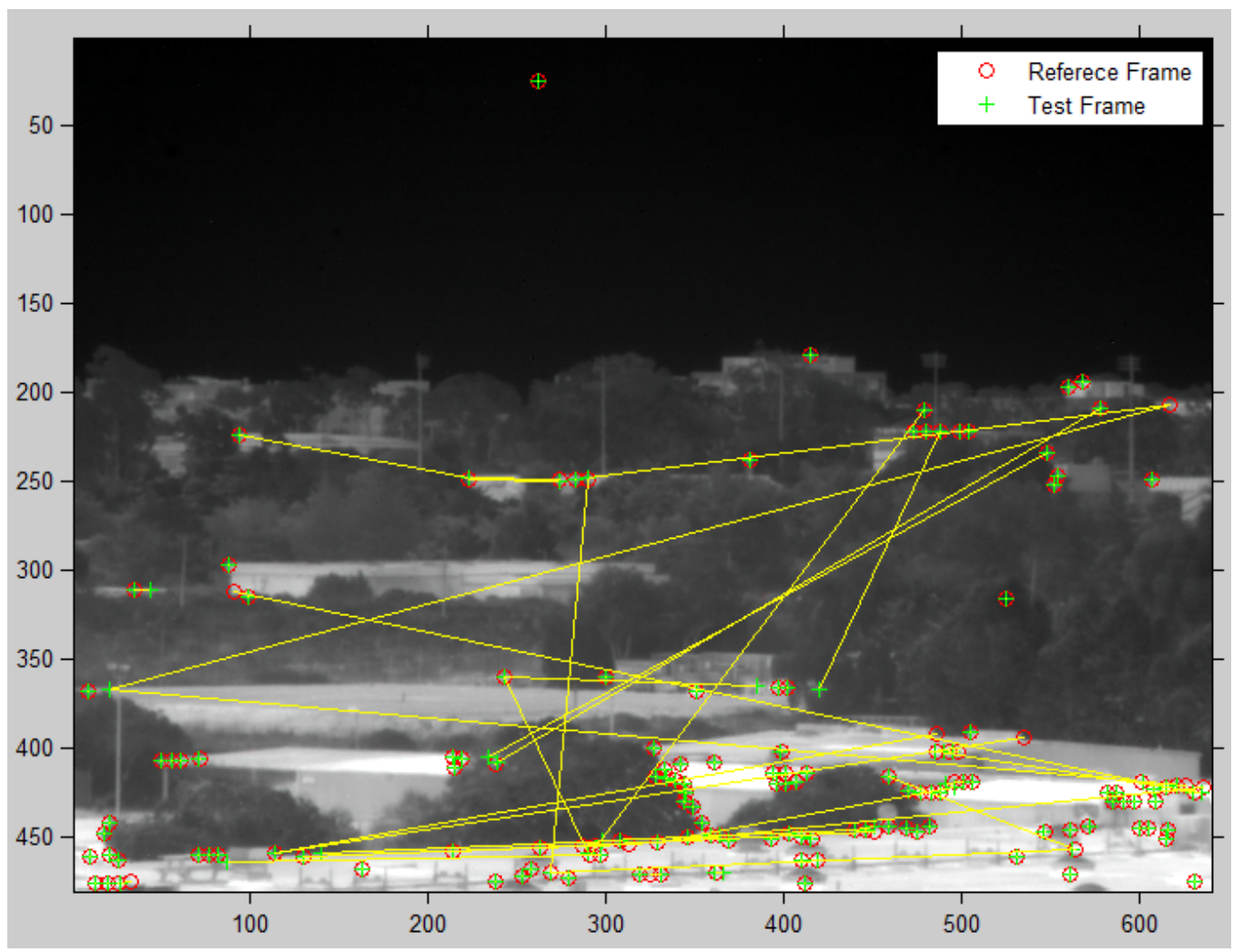

Figure 9 - Correspondences Between Corner Points

3. Use RANSAC method to eliminate correspondence outliers and select a well-fitting transform. Figure 10 shows the result with transform applied. Note the absence of outliers and the well-overlapping edge pixels between reference and test frames. 


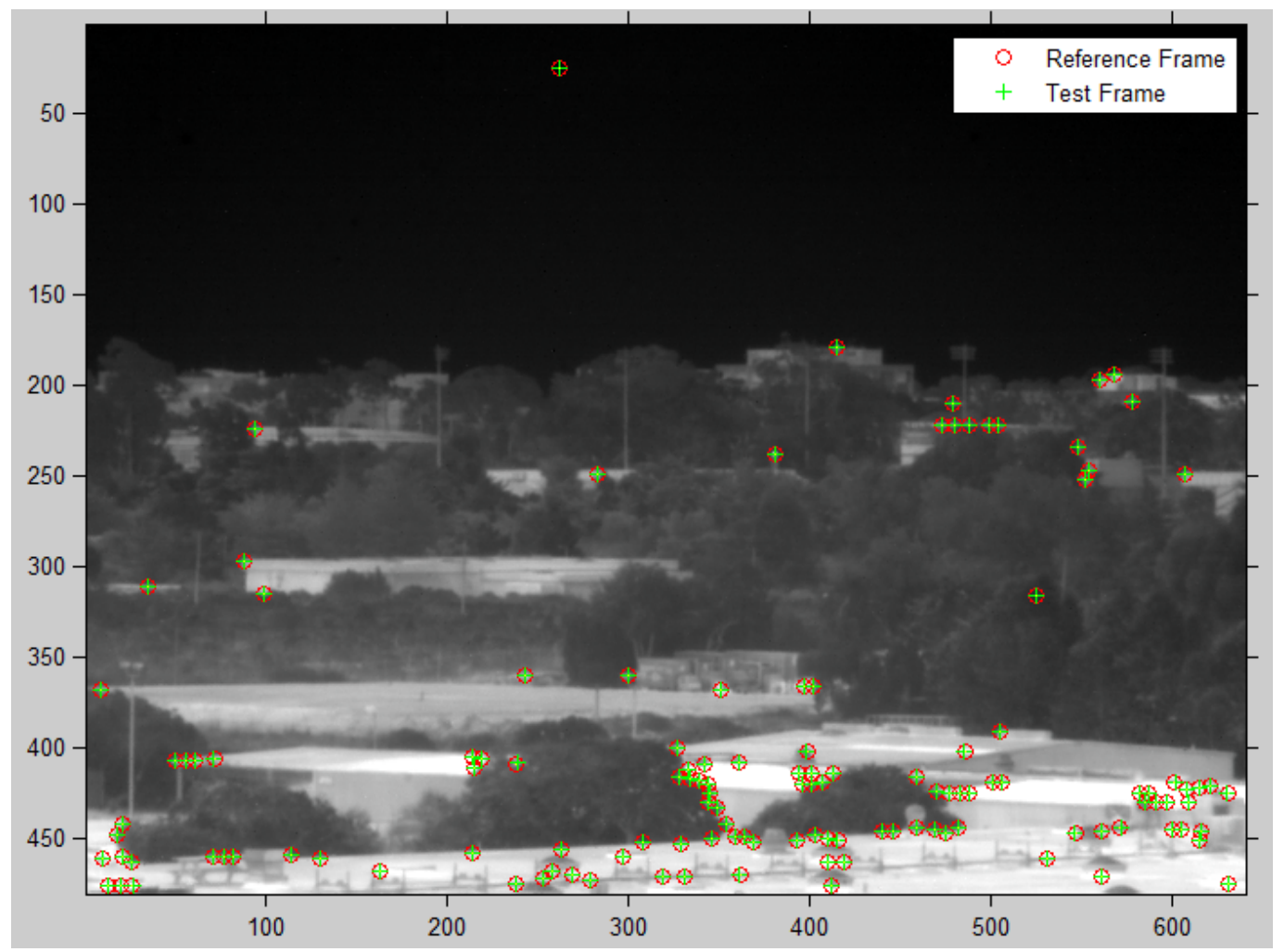

Figure 10 - Inlier Matches From 10 RANSAC Trials

In this manner, all frames of a raw video align to the first frame. The functions in Table 6 perform the stabilization operation.

Table 6 - Video Stabilization Function Descriptions

\begin{tabular}{lll}
\hline \multirow{2}{*}{ Function / Script Name } & \multicolumn{1}{c}{ Description Overview } \\
\cline { 2 - 3 } stabilizeVidPF & \multicolumn{1}{c}{ Function Input } & \multicolumn{1}{c}{ Function Output } \\
\hline stabilizeVidPFraw & $\begin{array}{l}\text { A frame matrix, ostensibly with } \\
\text { motion jitter. }\end{array}$ & $\begin{array}{l}\text { A frame matrix of equal size, } \\
\text { motion stabilized. }\end{array}$ \\
& $\begin{array}{l}\text { Script that performs video stabilization upon a specified channel } \\
\text { of individual raw frames. Specify with sourceName variable. } \\
\text { Writes individual raw frames to a parallel folder named as the } \\
\text { source folder + "_PFstab". }\end{array}$ \\
\hline
\end{tabular}

To measure the effectiveness of the video stabilization, consider the variance images (described in section 3.1.1) shown in Figure 11. 


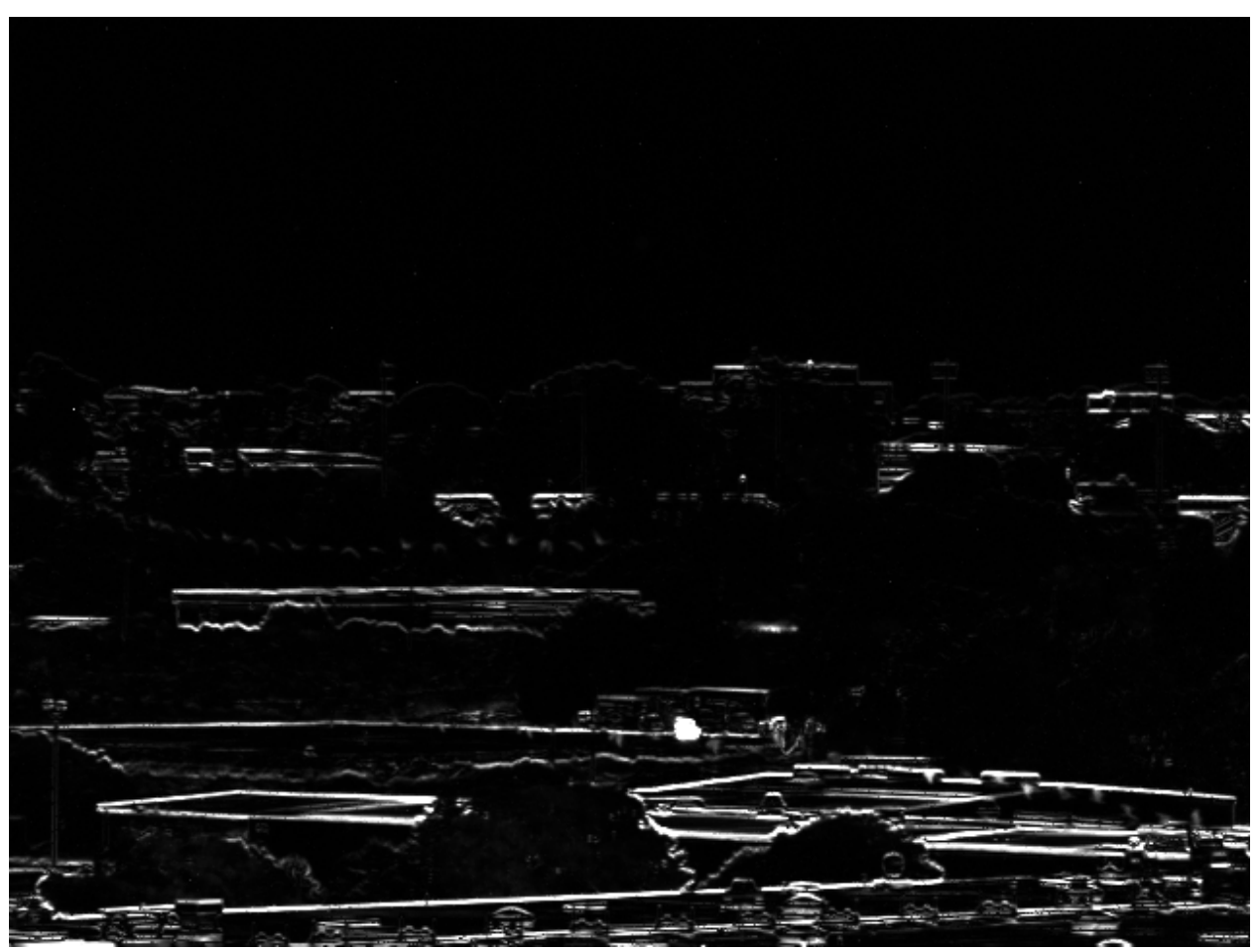

(a) Before Stabilization

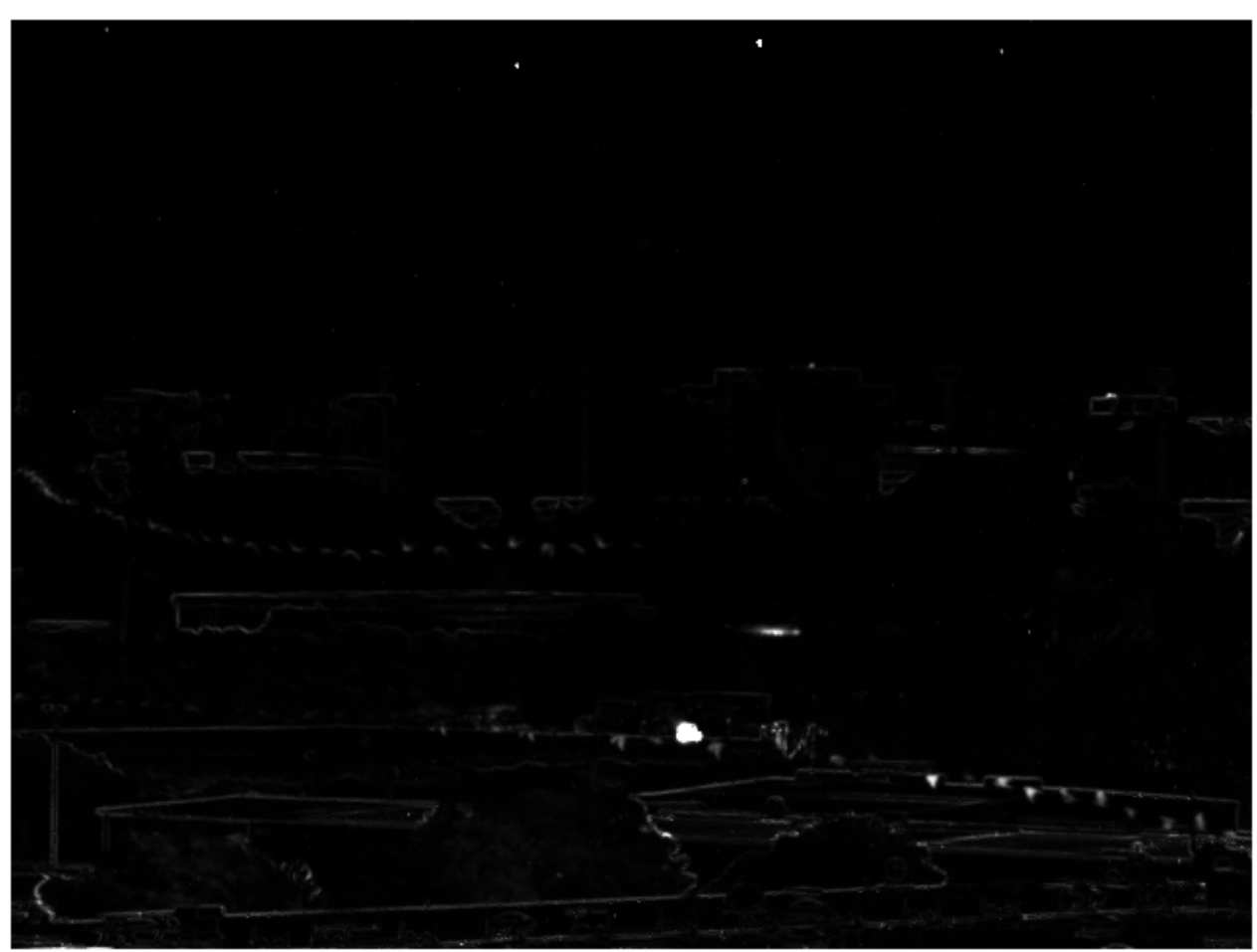

(b) After Stabilization

Figure 11 - Variance Frames Before and After Stabilization

(pine_cones channel, frames 2000 through 2200) 
The first shows variance before stabilization, and the second shows variance after stabilization, for the same time range (frame 2000 to 2200 , step 2), and the same intensity range. High variance magnitudes associated with light-dark interfaces significantly reduce without the motion jitter, while the legitimate movement in the scene due to the heat appears uncompromised. Jitter-related noise corrupts every signal in the scene, and more so in areas of high contrast. To demonstrate, consider a perfectly stationary high-intensity pixel adjacent to a perfectly stationary low-intensity pixel. The variance of both pixels over time evaluates to zero. Now add motion jitter with magnitude of 1 pixel, such that the dark and light pixels periodically invade each others' space for short durations. The variance for both pixels will artificially evaluate high. If these two pixels were the same intensity, the distorted variance would evaluate to zero, equivalent to the true variance. Infer from this thought experiment that movement metrics suffer distortion in proportion to the contrast within a locality defined by the magnitude of the jitter, a conclusion supported by Figure 11. Some jitter remains, presumably due to spatial resolution constraints of the cameras.

Note: For the remainder of this paper, if a video channel is not specified as stabilized or prestabilized, assume that it is stabilized.

\subsection{Initial Data Examination}

Before delving into the features, and algorithms, let's visualize the data. Figure 12 shows a frame (2392) of the pine_cones video, before stabilization. 14-bit data can express a range of $\left[0,2^{14}-1\right]=[0,16383]$. This frame has a full-scale range (FSR) from [4801, 12215]. 


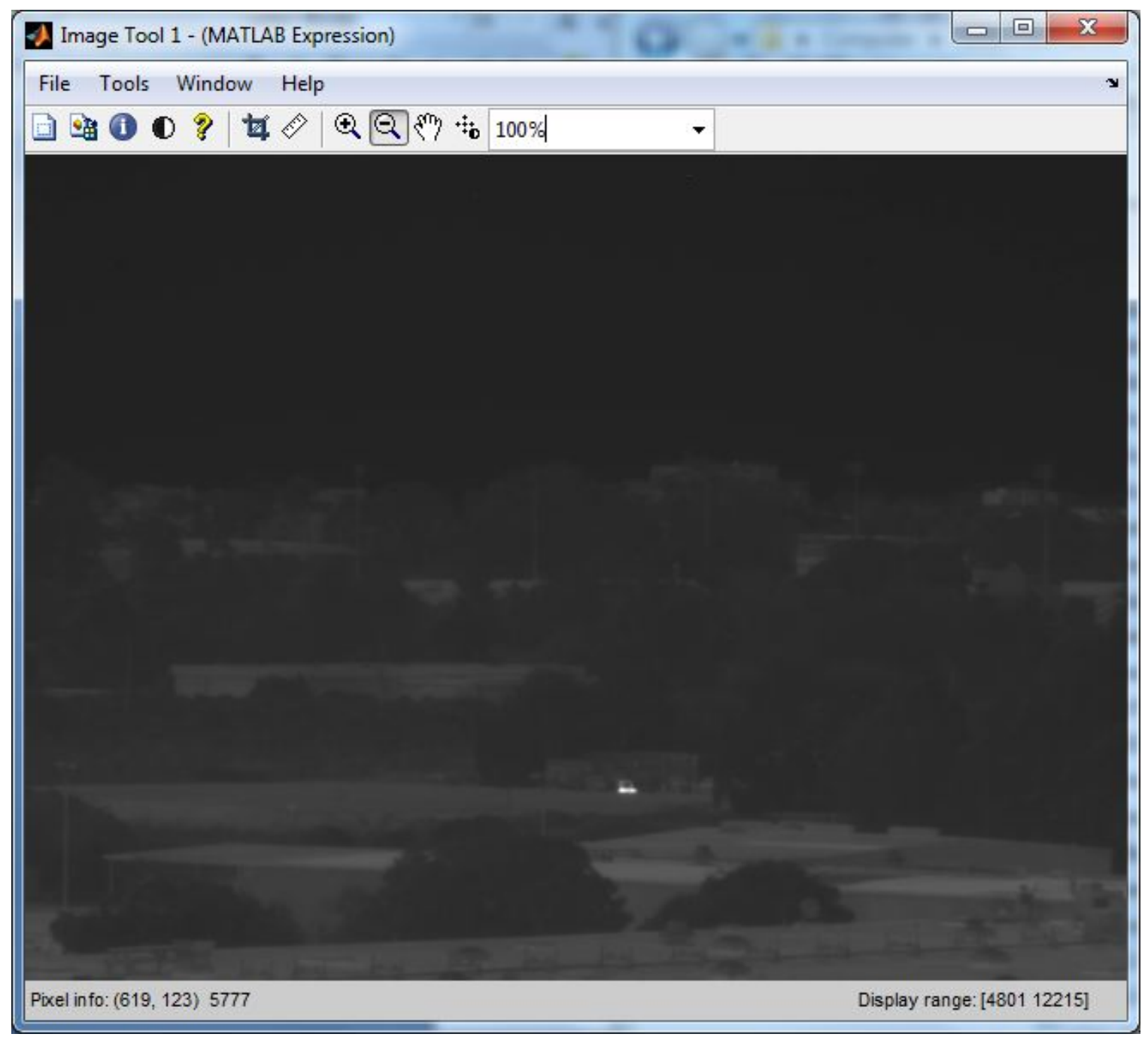

(a) Full-Scale Dynamic Range (4801 - 12215)

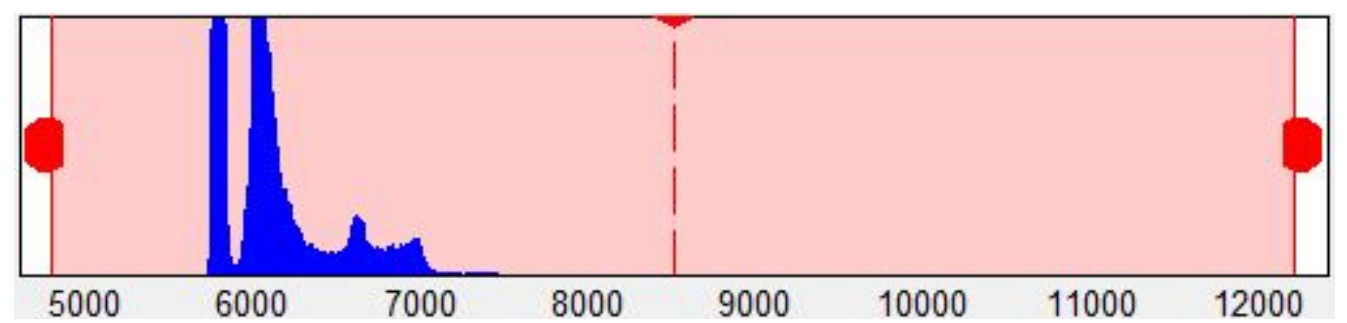

(b) Histogram

Figure 12 - Frame 2392 of pine_cones (not stabilized) 


\begin{tabular}{|c|c|c|c|c|c|c|c|c|c|c|c|}
\hline 6921 & 7249 & 7640 & 7602 & 7226 & 7230 & 7068 & 6976 & 6837 & 6501 & \multicolumn{2}{|c|}{ Barbecue har } \\
\hline 7015 & 7486 & 7943 & 7508 & 7155 & 7032 & 6913 & 7025 & 7079 & 6613 & 6393 & 6353 \\
\hline 7057 & 7679 & 8258 & 7538 & 7041 & 6949 & 6868 & 7196 & 7429 & 6776 & 6468 & 6386 \\
\hline 6919 & 7950 & 8955 & 8148 & 7628 & 7558 & 7398 & 7817 & 8112 & 7207 & 6715 & 6503 \\
\hline 7459 & 9589 & 11348 & 10747 & 10148 & 9755 & 9461 & 9951 & 10093 & 9039 & 8194 & 7103 \\
\hline 8041 & 10975 & 11959 & 12115 & 12019 & 11252 & 11149 & 10740 & 11803 & 11147 & 10138 & 7883 \\
\hline 7751 & 9105 & 10214 & 10322 & 10005 & 9304 & 9326 & 10058 & 10093 & 9768 & 8727 & 7617 \\
\hline 6978 & 7279 & 7500 & 7534 & 7508 & 7420 & 7454 & 7611 & 7639 & 7536 & 7384 & 6996 \\
\hline
\end{tabular}

Figure 13 - Close-Up of Barbecue Pit with Intensity Values Shown 


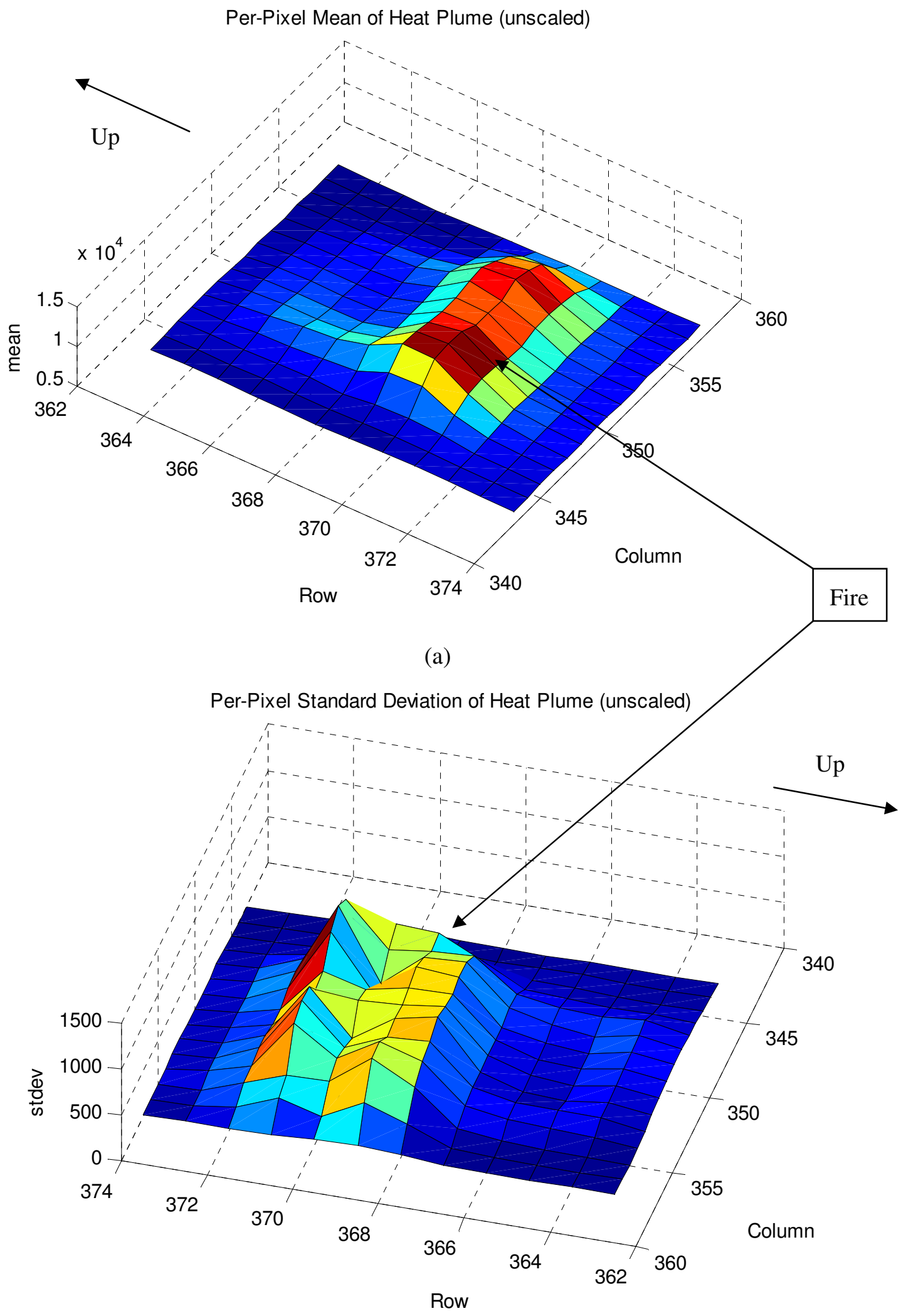

(b)

Figure 14 - Mean and Standard Deviation Per-Pixel of Barbecue Pit 
For the majority of this document, only a sub-range region of interest of the full frame will be operated on, to save memory requirements and computation time. The region of interest spans a wide range of distance from the camera, including the barbecue pit, foreground foliage, mid-ground foliage, distant foliage, a stationary uniform grassy field, and a stretch of road in view to catch traffic activity. See Figure 1.

\subsection{Challenges}

\subsubsection{Decaying Frame Rate}

The frame rate dropped as a recording progressed. The capture software reported the frame rate of the incoming video stream, but the video data itself had no time stamp on it. The frame rate would start at $30 \mathrm{fps}$, then drop through the course of a recording, as low as $18 \mathrm{fps}$. It would take about 10 minutes for the frame rate to drop that low, and it stayed relatively constant for the first few minutes of the video. This problem became progressively worse throughout the 3 hours of recording, but did not affect the first few recordings. This thesis focuses on one CMWIR video that was taken very early in the set, and has been shown not to suffer a significant frame rate degradation. The CMWIR video was shown not to degrade fps by comparing noticeable moving features (for example, a puff of rising smoke, a person walking, a card driving, a bird flying) between the CMWIR video and the visible video, which has a time stamp that proves its constant frame rate.

\subsubsection{Light Bloom}

Light bloom distorts the heat plume. Pixels bleed into their neighbors in proportion to the bleeding pixel's intensity. Since the flame in the scene has a very high intensity, it bleeds into its surroundings and extends well into the heat plume. It has been found that both the flame's intensity and its variation are very high (Section 2.5) compared with the heat plume: flame has a typical intensity of 12,000 and variance that saturates the unsigned 16-bit integer that represents it 
(implying that the variance of flame is greater than $2^{16}=65,536$, which far exceeds anything else in the scene), while heat plume doesn't have a typical intensity (as it brightens the natural background intensity), and has a typical variance of 300 . Hence the light bloom causes the exceedingly high variance of the flame to bleed into the variance measurement of the heat plume. One of the assumptions was that the flame would not be visible. This is a flaw that should be rectified in further studies. 


\section{FEATURES}

A "feature," in this thesis refers to a piece of information local to a pixel coordinate at a given time index. A frame or image of these per-pixel features will also be termed a "feature" or "feature frame," such that context will make it clear. An eclectic combination of features theoretically identifies a unique signature of a heat plume. Since this thesis focuses on temporal signature, the features considered result from operations through time, per pixel. Let a video frame group spanning contiguous frames of dimension R-by-C and length $\mathrm{T}$, starting at time $t_{1}$ and ending at time $t_{f}=t_{1}+T-1$, be represented as a 3-dimensional matrix,

$$
\left.\boldsymbol{G}=\boldsymbol{G}_{t_{f}}=\left[\begin{array}{ccc}
G_{1,1,1} & \cdots & G_{1, C, 1} \\
\vdots & \ddots & \vdots \\
G_{R, 1,1} & \cdots & G_{R, C, 1}
\end{array}\right] \cdots\left[\begin{array}{ccc}
G_{1,1, T} & \cdots & G_{1, C, T} \\
\vdots & \ddots & \vdots \\
G_{R, 1, T} & \cdots & G_{R, C, T}
\end{array}\right]\right] \quad \text { Equation } 1
$$

The cells of a 2-dimensional feature matrix is found with:

$$
f_{r, c}^{\text {feature identifier }}=\text { FeatureFunction }\left\{\left.\left(G_{r, c, t}\right)\right|_{t=[1, T]}\right\} \quad \text { Equation } 2
$$

or, in compact form,

$$
\boldsymbol{F}_{t_{f}}^{\text {feature identifier }}=\mathcal{F}\left(\boldsymbol{G}_{t_{f}}\right)
$$

for general set-input, single output function FeatureFunction. The input to FeatureFunction is the set of all time values for the fixed, generic row $r$ and column $\mathrm{c}$.

Throughout this document, feature frame variables will be expressed as a capital letter with the feature identifier (a short-form string indicating the feature's formal name) in its superscript. If the variable represents the entire frame matrix, it will be in boldface as in Equation 3, and its subscript refers to the absolute time index $t_{f}$ of the last frame within $\boldsymbol{G}_{t_{f}}$. If the variable represents a cell within the matrix, it will be plain-face and its subscripts will identify its position within the matrix, as in Equation 2. Figure 15 illustrates how each pixel of feature frame $\mathbf{F}$ relates to the time-set of the same pixel of the frame group $\mathbf{G}$. 


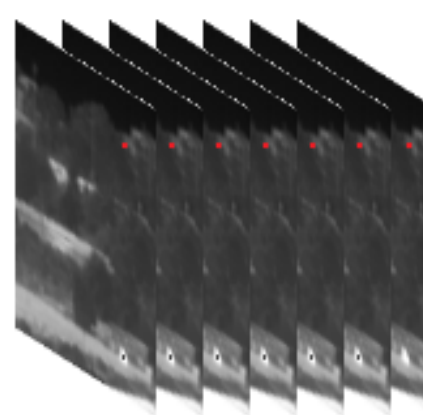

Frame group, $\mathbf{G}$

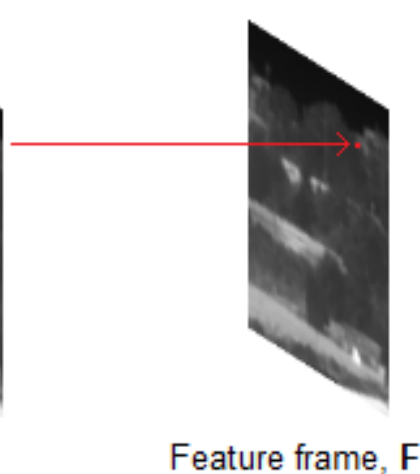

Feature frame, $\mathbf{F}$

Figure 15 - Relationship between Frame Group and Feature Frame

The following sections will present the rationale behind each feature, provide a definition in terms consistent with convention of this thesis and then briefly argue the feature's merit.

\subsection{Statistical Features}

\subsubsection{Variance}

\subsubsection{Rationale}

Variance over time represents information about movement, a defining characteristic of a heat plume. Variance is a commutative figure, so it does not give any information about frequency, only a magnitude of change within the time frame in which it is applied. Many events will register movement in addition to heat plume, such as foliage, cars, people, and birds, but each of these move in different patterns, so time variance carries useful information.

\subsubsection{Definition}

The variance feature frame is the variance over time of a frame group $\mathbf{G}$, per pixel. The FeatureFunction $\mathcal{F}=\operatorname{var}(\{\}$.$) , and the variance frame is described by the following equations.$

$$
F_{r, c}^{v a r}=\operatorname{var}\left(\left.G_{r, c, t}\right|_{t=[1, \mathrm{~T}]}\right)
$$


In detail:

$$
\begin{gathered}
=\frac{1}{t_{l e n}} \sum_{t=1}^{T}\left(G_{r, c, t}-\mu_{r, c}\right)^{2} \\
\mu_{r, c}=\frac{1}{T} \sum_{t=1}^{T} G_{r, c, t}
\end{gathered}
$$

where:

In short:

$$
\begin{gathered}
\boldsymbol{F}^{\text {var }}=\operatorname{var}(\boldsymbol{G}) \\
\boldsymbol{F}_{t_{f}}^{\operatorname{var}}=\operatorname{var}\left(\boldsymbol{G}_{t_{f}}\right)
\end{gathered}
$$

\subsubsection{Analysis}

Figure 16 shows four sub-frames, sampled at a uniform difference of 30 frames apart (approximately 1 second at $30 \mathrm{fps}$ ). Each frame represents the variance of the past 30 frames of original data, so variance frame 3030 is the pixel-wise variance of the range [3001, 3030], represented as $\boldsymbol{G}_{3030}$. A variance image shows the presence of the heat plume, as well as other areas, like foliage, and light-dark interfaces due to motion jitter that video stabilization (Section 2.4) could not completely correct. But the heat plume signal is strong in the variance image, and varies more with time than the other background noise sources, making variance a useful feature.

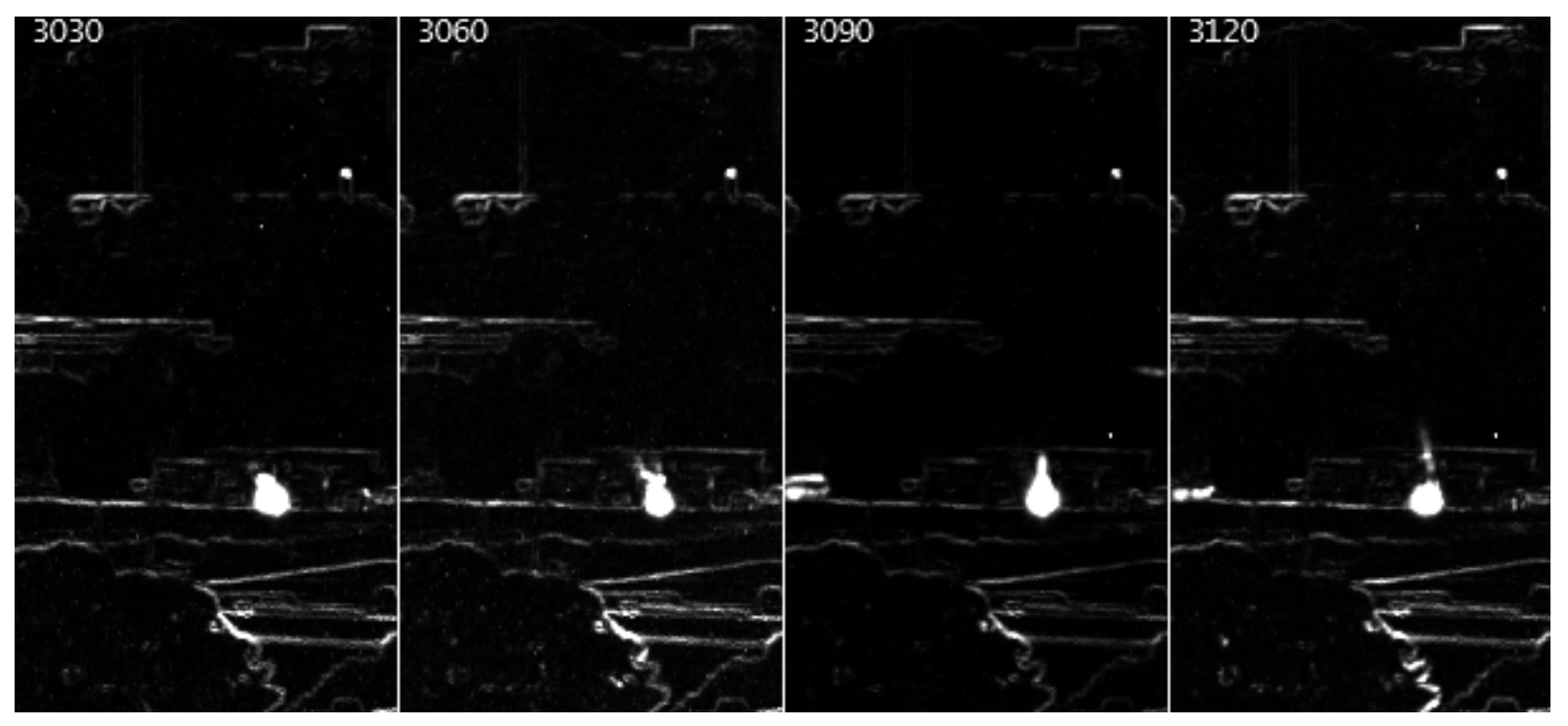

Figure 16 - Variance Feature Frames Sample

(Stabilized oats1 channel, frame indices shown) 
Section 5.2.2 digs deeper into the variance feature with an experiment.

Table 7 - Variance Feature Function Descriptions

\begin{tabular}{|c|c|c|}
\hline \multirow[t]{2}{*}{ Function / Script Name } & \multicolumn{2}{|c|}{ Description Overview } \\
\hline & Function Input & Function Output \\
\hline FeatureExtraction_Var & $\begin{array}{l}\text { Script that extracts vari } \\
\text { channel, given time ran }\end{array}$ & $\begin{array}{l}\text { from a given video } \\
\text { n region of interest. }\end{array}$ \\
\hline
\end{tabular}

\subsubsection{Variance Set Statistics (VSS)}

\subsubsection{Rationale}

The variance frames, when seen side-by-side (Figure 16), show the progression of a heat plume. Out of the whole scene, the heat plume changes shape the most drastically. Where the heat plume passes, the maximum of a variance will be highly affected, where the mean will be less affected. Contrast the heat plume with stationary noise in the foreground, where the mean and the max will not differ as much. Therefore, a combination of the mean and maximum variance data should give information that can discriminate between heat plume and other background elements.

\subsubsection{Definition}

Variance set statistics refers to statistical operations on a set of uniformly time-separated variance frames. For a set of size $\mathrm{SN}$ and a constant time step $\Delta \mathrm{t}$ :

$$
\begin{aligned}
& \left.\boldsymbol{S}_{t_{f}}=\left[\begin{array}{ccc}
S_{1,1,1} & \cdots & S_{1, C, 1} \\
\vdots & \ddots & \vdots \\
S_{R, 1,1} & \cdots & S_{R, C, 1}
\end{array}\right] \cdots\left[\begin{array}{ccc}
S_{1,1, T} & \cdots & S_{1, C, T} \\
\vdots & \ddots & \vdots \\
S_{R, 1, T} & \cdots & S_{R, C, T}
\end{array}\right]\right] \\
& =\left[\begin{array}{llll}
F_{1}^{v a r} & F_{2}^{v a r} & \cdots & F_{S N}^{v a r}
\end{array}\right] \\
& \left.=\left[\begin{array}{ccc}
\left(F_{t_{f}-S N+1}^{v a r}\right)_{1,1} & \cdots & \left(F_{t_{f}-S N+1}^{v a r}\right)_{1, C} \\
\vdots & \ddots & \vdots \\
\left(F_{t_{f}-S N+1}^{v a r}\right)_{R, 1} & \cdots & \left(F_{t_{f}-S N+1}^{v a r}\right)_{R, C}
\end{array}\right] \cdots\left[\begin{array}{ccc}
\left(F_{t_{f}}^{v a r}\right)_{1,1} & \cdots & \left(F_{t_{f}}^{\text {var }}\right)_{1, C} \\
\vdots & \ddots & \vdots \\
\left(F_{t_{f}}^{\text {var }}\right)_{R, 1} & \cdots & \left(F_{t_{f}}^{\text {var }}\right)_{R, C}
\end{array}\right]\right]
\end{aligned}
$$




$$
\boldsymbol{F}_{t+1}^{v a r}=\operatorname{var}\left(\boldsymbol{G}_{t+\Delta t}\right)
$$

The set $\mathrm{S}$ is represented as a matrix for simplicity, though order does not matter, as of a set. The time index of $\boldsymbol{F}_{S N}^{v a r}$ corresponds to $t_{f}$ for time identification purposes. That is, the variance set and the derived variance set statistic frames will be indexed at the time corresponding to the last frame in the set at index SN. Variance set statistics used in this thesis are mean and max, which are computed per-pixel over the entire frame set:

$$
\begin{array}{rlr}
\operatorname{varMean}_{r, c}\left(\boldsymbol{S}_{t_{f}}\right) & =\operatorname{mean}\left\{S_{r, c, 1}, S_{r, c, 2,}, \ldots, S_{r, c, T}\right\} & \\
& \rightarrow \operatorname{varMean}_{t_{f}} & \\
\operatorname{varMax}_{r, c}\left(\boldsymbol{S}_{t_{f}}\right) & =\max \left\{S_{r, c, 1}, S_{r, c, 2,}, \ldots, S_{r, c, T}\right\} & \\
& \rightarrow \operatorname{varMax}_{t_{f}} & \text { Equation 6 }
\end{array}
$$

The feature frames $\boldsymbol{v a r M e a n}_{t_{f}}$ and $\boldsymbol{v a r M a x}_{t_{f}}$ are matrices whose elements are defined by Equation 5 and Equation 6, respectively. Recall that the subscripts on varMean, varMax, S, and $\mathbf{G}$ refer to the final time index represented by the frame group $\boldsymbol{G}_{\boldsymbol{t}_{\boldsymbol{f}}}$. Figure 17 illustrates how $\operatorname{varMean}_{t_{f}}$ and $\operatorname{varMax}_{t_{f}}$ are calculated. 


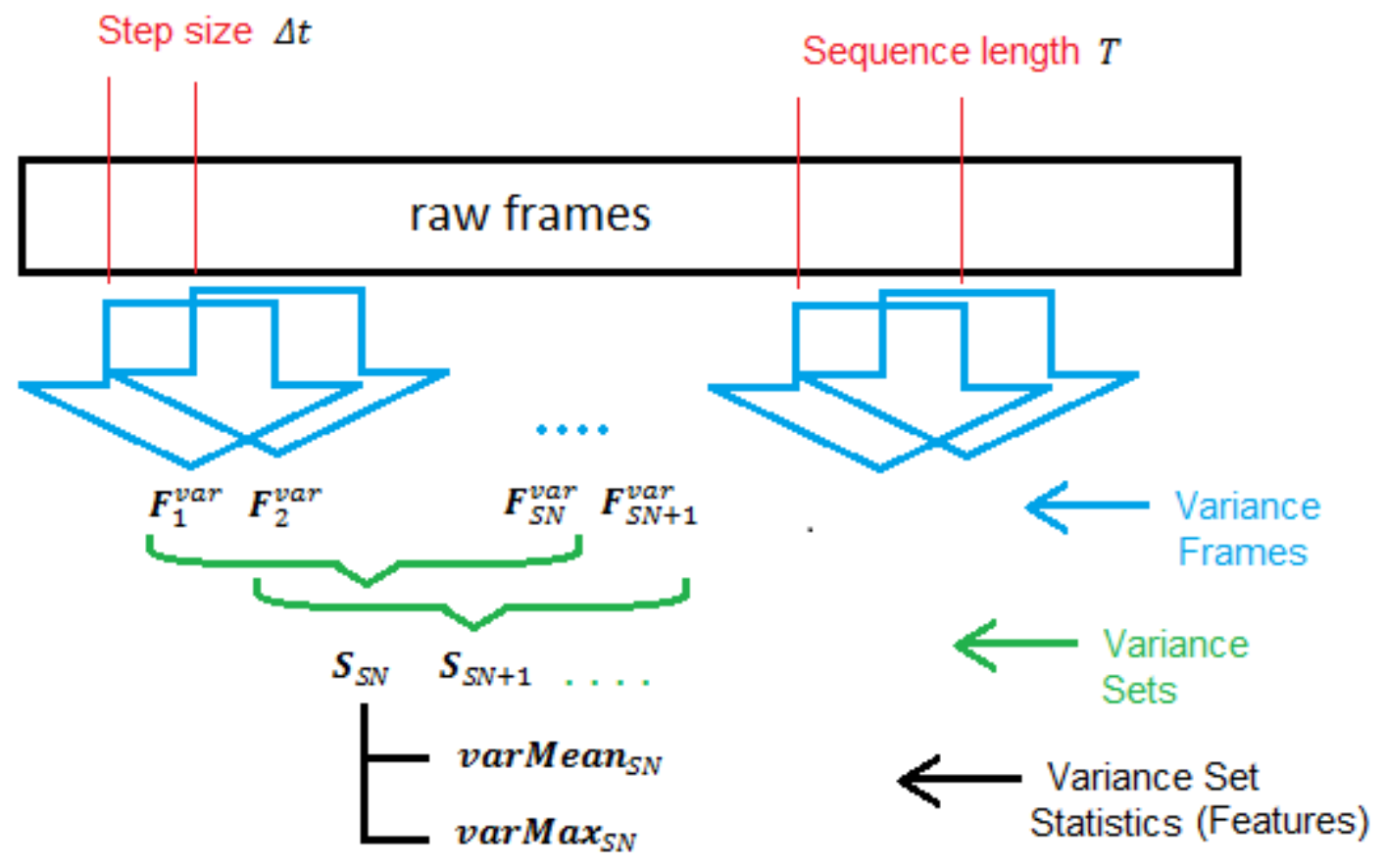

Figure 17 - Variance Set Statistics Visual Aid

\subsubsection{Analysis}

To test these features, variance samples were taken from the pine_cones video (pine_cones_PFstab), from pixels near the heat plume and other high- and low-movement areas for comparison, and various statistics were calculated. Figure 18 shows the locations of the masks used to select these regions, and Table 8 shows the statistics and names of the regions, with data from oats 1 video. Table 9 shows more statistics from the pre-stabilized pine_cones video. The masks were created by thresholding a variance frame over 200 frames, to identify regions of greatest movement posing the greatest challenge to heat plume detection. The data are from sequences of length 30, spanning a range of 220 frames. The number of samples from each mask is directly proportional to the mask's area, hence the heat region has the fewest samples. 


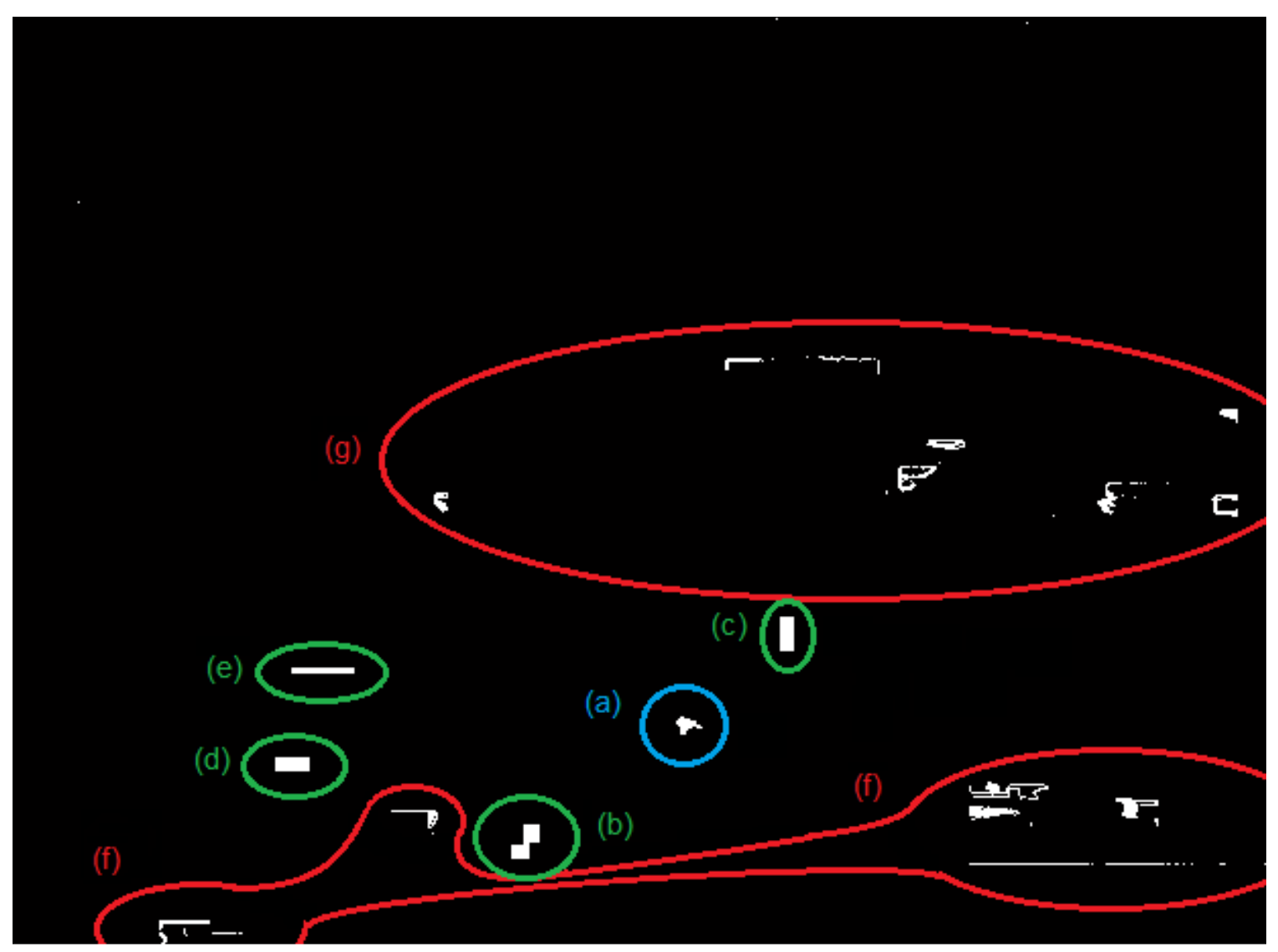

Figure 18 - Mask Locations for Data Sampling
(a) Heat plume
(b) Near foliage
(c) Mid-ground foliage 1
(d) Mid-ground foliage 2
(e) Grassy field
(f) Near noise
(g) Distant noise

Table 8 - Variance Set Statistics Defined for Figure 18 Masks (Statistics from oats1 video, frames . Standard deviation shown for convenience)

$\begin{array}{llllll}\begin{array}{l}\text { STANDARD DEVIATIONS OF } \\ \text { Min }\end{array} & \text { Mean } & \text { Median } & \text { Max } & \text { Samples } & \\ 1.89 & 4.8 & 4.5 & 10.2 & 348 & \text { (a) Heat Plume } \\ 1.87 & 3.5 & 3.3 & 12.2 & 828 & \text { (b) Near Foliage } \\ 1.57 & 3.9 & 3.0 & 46.4 & 714 & \text { (c) Mid-Ground Foliage } \\ 1.61 & 3.0 & 2.9 & 5.4 & 582 & \text { (d) Mid-Ground Foliage2 } \\ 1.92 & 3.8 & 3.4 & 12.6 & 714 & \text { (e) Grassy Field } \\ 1.61 & 7.2 & 4.2 & 98.3 & 3438 & \text { (f) Near Noise } \\ 1.03 & 3.9 & 3.1 & 19.8 & 2802 & \text { (g)Distant Noise }\end{array}$


Table 9 - More Variance Set Statistics (From pre-stabilized pine_cones video, frames 20002200.)

$\begin{array}{llllll}\text { STANDARD } & \text { DEVIATIONS OF } & \text { SEQUENCES } & & \\ \text { Min } & \text { Mean } & \text { Max } & \text { GeoMean } & \text { Samples } & \\ 2.51 & 11.06 & 47.95 & 9.74 & 642 & \text { Heat Plume } \\ 2.55 & 14.04 & 77.95 & 10.12 & 840 & \text { Near Foliage } \\ 2.02 & 13.51 & 119.29 & 6.68 & 666 & \text { Mid-Ground Foliage } \\ 1.43 & 4.42 & 17.08 & 4.25 & 864 & \text { Grassy Field }\end{array}$

From the numbers, the samples appear different, especially in the pre-stabilized pine_cones video. When varMean and varMax frames are opened and their histograms examined, thresholds can be found that bound the heat plume area, with different content. Table 10 shows a few snapshots of the mean and max features at different thresholds around the barbecue pit.

\section{Table 10 - Variance Statistics Threshold Snapshots}

Feature Name

varMax_seq30_set20:

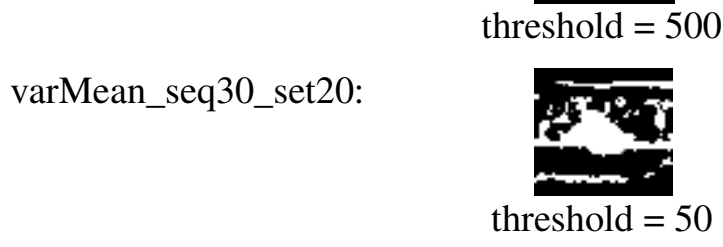

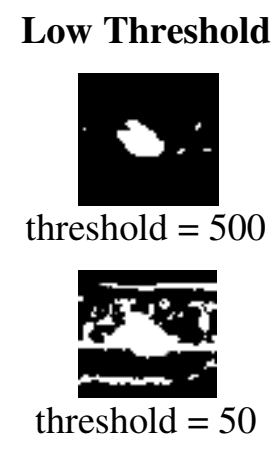

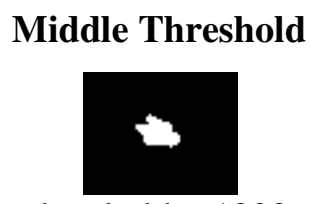

threshold $=1200$

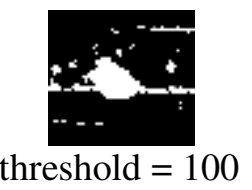

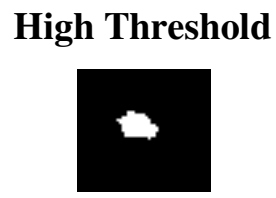

threshold $=2500$

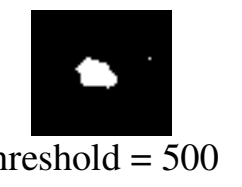

The varMean feature contains much movement from heat and other background areas, where the varMax feature is more focused around the fire, because the fire varies wildly. Section 5.3 will develop these features into a classifier.

Figure 19 shows time-sequences of pixels from three regions shown in Figure 18, namely the grassy field (e), foreground foliage (b), and the heat plume (a). Grass has little time variation and little ensemble variation among pixel means; foliage has much greater variation in both time and ensemble; smoke has a high ensemble variation, as its background covers a variety of shades, and a medium time variation, as the smoke only gently brightens the natural background pixel shade as the heat waves travel in the breeze. The high ensemble variation in the heat comes from 
the background behind the heat, and not the heat itself. The best marker for a variance-only classifier would be a window of standard deviation that matches this smoke.
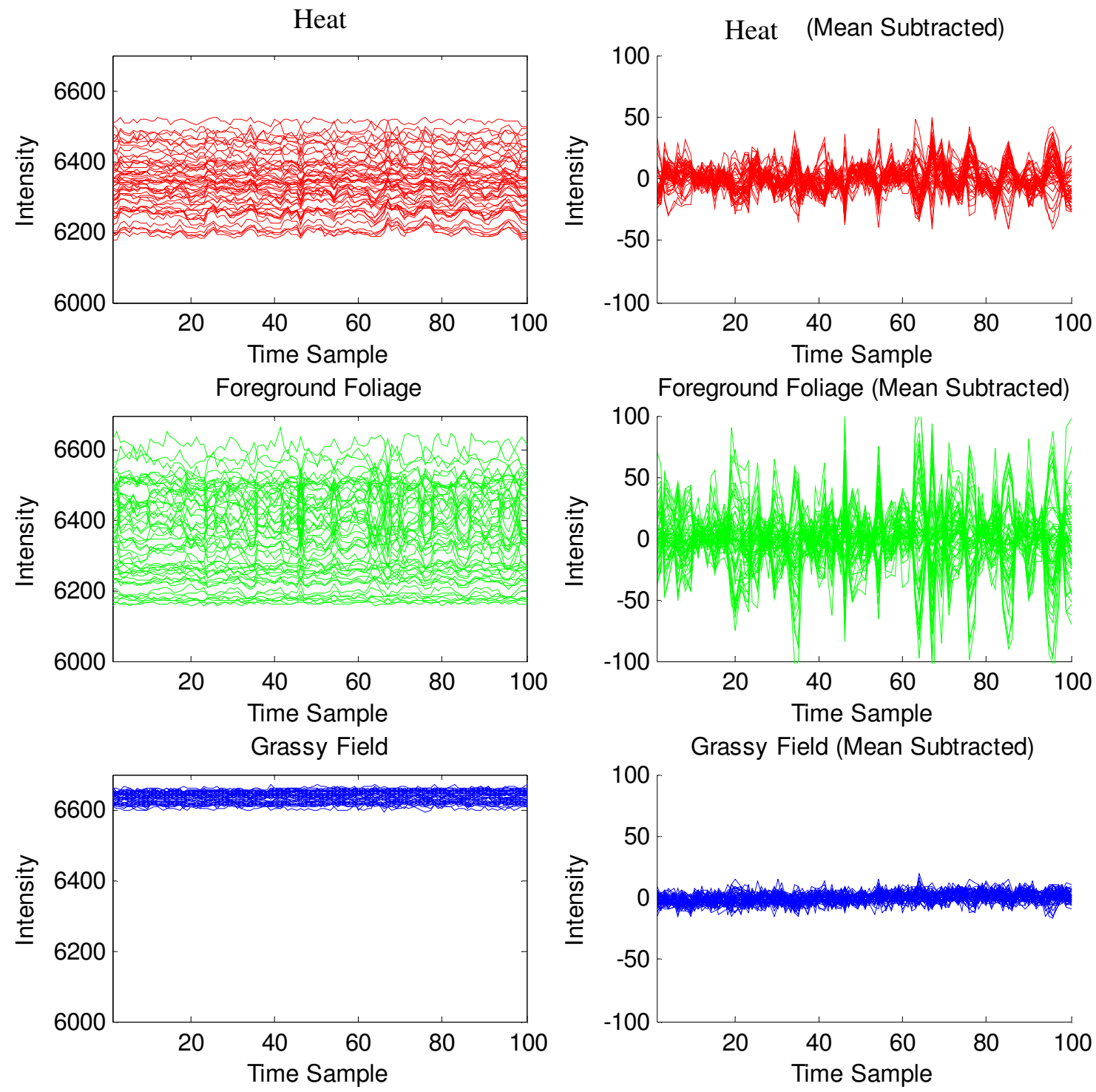

Figure 19 - Time Sequences of Pixels by Region. Top/Red: Figure 18(c). Middle/Green: Figure 18(b). Bottom/Blue: Figure 18(e) 


\subsubsection{Simple Moving Average (SMA)}

\subsubsection{Rationale}

A moving average represents behavior on a longer time-scale. Every frame of a moving average represents information from the present and the past. Representing past information in the present necessarily contributes to identifying a temporal signature.

\subsubsection{Definition}

The simple moving average was already partially seen in Equation 5 from the variance feature frame derivation.

$$
\begin{aligned}
& F_{r, c}^{S M A}= \operatorname{mean}\left(\left.G_{r, c, t}\right|_{t=[1, \mathrm{~T}]}\right) \\
& \rightarrow \boldsymbol{F}_{t_{f}}^{S M A}
\end{aligned}
$$

Consistent with the convention so-far developed, the subscript $t_{f}$ of the SMA-feature frame matrix $\boldsymbol{F}_{t_{f}}^{S M A}$ refers to the time of the last frame of $\mathbf{G}$.

\subsubsection{Merit}

SMA shows a general trend over its time range $\mathrm{T}$, mitigates fast changes as low-pass filter. Reduces background noise due to camera intensity resolution.

\subsubsection{Exponentially Weighted Moving Average (EWMA or EMA)}

\subsubsection{Rationale}

Like SMA, EWMA represents information over past time in the current frame, but instead of representing a range of time uniformly, it does so exponentially, weighting recent information more heavily than older information. It will reduce noise while responding more quickly to changes in the signal. Its frequency response is smooth, where the SMA has prominent side lobes (Figure 20). Furthermore, the EWMA can be expressed recursively, an infinite impulse 
response (IIR) filter, hence it can be computed in a progressive manner far more efficiently. Figure 21 shows the similarity of the time domain response of the SMA and EWMA filters.

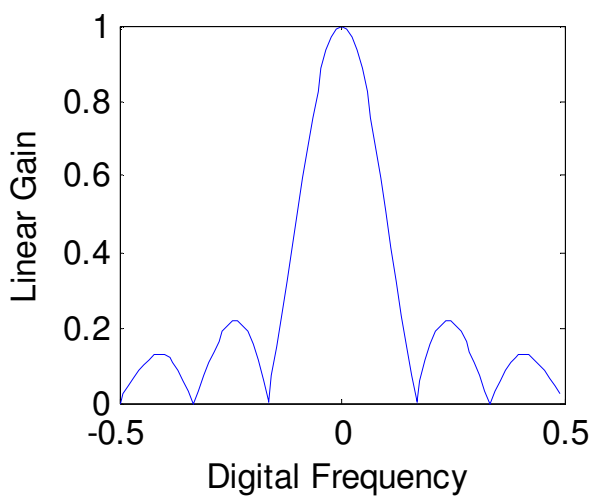

(a) SMA

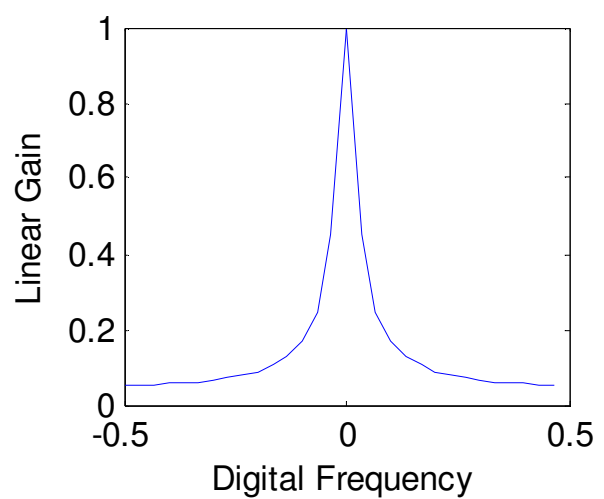

(b) EWMA

Figure 20 - Comparison of Frequency Response Magnitude of SMA and EWMA $($ SMA length $=20$, EWMA p-value $=0.1)$

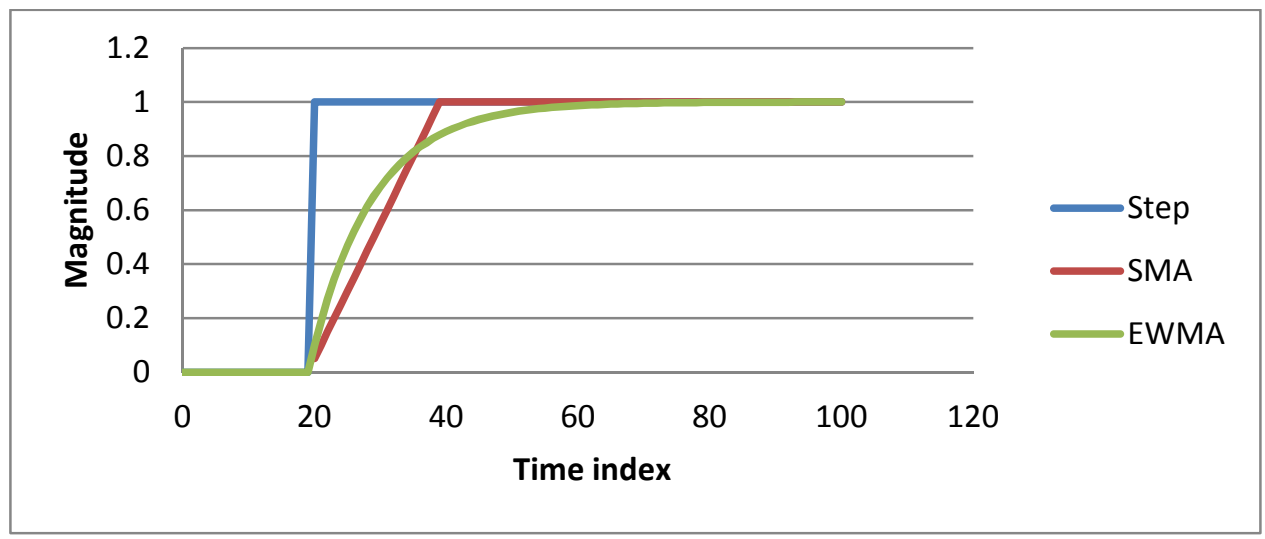

Figure 21 - Comparison of SMA and EWMA on Step Function $($ SMA length $=20$, EWMA p-value $=0.1)$

The SMA filter had length 20, and the EWMA filter's p-value was 0.1.

It can be shown that the EWMA-subtracted signal is proportional to the moving difference (discrete derivative). 


\subsubsection{Definition}

Unlike other features, the EWMA is not defined for a range of time frames, G. Instead it is defined recursively as a linear combination of its previous value, and the current observation.

Per pixel: $\quad\left(F_{r, c}^{E W M A}\right)_{t}=p \cdot X_{r, c}+(1-p) \cdot\left(F_{r, c}^{E W M A}\right)_{t-1}$

Per frame: $\quad \boldsymbol{F}_{t}^{E W M A}=p \cdot \boldsymbol{X}+(1-p) \cdot \boldsymbol{F}_{t-1}^{E W M A}$

The parameter $\mathrm{p}$ determines its sharpness or range, analogously to how $\mathrm{T}$ determined the sharpness or range of the SMA. A larger $\mathrm{p}$ means the current observation weights more than the past observation, so the filter allows higher-frequency content.

\subsubsection{Dual-Range Moving Average Difference (DRMAD)}

\subsubsection{Rationale}

The scene contains much noise, and most of the noise comes from predictable sources, such as foliage and contrasting interfaces in the presence of jitter. These sources have high variance, but they do not migrate from their locality. A pixel measurement can be seen as a sum of signal and predictable noise [14]. Or, expressed in terms of frames:

$\begin{array}{rlr} & \boldsymbol{f}=\boldsymbol{s}+\boldsymbol{n} & \\ \text { Then: } & \boldsymbol{s}=\boldsymbol{f}-\boldsymbol{n} & \text { Equation 7 }\end{array}$

where $\boldsymbol{f}$ is the measurement, $\boldsymbol{s}$ is the ideally detected heat plume signal, and $\boldsymbol{n}$ is a noise prediction.

A moving average shows a general trend, which would include intensity trends. Treating a long-range moving average as a noise predictor, and subtracting it from the moving signal will cancel out constant elements of the scene and show only the moving elements. A short-term moving average will reduce random measurement noise, creating a smoother image, more comparable to the long-range moving average, clarifying the output. 


\subsubsection{Definition}

The DRMAD will be defined in terms of the EWMA, because of its smooth frequency response and quick computation time.

Per pixel:

$$
\left(F_{r, c}^{D R M A D}\right)_{t}=\left(F_{r, c}^{E W M A_{\text {short }}}\right)_{t}-\left(F_{r, c}^{E W M A_{\text {long }}}\right)_{t}
$$

Per frame:

$$
\boldsymbol{F}_{t}^{D R M A D}=\boldsymbol{F}_{t}^{E W M A_{\text {short }}}-\boldsymbol{F}_{t}^{E W M A_{\text {long }}}
$$

The "short" and "long" subscripts indicate a larger $p$-value and smaller $p$-value, respectively, where $p \leq 0.1$. For simplicity, a DRMAD will be referred to in terms of its $p$-values in short form:

$$
\operatorname{EWMA}\left(p_{\text {short }}\right)-\left(p_{\text {long }}\right)
$$

For example:

EWMA0.07-0.02 refers to EMWA of $p=0.07$ less EWMA of $p=0.02$, of a given input channel.

\subsubsection{Merit}

Figure 22 compares multiple DRMADs based on EWMA. The heat plume has relatively high strength compared with the background. There are still weaker signals in the background, especially in the high-contrast interfaces, but not as obvious as the heat plume. Since DRMAD is a difference, it will have positive and negative numbers. For display purposes, a linear mapping is applied such that zero maps to mid-grey, and the absolute largest magnitude maps to 1 . 


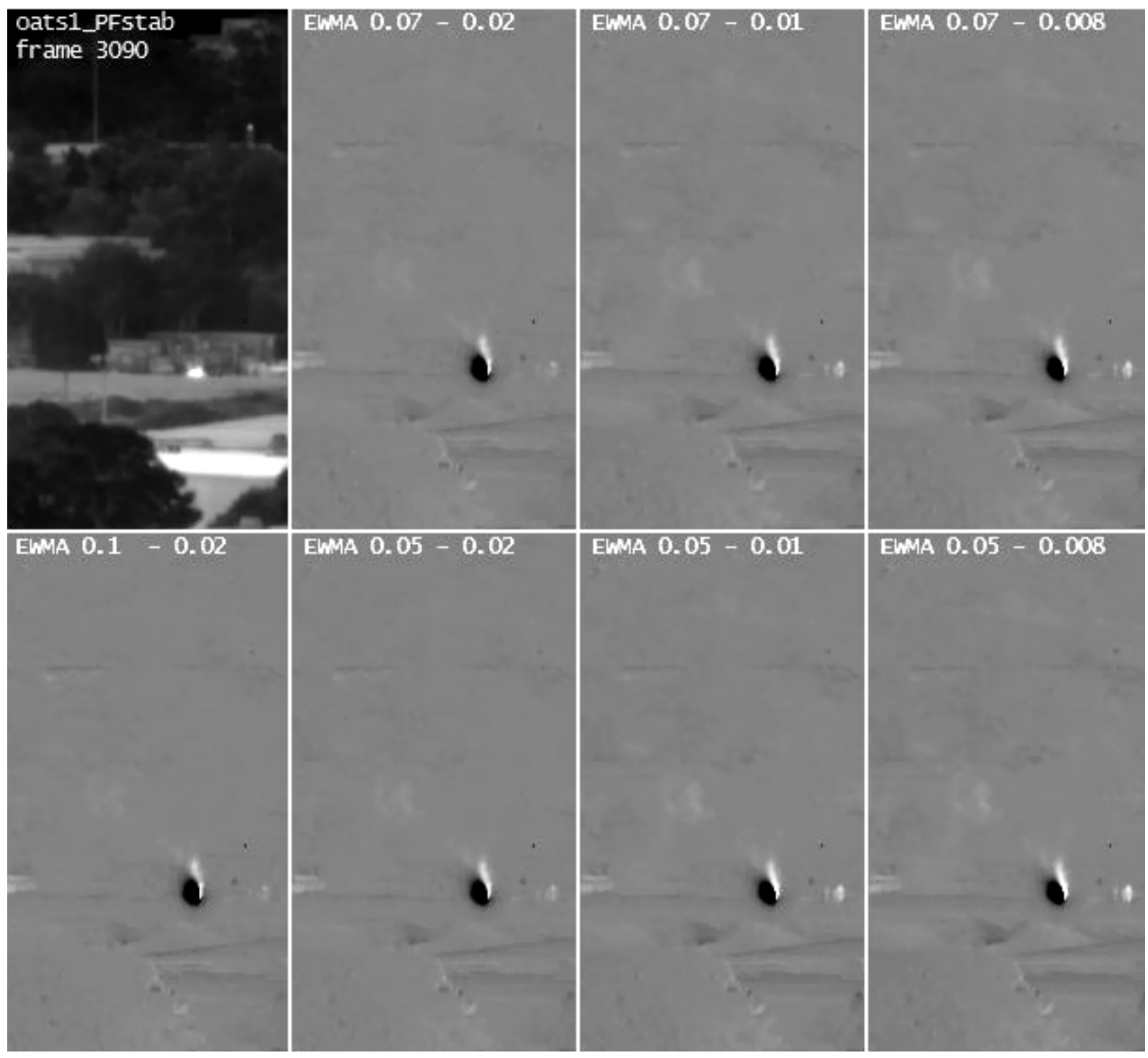

Figure 22 - Comparison of Dual Range Exponentially Weighted Moving Average Difference (DRMAD) pairs (oats1 stabilized channel, e.g. lower left pane is EWMA of pvalue $=0.1$ less EWMA of p-value $=0.02$ )

\subsection{Principal Component Analysis (PCA)}

\subsubsection{Rationale}

Principal Component Analysis (also known as the Karhunen-Loeve transform, or KLT) transforms the dimensions of input samples into new dimensions such that the variance is maximized along the first basis vector in PC-space, and the subsequent orthogonal basis vectors 
point in the direction of the next greatest variance [17]. In this way, most of the variance lies within the first few dimensions. PCA has the notable property of de-correlation. To wit, every dimension of a given data in PC-space has no correlation with any other PC-space dimension. Equivalently, the covariance matrix becomes non-zero only in the main diagonal. PCA can then be used to express data in a unique way that reveals movement information. For the purpose of uncovering movement over time, PCA will be performed over the time dimension, or, as it is known in literature, "multi-temporal PCA." The first-order principle component is a measure of persistence, and the deeper orders accompany growth.

\subsubsection{Derivation}

In general, PCA generates a Principal Component Transform (PCT) W, which maps data in input space to data in PC-space:

$$
\boldsymbol{Y}=\boldsymbol{X} \cdot \boldsymbol{W}
$$

Equation 9

Where $\mathbf{X}$ and $\mathbf{Y}$ are $(\mathrm{m}, \mathrm{n})$ matrices, and $\mathbf{W}$ is a $(\mathrm{n}, \mathrm{n})$ matrix. (Note: In popular literature, $\mathrm{X}$ and $Y$ and $W$ are represented as the transpose of what they are represented as here, which only complicates the derivation and application, and increases computation time, solely for the sake of representing the transform as a traditionally pre-multiplied matrix.)

PCA applies to any one dimension of an initial given data set, and does not discriminate between the other dimensions (this will be explained shortly). For the purpose of extracting temporal information, PCA will be performed along the time dimension, and the pixel locations will have no bearing on the Principal Component Transform (PCT); row and column will be temporarily grouped together, preserving the order. Let the columns of $\mathbf{X}$ be the rows and columns of $\mathbf{G}$, reshaped into $(\mathrm{R} \cdot \mathrm{C}, 1)$ column vectors, for each time $t \in[1, T]$. Then $\mathbf{X}$ will be dimension $(\mathrm{M}=\mathrm{R} \cdot \mathrm{C}, \mathrm{N}=\mathrm{T})$.

$$
\boldsymbol{G}_{R, C, T} \leftrightarrow \boldsymbol{X}_{R \cdot C, T}
$$

Equation 10

Similarly: $\quad \boldsymbol{P}_{R, C, T} \leftrightarrow \boldsymbol{Y}_{R \cdot C, T}$ 
This re-dimensioning is exactly as illustrated in Figure 7 (d). $\mathbf{X}$ and $\mathbf{Y}$ are both simply frame groups where each frame is represented as a column vector with order preserved.

The steps to derive the Principal Component Transform $\mathbf{W}$ are as follows:

1. Compute the covariance matrix, $\boldsymbol{C}_{\boldsymbol{X}}$, of the input data set $\mathbf{X}$.

$$
C_{X}=\frac{1}{R \cdot C} \boldsymbol{X} \cdot \boldsymbol{X}^{T}
$$

2. Compute the $\mathbf{N}$ Eigenvectors $\mathbf{V}_{\mathbf{i}}$ of the covariance matrix $\boldsymbol{C}_{\boldsymbol{X}}$, arranged in decreasing order of associated Eigenvalue, $\lambda_{\mathrm{i}}$.

3. Let the columns of $\boldsymbol{W}$ be the Eigenvectors $\mathbf{V}_{\mathbf{i}}$.

$$
\boldsymbol{W}=\left[\begin{array}{llll}
\boldsymbol{V}_{1} & \boldsymbol{V}_{2} & \ldots & \boldsymbol{V}_{N}
\end{array}\right]
$$

The PCT transforms the observations $\mathbf{X}$ from input space to observations $\mathbf{Y}$ in PC-space, such that the covariance matrix in $\mathrm{PC}$-space $\boldsymbol{C}_{\boldsymbol{Y}}$ is a diagonal matrix, where the diagonals are the Eigenvalues $\lambda_{\mathrm{i}}$ of $\boldsymbol{C}_{\boldsymbol{X}}$.

$$
\boldsymbol{C}_{\boldsymbol{Y}}=\operatorname{diag}(\boldsymbol{\lambda})=\left[\begin{array}{ccc}
\lambda_{1} & \cdots & 0 \\
\vdots & \ddots & \vdots \\
0 & \cdots & \lambda_{N}
\end{array}\right]
$$

The zeros on the non-diagonals of $\boldsymbol{C}_{\boldsymbol{X}}$ agrees with the earlier statement that the dimensions in PCspace have no correlation with each other.

To view the output $\mathbf{Y}$, it must be re-ordered to recover the spatial relationship between pixels. Again, refer to Figure $7(\mathrm{~d})$ to see the relationship between $\mathbf{Y}(\mathrm{R} \cdot \mathrm{C}, \mathrm{T})$ and $\mathbf{P}(\mathrm{R}, \mathrm{C}, \mathrm{T})$ (Equation 10). The frames within $\mathbf{P}$ are referred to as Principle Component Images (PCI).

\subsubsection{Method}

For a frame group $\mathbf{G}$ that windows through a video sequence, its frames are selected at a constant frame interval $\Delta t$. The process is as follows:

1. Assign the initial frame group $\boldsymbol{G}$ 
2. Compute the PCT, $\boldsymbol{W}$ (Equation 11) on initial $\boldsymbol{G}$

3. Loop through the video, progressively windowing $\boldsymbol{G}_{t}$. For each $\boldsymbol{G}_{t}$,

a. Map $\boldsymbol{G}_{t}$, to $\boldsymbol{X}_{t}$ (Equation 10)

b. Transform $\boldsymbol{X}_{t}$ to $\boldsymbol{Y}_{t}$ using the same $\boldsymbol{W}$ from step 2 (Equation 11)

c. Map $\boldsymbol{Y}_{t}$ to $\boldsymbol{P}_{t}$

This procedure assumes a stationary field of view, so that the principal component vectors (columns of $\mathbf{W}$ ) map sets of input pixels to principal component space for any frame group. Furthermore, if thresholds can be found that enclose heat plume motion, those thresholds can apply to the entire video.

Figure 23 shows an example of PCA on 6 frames from a DRMAD channel (EWMA0.07 - 0.02). Each frame is independently auto-linear intensity mapped to show content.

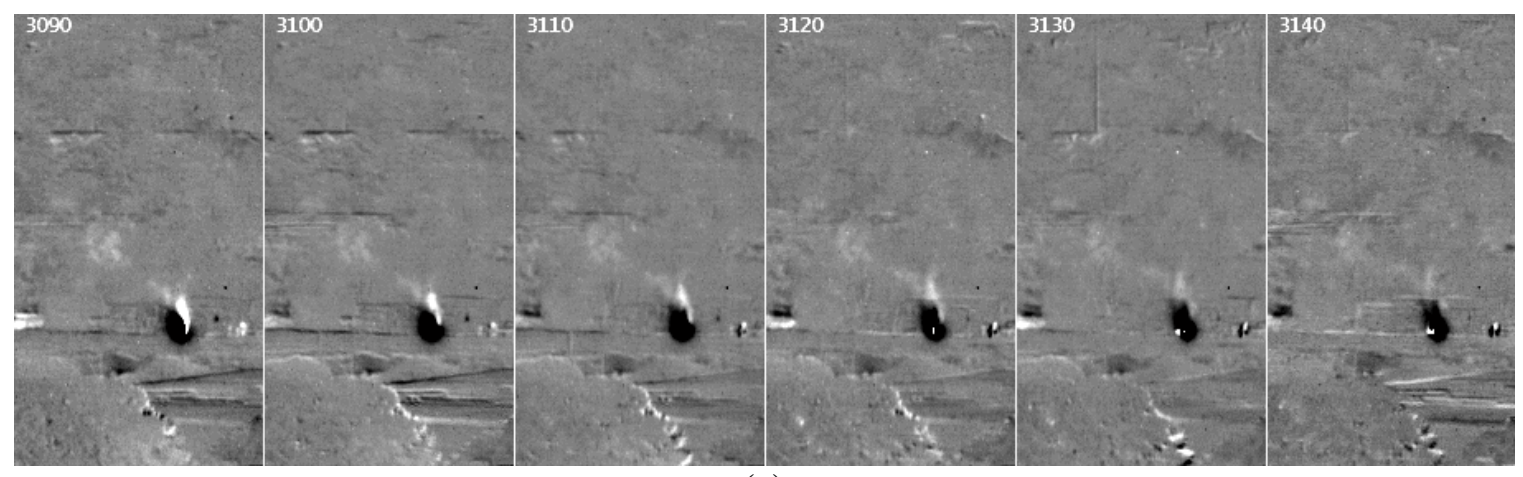

(a)

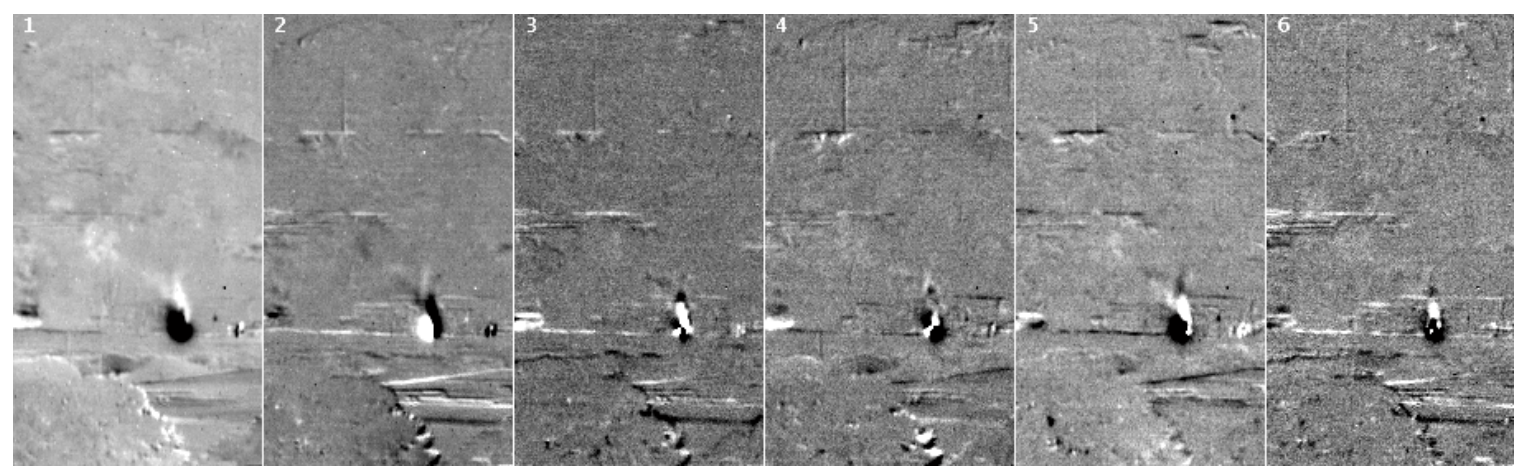

(b)

Figure 23 - Example of PCA (b) on Input Frames (a) from oats1 DRMAD (EWMA0.07-0.02_PCA6_autolinear, non-uniform linear map per PC) 


\subsection{Feature Function Summary}

\subsubsection{Feature Extraction}

Table 11 includes all functions referenced in Section 2.6, to summarize in one place for the reader's convenience, as well as other functions used directly or indirectly.

Table 11 - Feature Extraction Function Descriptions

\begin{tabular}{|c|c|c|}
\hline \multirow[t]{2}{*}{ Function / Script Name } & \multicolumn{2}{|r|}{ Description Overview } \\
\hline & Function Input & Function Output \\
\hline $\begin{array}{l}\text { FeatureExtraction } \\
\text { _EWMA }\end{array}$ & $\begin{array}{l}\text { Source name, } \mathrm{p}- \\
\text { parameter of EWMA } \\
\text { filter. }\end{array}$ & $\begin{array}{l}\text { Creates a new folder in the associated } \\
\text { Features folder containing the EWMA } \\
\text { of the input data. }\end{array}$ \\
\hline $\begin{array}{l}\text { FeatureExtraction } \\
\text { _SMA }\end{array}$ & $\begin{array}{l}\text { Source name, SMA } \\
\text { length. }\end{array}$ & $\begin{array}{l}\text { Creates a new folder in the associated } \\
\text { Features folder containing the EWMA } \\
\text { of the input data. }\end{array}$ \\
\hline $\begin{array}{l}\text { FeatureExtraction } \\
\text { _VarStats }\end{array}$ & $\begin{array}{l}\text { Source name, sequence } \\
\text { length, set size. }\end{array}$ & $\begin{array}{l}\text { Creates new folders in the associated } \\
\text { Features folder containing mean, } \\
\text { median, and maximum of the moving } \\
\text { variance set. }\end{array}$ \\
\hline $\begin{array}{l}\text { FeatureExtraction } \\
\text { _Var }\end{array}$ & \multicolumn{2}{|c|}{$\begin{array}{l}\text { Script that extracts variance frames from a given video channel, } \\
\text { given time range, and given region of interest. }\end{array}$} \\
\hline extractTrainingData & $\begin{array}{l}\text { Video channel path, } \\
\text { save path, mask path, } \\
\text { frame range and step, } \\
\text { sequence length. }\end{array}$ & $\begin{array}{l}\text { Collects time-sequences from specified } \\
\text { video channel over specified frame } \\
\text { range, of specified sequence length, } \\
\text { beneath specified mask, and writes } \\
\text { them to specified path. }\end{array}$ \\
\hline $\begin{array}{l}\text { extractTrainingData } \\
\text { Batch }\end{array}$ & \multicolumn{2}{|c|}{$\begin{array}{l}\text { Script that calls extractTrainingData in a configurable batch. One } \\
\text { convenient launching point for data extraction. Prints report on } \\
\text { how many sequences were collected from each source. }\end{array}$} \\
\hline TimeDomainAnalysis & \multicolumn{2}{|c|}{$\begin{array}{l}\text { Configurable script that reads the sequence data files that } \\
\text { extractTrainingData saves, and calculates the statistics on them. } \\
\text { Has the optional functionality to plot histograms, create } \\
\text { exponential histogram bins, and save them (not used in this } \\
\text { section). }\end{array}$} \\
\hline computePCT & Input frames $(\mathrm{R} \cdot \mathrm{C}-\mathrm{by}-\mathrm{T})$ & $\begin{array}{l}\text { Returns the Principal Component } \\
\text { Transform, W. }\end{array}$ \\
\hline
\end{tabular}




\subsubsection{Feature Processing}

The Feature Processing family of functions converts features into other features by applying per-frame operations, such as median filtering. Each one creates a new folder in the Features folder of a given video channel, and populates it with frames resulting from the specific process applied to a given source folder.

Table 12 - Feature Processing Function Descriptions

\begin{tabular}{|c|c|c|}
\hline \multirow{2}{*}{$\begin{array}{c}\text { Function / Script } \\
\text { Name }\end{array}$} & \multicolumn{2}{|c|}{ Description Overview } \\
\hline & Function Input & Function Output \\
\hline $\begin{array}{l}\text { FeatureProcessing } \\
\text { _MeanSub }\end{array}$ & $\begin{array}{l}\text { Path to two raw data channels, } \\
\text { output path, frame range, } \\
\text { negMethod (what to do when } \\
\text { difference }<0 \text {. 'retain', 'reflect', } \\
\text { 'saturate' }\end{array}$ & $\begin{array}{l}\text { No output. Performs } \\
\text { synchronous frame-wise } \\
\text { subtraction and writes the } \\
\text { difference to specified } \\
\text { output channel folder }\end{array}$ \\
\hline $\begin{array}{l}\text { FeatureProcessing } \\
\text { _PCA }\end{array}$ & $\begin{array}{l}\text { Path to input and output feature } \\
\text { folders, PCA parameters, intensity } \\
\text { pre-mapping specification. }\end{array}$ & $\begin{array}{l}\text { No output. Performs PCA on } \\
\text { the input and saves in output } \\
\text { feature folder with a nested } \\
\text { folder for each PC image. }\end{array}$ \\
\hline $\begin{array}{l}\text { FeatureProcessing } \\
\text { _Simple }\end{array}$ & $\begin{array}{l}\text { Path to input and output feature } \\
\text { folders and a function handle (may } \\
\text { be a Matlab inline function) }\end{array}$ & $\begin{array}{l}\text { Applies the handled function } \\
\text { to each frame, and saves it in } \\
\text { a new feature folder. }\end{array}$ \\
\hline
\end{tabular}




\section{INTENSITY MAPPING}

Some features benefit from intensity mapping either because the range of interest lies within a small range of the data type, or because the data spans both positive and negative values, which can't be plotted. A nonlinear mapping can increase contrast in an image, making the information content more observable to the human observer [15].

The mapping equations presented here may be used by algorithms in Chapter 5, especially in PCA (Section 5.5). This chapter presents the mappings as a reference, so that increased brevity may follow.

\subsection{Linear}

A linear map may be completely described by a mapping of two points $x_{1}$ and $x_{2}$ to another two points $y_{1}$ and $y_{2}$, respectively. Or, equivalently, of one specific range to another specific range, such that $y=m \cdot x+b$. Reference any elementary algebra text.

$$
\begin{gathered}
m=\frac{y_{2}-y_{1}}{x_{2}-x_{1}} \\
b=y_{1}-m \cdot x_{1}=y_{2}-m \cdot x_{2}
\end{gathered}
$$

All that remains is to select the values of $x_{1}, x_{2}, y_{1}, y_{2}$, to derive the mapping parameters, $\mathrm{m}$ and $\mathrm{b}$, to perform the mapping that will be denoted as $\left[x_{1}, x_{2}\right] \rightarrow\left[y_{1}, y_{2}\right]$.

\subsubsection{Autolinear}

A mapping is chosen such that $x=0$ always maps to $y=0.5$, to ensure a mid-grey representation of zero. The other $(\mathrm{x}, \mathrm{y})$ pair is selected based upon the min and max of the input set. The largest magnitude in $x$ is mapped to $y= \pm 1$, preserving sign. To wit,

$$
\begin{gathered}
x_{1}=0 \rightarrow y_{1}=0.5 \\
x_{2}=|x|_{\text {max }} \rightarrow y_{2}=\operatorname{sgn}\left(x_{m}\right), \quad \text { where } m \text { is taken from }|x|_{\text {max }}
\end{gathered}
$$




\subsubsection{Min-Max}

Min-Max maps the full dynamic range to fit within the full displayable dynamic range:

$$
[\min (x), \max (x)] \rightarrow[0,1]
$$

\subsubsection{Z-Score}

From statistics, the z-score is the normalized representation of a value, having zero-mean and unity variance. And input value is mean-subtracted, then divided by the input set's standard deviation, as thus denoted:

$$
y=\frac{x-\operatorname{mean}(x)}{\sigma_{x}}
$$

\subsection{Nonlinear}

\subsubsection{Gamma Mapping (Power Law)}

The power law, also known as gamma correction, or gamma mapping, is a nonlinear mapping that may be tuned to place emphasis on smaller values or larger values within the input range. Gamma correction used in this thesis is parameterized by two values: $\gamma$ determines the shape (emphasis) and $x_{\text {sat }}$ determines the width. $x_{\text {sat }}$ may be chosen as a percentile, such that values greater than that percentile will be mapped to 1 . Equation 12 defines the gamma map, and Figure 24 shows an example of a uniform random distribution of 1000 points between 1 and 100 . Both the transfer function and the input and output histograms are shown. In this example, $\gamma=$ 0.5 , and $x_{\max }=98$, or the $98^{\text {th }}$ percentile.

$$
y=\left\{\begin{array}{ccrl}
-1 ; & x & <x_{\text {sat }} \\
\frac{\operatorname{sgn}(x) \cdot|x|^{\gamma}}{x_{s a t}{ }^{\gamma}} ; & -x_{s a t} & \leq x \leq x_{\text {sat }} \\
1 ; & & x>x_{\text {sat }}
\end{array}\right\}
$$




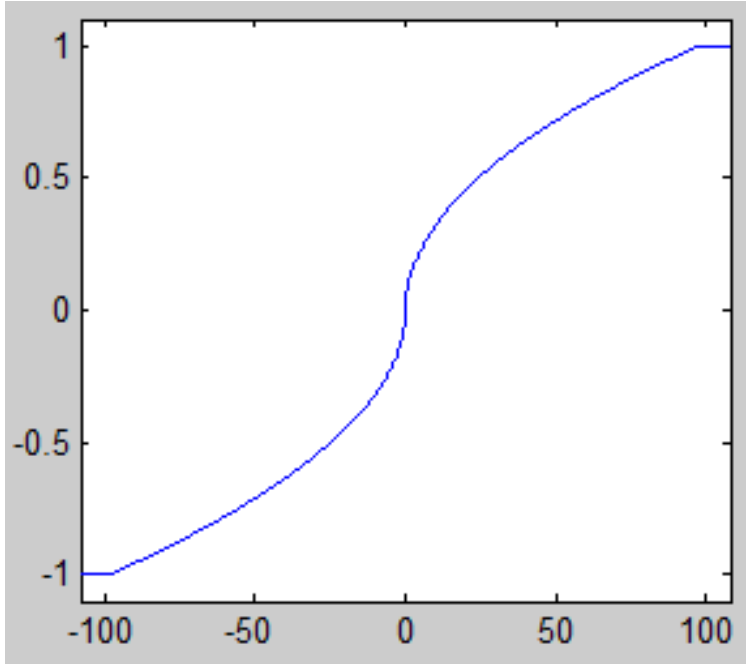

(a) Transfer Function

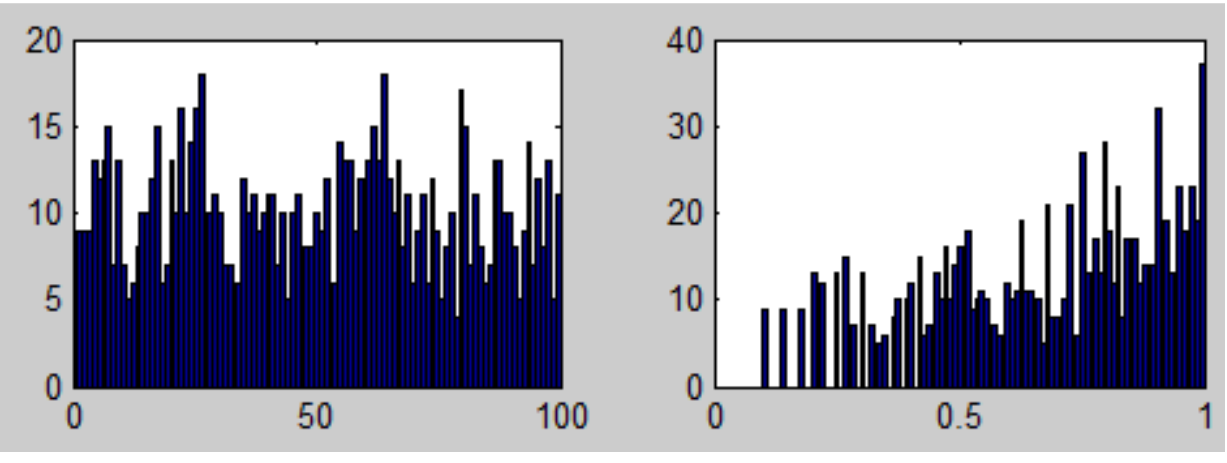

(a) Example Input Distribution (b) Corresponding Output Distribution $\left(\gamma=0.5, x_{\max }=98\right)$

Figure 24 - Gamma Mapping Example Transfer Function and Histograms

\subsection{Composite}

\subsubsection{Z-Gamma}

In Section 5.5, both the linear z-score and the nonlinear gamma mapping will be combined, in that order. The composite z-score and gamma mapping mapping will be referred to as "z-gamma", or, in the code, as zgamma.

\subsubsection{ZA-Gamma}

Same as Z-Gamma, except that the gamma mapping acts on the magnitude of the Zscore. 


\subsection{Intensity Mapping Function Summary}

Table 13 - Intensity Mapping Function Descriptions

\begin{tabular}{|c|c|c|}
\hline \multirow[t]{2}{*}{ Function / Script Name } & \multicolumn{2}{|c|}{ Description Overview } \\
\hline & Function Input & Function Output \\
\hline \multirow[t]{2}{*}{ linear $4 \mathrm{~m}$} & $\begin{array}{l}\text { Mode 1: Linear mapping } \\
\text { parameters. }\end{array}$ & $\begin{array}{l}\text { Mode 1: } x 4 \text { mStruct that fully } \\
\text { specifies the mapping. }\end{array}$ \\
\hline & $\begin{array}{l}\text { Mode 2: } x 4 \mathrm{mStruct} \text { and array } \\
\quad \text { of intensities. }\end{array}$ & $\begin{array}{l}\text { Mode 2: Data mapped according } \\
\text { to } x 4 \mathrm{mStruct} \text {. }\end{array}$ \\
\hline linearx4mlim & Input range and output range & $\begin{array}{l}\text { Linear mapping parameters that } \\
\text { enforce the specified range. }\end{array}$ \\
\hline \multirow[t]{2}{*}{ nonlinMap } & $\begin{array}{l}\text { Mode 1: Nonlinear mapping } \\
\text { parameters. }\end{array}$ & $\begin{array}{l}\text { Mode } 1: \mathrm{x} 4 \mathrm{mStruct} \text { that fully } \\
\text { specifies the mapping. }\end{array}$ \\
\hline & $\begin{array}{l}\text { Mode 2: } x 4 \mathrm{mStruct} \text { and array } \\
\text { of intensities. }\end{array}$ & $\begin{array}{l}\text { Mode 2: Data mapped according } \\
\text { to } \mathrm{x} 4 \mathrm{mStruct} \text {. }\end{array}$ \\
\hline
\end{tabular}

\section{CLASSIFIERS}

Classification systems combine one or more feature frames to produce a classification frame. A classification frame is a binary image, the pixels of which correspond to the same pixels as in a feature image, but represent a decision on whether the pixel represents heat or not heat. The systems described here have some merit on their own at the onset, then will be analyzed, tuned, expanded, and improved. Common metrics will evaluate classifiers on their performance, and guide the line of inquiry in gainful directions.

\subsection{Metrics}

A standardized test will apply uniformly for all classifiers under study. For many cases, the testing will apply repeatedly to compare different classification parameters, such as a window size or a time constant. Error rate serves as primary metric, and computation time the secondary. 


\subsubsection{Error Rate}

The primary metric for judging the effectiveness of a detection system is its error rate. Error rate is defined as:

$$
e=\frac{t p}{t p+f p}
$$

where $t p$ represents true positives, and $f p$ false positives. In literature, this is known as "precision." The measure of truth is whether a positive falls within a prescribed heat region. This measure only approximates the real truth. It would require more accurate knowledge of truth (that is, the exact location and boundary) of the heat plume at all times) to count true negatives ( $t n)$ and false negatives $(f n)$, so they will be ignored.

\subsubsection{Categorized Detection Count (CDC)}

In addition to an error percentage, the positives will be assigned to groups including heat region and common problem areas: pedestrian region, car region, and noise region. Counting these groups gives more information on the ability of each system to reject different types of noise. Figure 25(a) shows these masks as white, enclosed by the colored bands to identify them. (b) shows the heat mask and the non-heat mask. These masks were created by opening a frame such as variance in MS Paint, and hand-drawing the region around it. The heat mask was fine tuned by subtracting (in Matlab) a horizontal line where interface noise lived. 


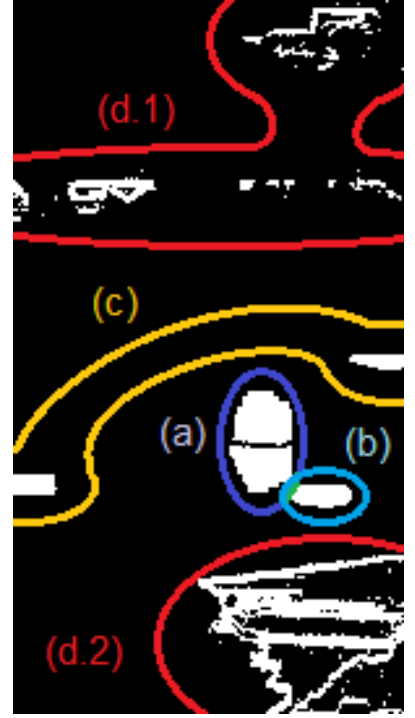

(a)
a: Heat region
b: Pedestrian region
c: Car region
d: Noise, near and far
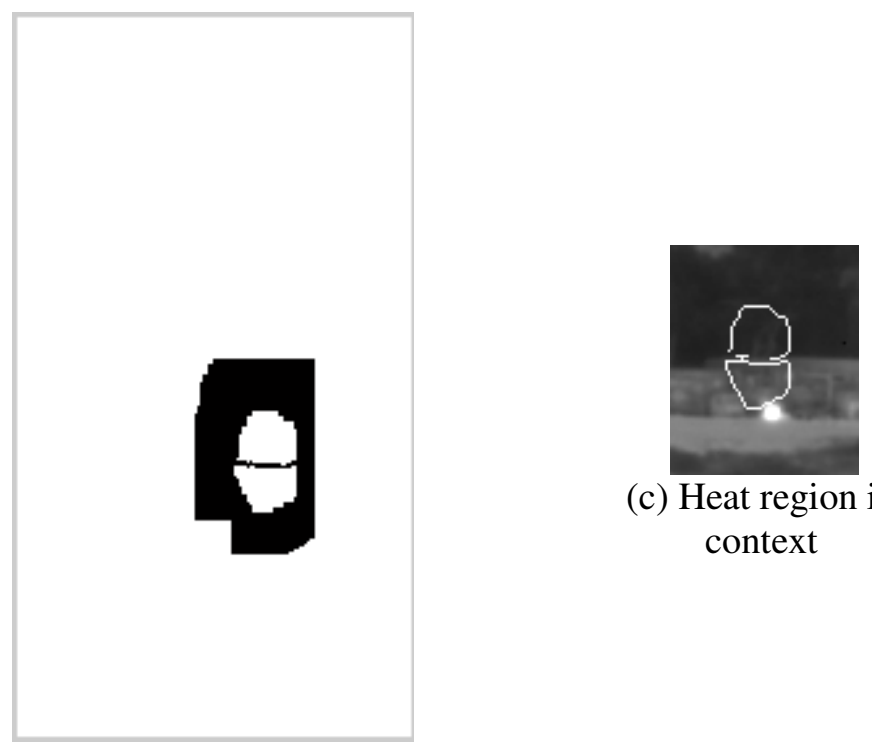

(c) Heat region in context

Figure 25 - Movement Category Masks

Error counters will count the sum of heat-assigned pixels that fall within the specified masks over the standardized frame range of video: $510-5000$.

\subsubsection{Computation Time}

The code is structured to run one operation at a time though a full video in a completely scalable fashion. The code doesn't have a direct computation time, as eachbut this metric will estimate the frame rate supported by the algorithm by dividing the number of standardized frames $(5000-510=4490)$ by the total computational time, resulting in a frame rate (fps).

\subsection{Variance Corridor / Bayesian Classifier}

\subsubsection{Rationale}

The rationale for the variance corridor is simply that the motion of the heat plume may reside within a specific range of variances that notch thresholding can reveal. 
The rationale for the Bayesian classifier is that every type of motion might be characterized by its time-variance. In a general sense, Bayesian classification relies on a-priori knowledge, and often describes Gaussian data. The data considered here aren't Gaussian distributed, but the concept doesn't differ.

\subsubsection{Experiment}

Recall from Section 3.1.2.3 that sequences of length 30 were extracted over a range of 220 frames, beneath the masks shown in Figure 18, resulting in hundreds and thousands of variance samples per mask. These variances were statistically described by Table 8 .

Now consider Figure 26, in which the variances are binned with exponential centers. The hypothesis: that a pixel may be classified to one of the seven classes shown in Figure 26 by the following method:

1. Compute its variance value over a sequence of 30 , just as the histogram data.

2. Determining which bin it falls into.

3. Identify which class has the largest corresponding bin (Nearest neighbor), and assign the pixel to that class. 

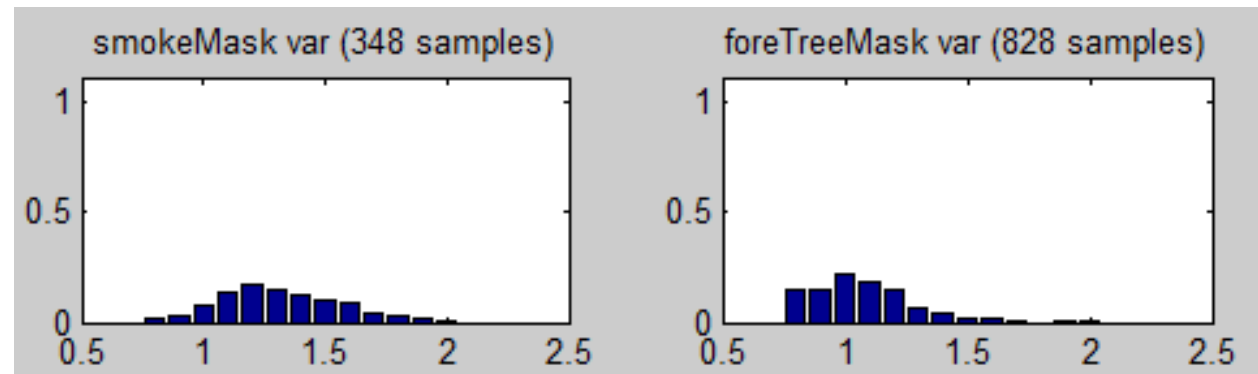

Bin

centers:

6.3096

7.9433

10.0000

12.5893

15.8489

19.9526

midTree1Mask var (714 samples)

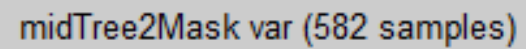

25.1189
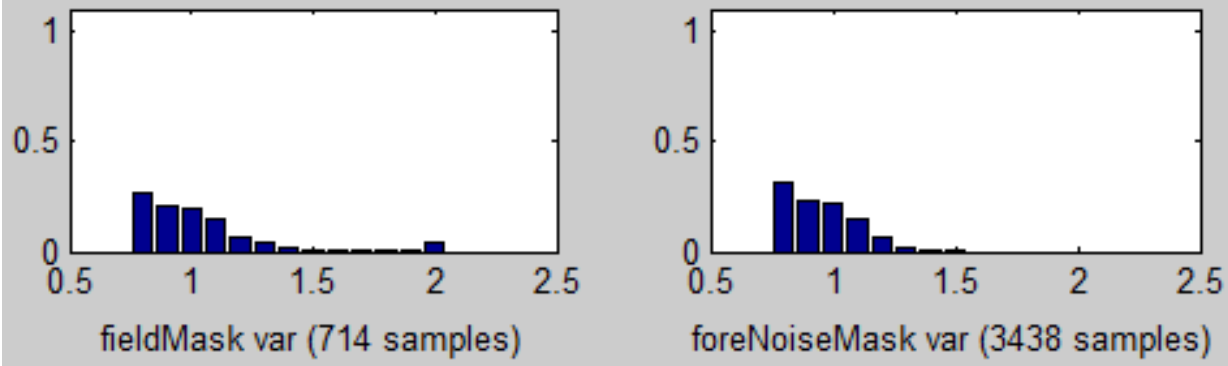

31.6228

39.8107

50.1187

63.0957

79.4328

100.0000

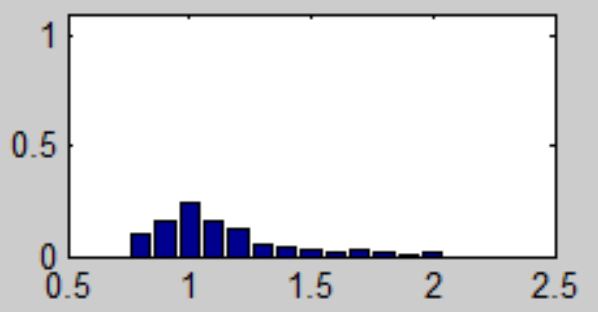

foreNoiseMask var (3438 samples)

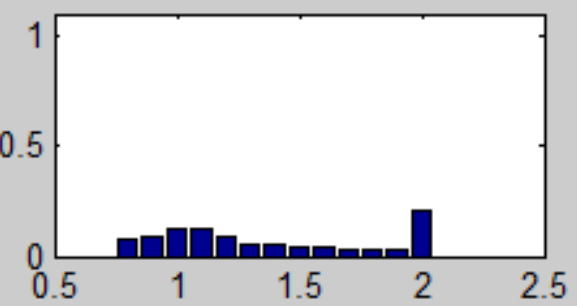

farNoiseMask var (2802 samples)
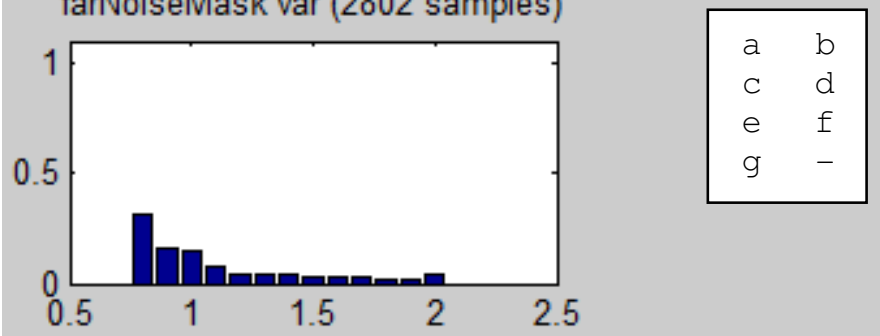

Figure 26 - Exponentially Binned Variance Histogram Per Masked Region (exponentially indexed abscissa: $10^{\wedge} \mathrm{x}$ )
(a) Heat plume (a.k.a. smoke plume)
(b) Near foliage
(c) Mid-ground foliage 1
(d) Mid-ground foliage 2
(e) Grassy field
(f) Near noise
(g) Distant noise 
From this method, a mapping between variance and class can be established: Table 14 shows the mapping.

Table 14 - Correspondence Between Bin Center and Class with Largest Occurrence

\begin{tabular}{|c|c|c|c|c|c|c|c|c|c|c|c|c|c|}
\hline $\begin{array}{c}\text { Linear } \\
\text { Bin Center }\end{array}$ & 6.3 & 7.9 & 10 & 12 & 15 & 20 & 25 & 31 & 39 & 50 & 63 & 79 & 100 \\
\hline $\begin{array}{c}\text { Exponential } \\
\text { Bin Center }\end{array}$ & 0.8 & 0.9 & 1.0 & 1.1 & 1.2 & 1.3 & 1.4 & 1.5 & 1.6 & 1.7 & 1.8 & 1.9 & 2.0 \\
\hline Class & $\mathrm{d}$ & $\mathrm{d}$ & $\mathrm{e}$ & $\mathrm{b}$ & $\mathrm{a}$ & $\mathrm{a}$ & $\mathrm{a}$ & $\mathrm{a}$ & $\mathrm{a}$ & $\mathrm{a}$ & $\mathrm{f}$ & $\mathrm{f}$ & $\mathrm{f}$ \\
\hline
\end{tabular}

The result of the Bayesian variance classifier is simply a variance corridor in the range of 14.2 < var $<56.6$ (thresholds bisect adjacent linear bin centers). Figure 27 shows the result. It actually appears to select not smoke near the heat plume.

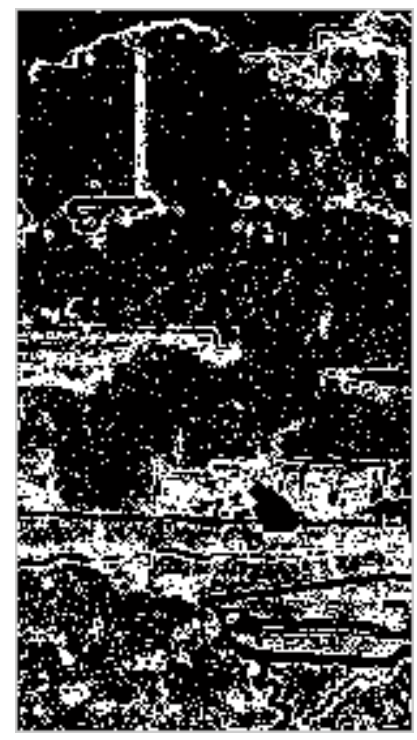

Figure 27 - Result of Variance Corridor / Bayesian Variance Classification (oats1, frame 1799) 


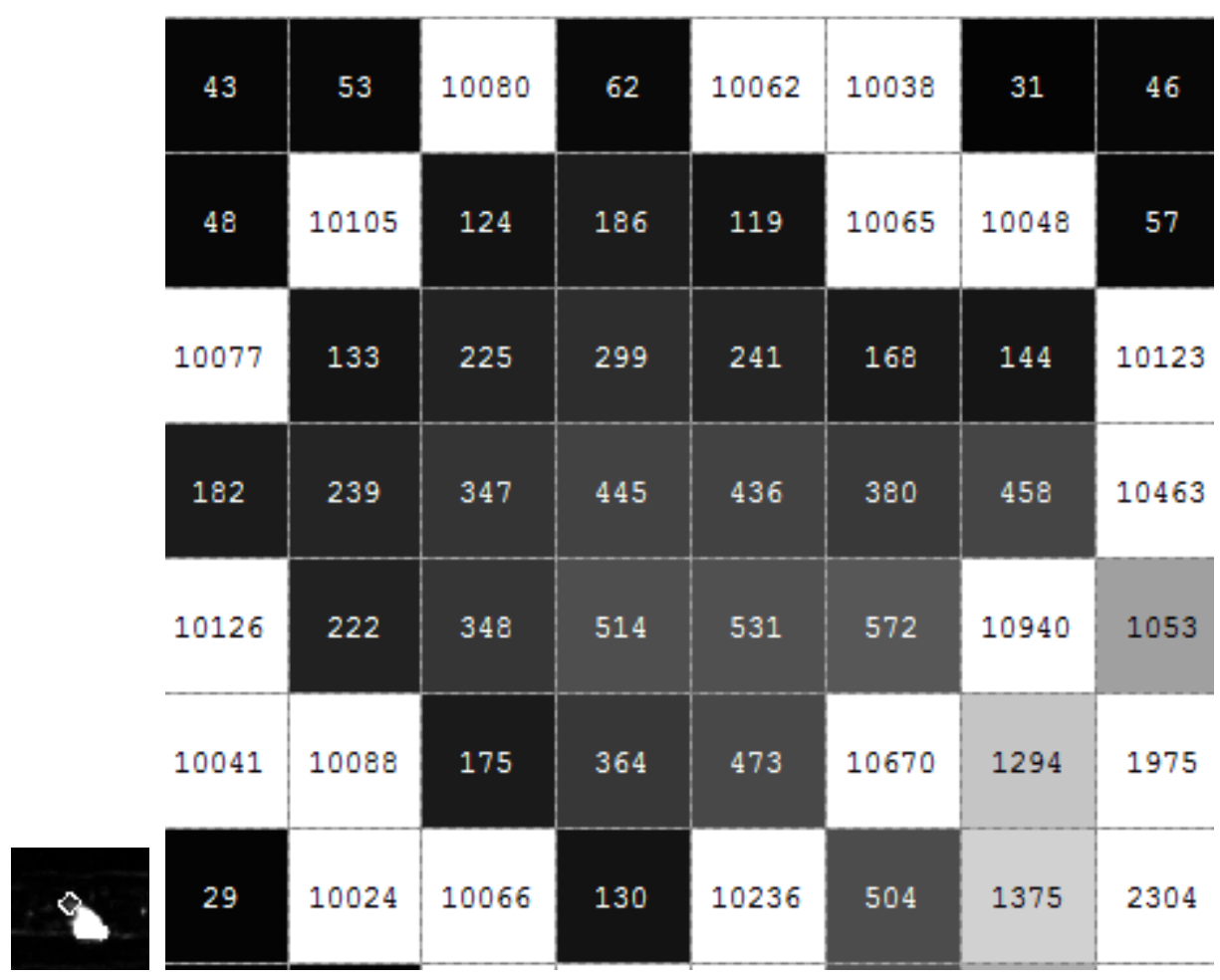

(a)

(b)

Figure 28 - Variance within Heat Plume of Figure 27

(a) Variance image with an edge added to the heat plume area

(b) Blow-up of heat plume area (ignore the edge pixels, identified by intensity $\sim 10,000$ )

Figure 28 shows that the heat plume actually resides in an approximate variance range of $[100,500]$, far from the range that this implementation of a Bayesian classifier.

\subsubsection{Conclusion}

Bayesian classification based solely on variance detects motion indiscriminately, passing far more than just the heat plume, but this exploration did reveal where the heat plume lives: in the range $[100,500]$. This knowledge will prove useful in Section 5.4 as a filter component.

Since this classification system fails so completely, metrics are considered superfluous.

\subsection{Variance Set Statistics - Mean and Max Thresholding (MMT)}

By inspection of a feature frame while sliding its dynamic range, a range of values can be found wherein the heat plume is present. Any one feature is unlikely to perfectly identify the heat 
plume, non-inclusive of other background content, but combining thresholds of multiple features can provide more accurate classification.

\subsubsection{Rationale}

Recall from exploration of the variance set statistics in Section 3.1.2.3 that the mean and max statistics appeared to discriminate between classes (heat plume, foliage, etc). Inspection of the numbers alone justifies this classification experiment. An intuitive justification: The mean of the variance set represents how much a pixel varies long-term, a measure of persistence relative to the set size (a defining parameter), while the max represents the greatest or fastest change, which should be high at the onset of growth (when a heat plume begins to pass through a pixel). Heat plume tends to be sporadic, varying noticeably (between 100 and 500) where it touches, but not always touching the same location. Hence the typical VSS mean would be less than [100, 500] while the typical VSS max would be near the top of $[100,500]$. The hypothesis is that other entities within the scene sufficiently differ to distinguish them from the heat plume.

\subsubsection{Experiment}

Frames of the same time index were opened in varMean and varMax (oats1, stabilized, sequence length 30, set size 20, frame 3140) to investigate the values in the heat plume. Figure 29 shows the two frames (in the region-of-interest already shown in Figure 1) contrast adjusted for clarity to the same range of $[0,800]$. Note that varMax is sharper and brighter than varMean, as expected. The heat plume in varMax shows up very strong and large. The border of the trees with the hot building wall (refer back to Figure 1 if necessary) also shows up very strong. This will prove challenging to discriminate from heat plume. Figure 30 shows a zoomed-in view of the heat plume, also contrast adjusted for clarity, and it shows the pixel intensities. The background intensity near the barbecue pit generally hovers between 40 and 100, where the heat plume varies wildly between 100 and beyond saturation at 65535, consistent with findings in Section 5.2.2. 


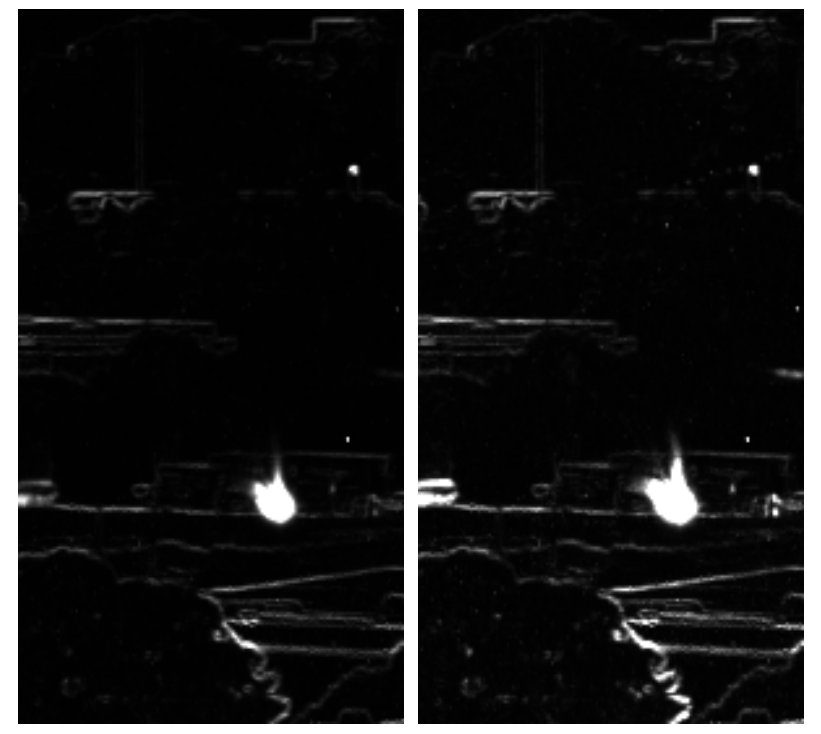

$\begin{array}{ll}\text { (a) varMean } & \text { (b) varMax }\end{array}$

Figure 29 - varMean and varMax from oats1, Frame 3140 (Contrast adjusted for clarity to the same range: $[0,800])$

\begin{tabular}{|l|l|l|l|l|l|l|l|l|l|l|l}
\hline 47 & 72 & 52 & 52 & 83 & 84 & 92 & 123 & 179 & 509 & 933 & 613 \\
\hline 114 & 135 & 139 & 111 & 109 & 170 & 211 & 207 & 251 & 681 & 1121 & 1008 \\
\hline 193 & 304 & 428 & 411 & 259 & 248 & 374 & 426 & 479 & 1004 & 2157 & 1785 \\
\hline 249 & 463 & 781 & 959 & 672 & 386 & 495 & 766 & 944 & 1646 & 3768 & 3240 \\
\hline 281 & 509 & 930 & 1192 & 1351 & 855 & 686 & 987 & 1596 & 2956 & 6609 & 5984 \\
\hline 340 & 563 & 1047 & 1672 & 2407 & 2087 & 1354 & 1331 & 2642 & 5751 & 12488 & 11784 \\
\hline 338 & 525 & 867 & 1571 & 2728 & 3376 & 2855 & 2628 & 4903 & 11915 & 25961 & 26867 \\
\hline 253 & 369 & 607 & 1030 & 1947 & 3523 & 5102 & 5611 & 10911 & 29566 & 60787 & 64768 \\
\hline 294 & 585 & 1106 & 1902 & 4433 & 12199 & 19683 & 28249 & 65535 & 65535 & 65535 \\
\hline
\end{tabular}

Figure 30 - varMean in the Heat Plume, Frame 3140 
The precise variance threshold of where heat plume starts and ends is subjective. Figure 31 shows varMean at several threshold values to give an idea of where its histogram is distributed.

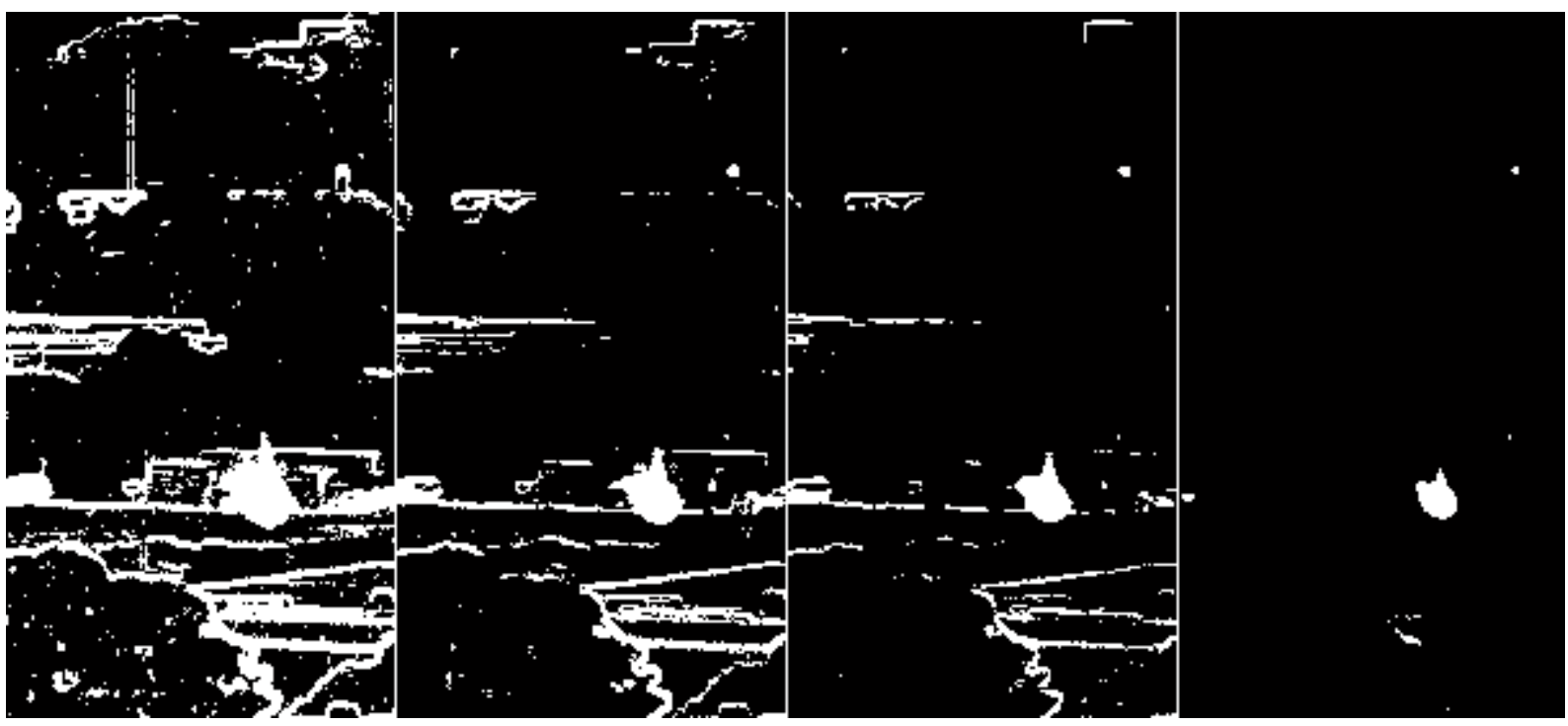
(a) varMean $>20$
(b) varMean $>50$
(c) varMean $>100$
(d) varMean $>500$

Figure 31 - varMean at Various Thresholds

Three sets of thresholds were selected subjectively. The Loose thresholds show more heat plume signal while letting in much background noise as well. The Tight thresholds focus more around the observed ranges of heat plume, so reject more noise, yet also detect a fewer true heat pixels. Config 3 was based on early explorations. The same process was repeated for varMax, and the threshold values selected to represent the range of heat plume are shown in Table 15. See Figure 32 for realizations of these thresholds.

Table 15 - Variance Set Statistics Thresholds (sequence length 30, set size 20)

\begin{tabular}{llll}
\hline & Loose & Tight & Config 3 \\
\hline varMean & {$[40,700]$} & {$[10,100]$} & {$[60,150]$} \\
$\operatorname{varMax}$ & {$[60,5000]$} & {$[100,600]$} & {$[500,1500]$} \\
\hline
\end{tabular}




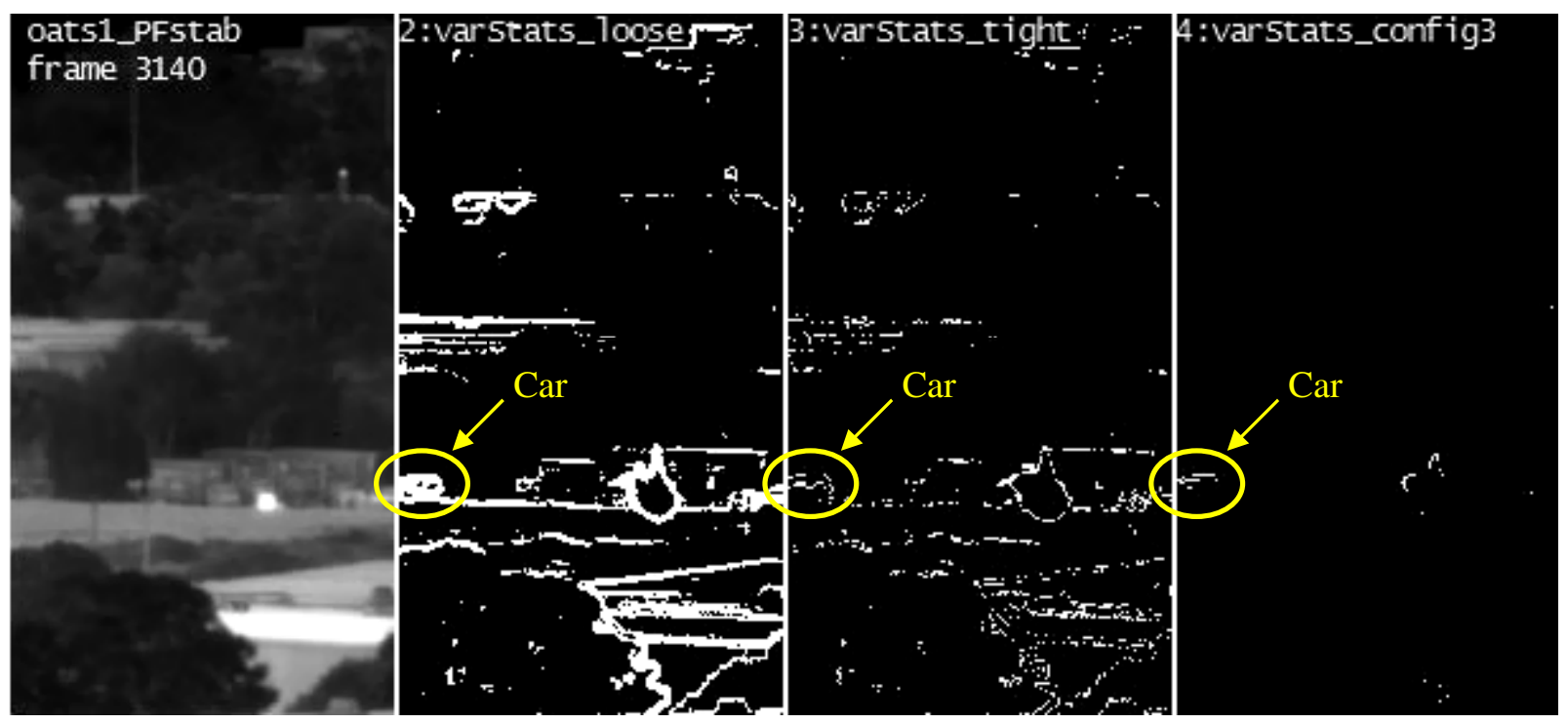

Figure 32 - Intersection of Thresholded VSS Mean and Max for Different Configurations (threshold values)

Hardly any true signal survives the Tight and Config3 configurations, so the focus will rest with the loose configuration. To clean up the noisy information, temporal signature expectations can be applied. Recall the growth property of the heat plume (by its association with fire, the originator of these characteristic properties). The static background noise has movement that migrates very little. These can be eliminated by discounting the pixels that stay asserted for $\mathrm{N}$ consecutive time samples. The "VSL-isAllN" signals in Figure 33 mean "varStats_Loose AND NOT isAllN", where the isAllN frame's pixels are true if the corresponding varStats_loose pixels are true for the past $\mathrm{N}$ frames (the varStats_loose video has time resolution of $3 \mathrm{fps}$ ). They are also median filtered with $3 \times 3$ and $5 \times 5$ neighborhoods for comparison.

Figure 34 shows the categorized detection count per VSL channel for several filter lengths and median filter sizes. 

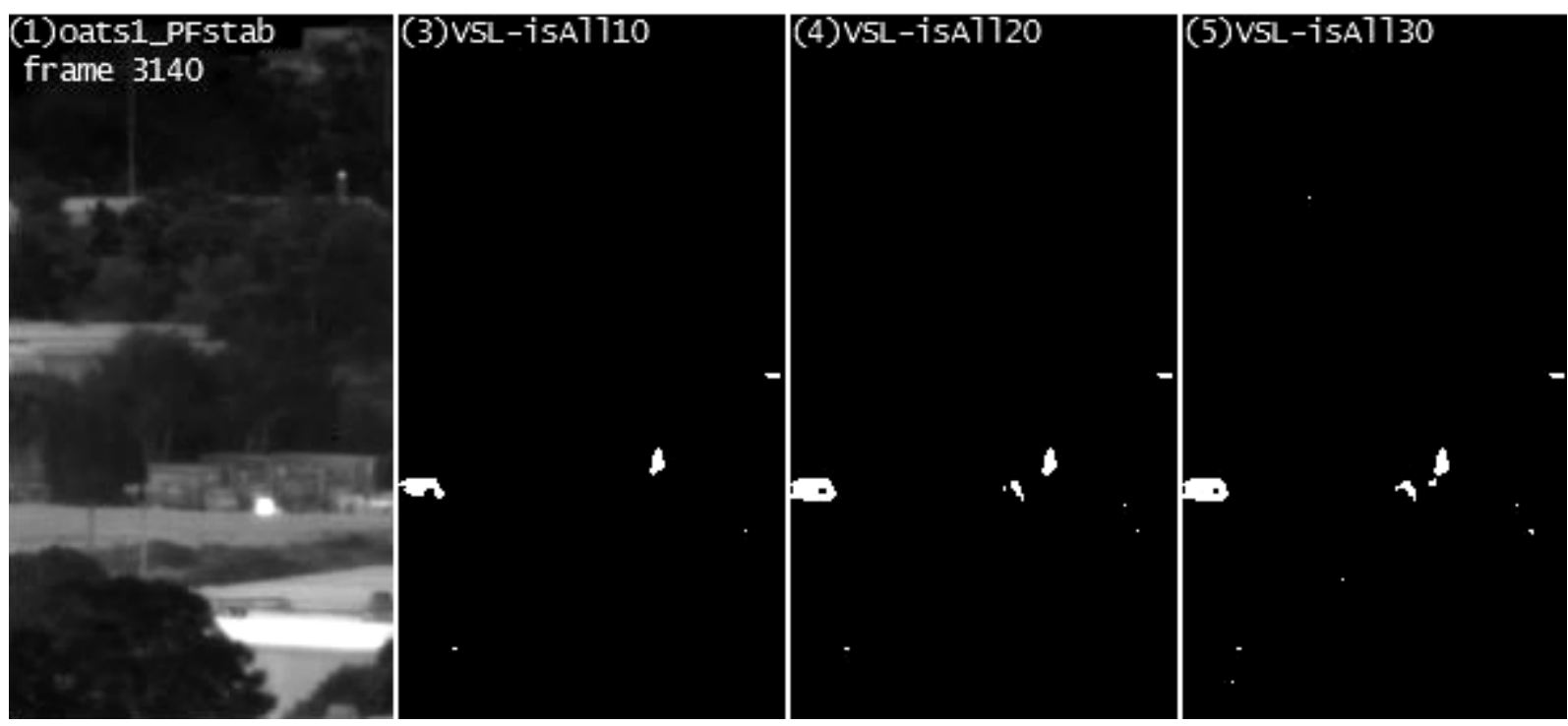

Figure 33 - Comparison of Filter Lengths on varStats_loose Configuration (Demo: varStats_loose_isAll123_3fps.avi)

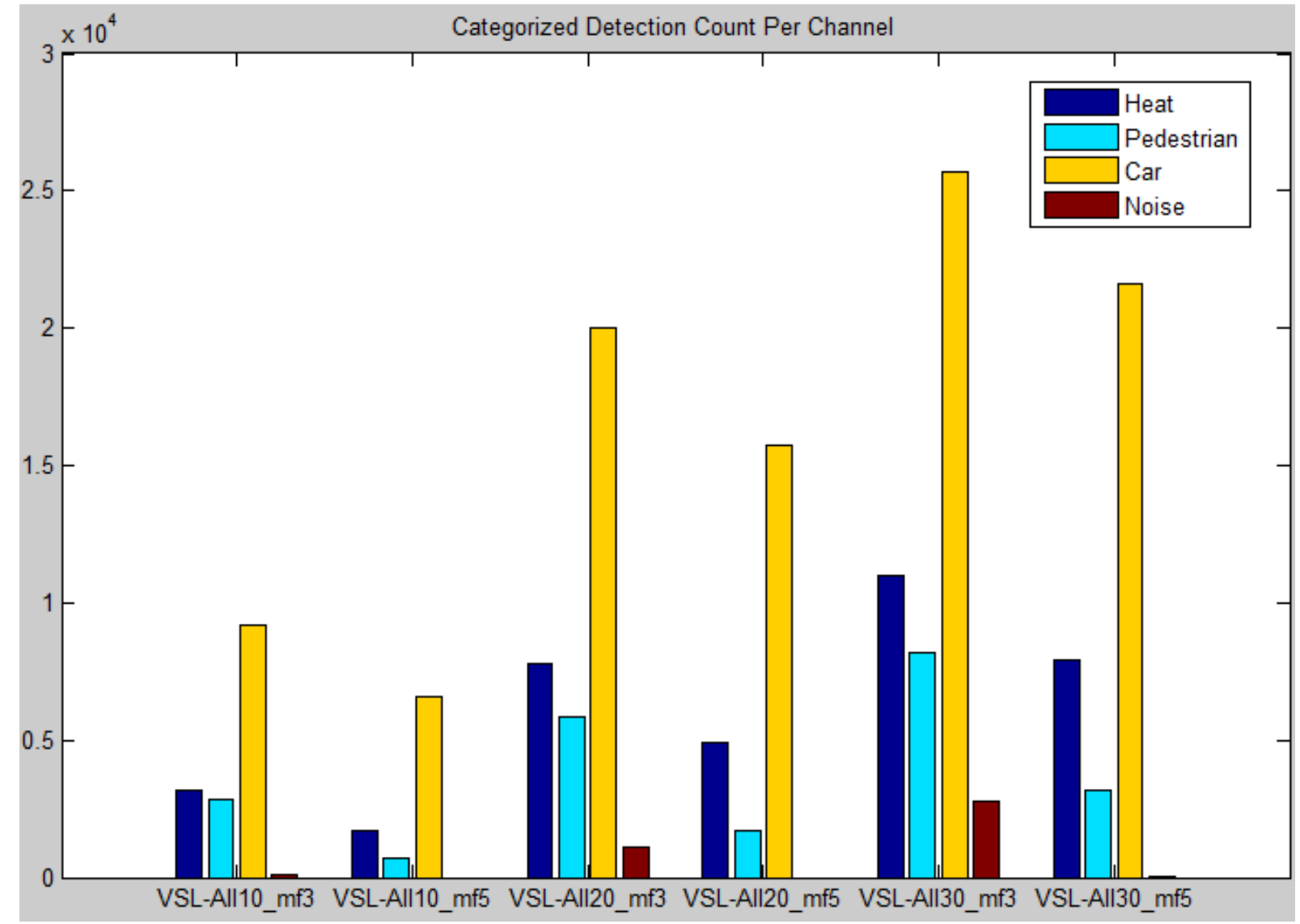

Figure 34 - Categorized Detection Count Per Channel of VSL for Several Filter Configurations 
Table 16 - Error Analysis Over Filter Configuration

$\begin{array}{llll}\text { Channel name: } & \text { Good } & \text { Bad } & \text { E_avg } \\ \text { varStats_loose_-_isAll10_medfilt3 } & 3206 & 15710 & 0.727 \\ \text { varStats_loose_-_isAll10_medfilt5 } & 1697 & 8211 & 0.482 \\ \text { varStats_loose_-_isAll20_medfilt3 } & 7778 & 40787 & 0.827 \\ \text { varStats_loose_-_isAll20_medfilt5 } & 4891 & 20891 & 0.750 \\ \text { varStats_loose_-_isAll30_medfilt3 } & 11017 & 62279 & 0.842 \\ \text { varStats_loose_-_isAll30_medfilt5 } & 7891 & 32077 & 0.785\end{array}$

Table 17 - Computation Time for MMT

\begin{tabular}{ll}
\hline Process & Time (s) \\
\hline Variance Set Statistics calculation & $550 \mathrm{~s}$ \\
VarStats_Loose classification & $25 \mathrm{~s}$ \\
isAll computation & $25 \mathrm{~s}$ \\
Binary subtraction and median filtering & $41 \mathrm{~s}$ \\
Total Time: & $640 \mathrm{~s}$ \\
\hline Capable Frame Rate: & 4490 frames / $640 \mathrm{~s}=\mathbf{7 . 0} \mathbf{f p s}$ \\
\hline
\end{tabular}

\subsubsection{Conclusion}

Simply taking the intersection of the thresholded mean and max variance set statistics did not effectively differentiate between heat plume and the interface noise and the moving vehicles. The Loose configuration had a strong heat plume signal as well as pervasive noise, while the Tight configuration selected little of both, and the Config 3 barely selected heat at its most intense. The method required a noise rejection stage before it could offer any use. Implementing the "isAll" filter removed the static noise, vastly cutting down on tree and interface noise.

The best configuration involved subtracting a shorter filter (length 10), then median filtering with a $5 \times 5$ neighborhood. 


\subsection{Variance Set Statistics - Mean by Median (MBM) Quotient}

\subsubsection{Rationale}

Intuitively, the quotient of mean by median will detect outlier changes, which describes the heat plume when it wanders through a pixel irregularly (an example of the growth property). The simple reason is that the mean is more sensitive to outliers than the median.

This section will cursorily explore other quotient features aimed at reducing unwanted background noise. The DRMAD (introduced in Section 3.1.5) still has some noise in it, which could be reduced if it were scaled down in proportion to the known movement. The DRMAD as well as the original measurement will be divided by the standard deviation per pixel.

\subsubsection{Experiment}

Figure 35 shows the operations and results. The original measurement (denoted "src" in the figure), even when divided by the standard deviation, does not offer a way to differentiate between the heat plume and the noise, which still is prominent. The DRMAD/standard deviation quotient yields much noise with no clear indicator of the heat plume. However, the VSS max/mean quotient shows a very clear signal, as does the max/mean quotient. The max/mean quotient corroborates the concept of the VSS max less mean, developed in Section 5.3. 


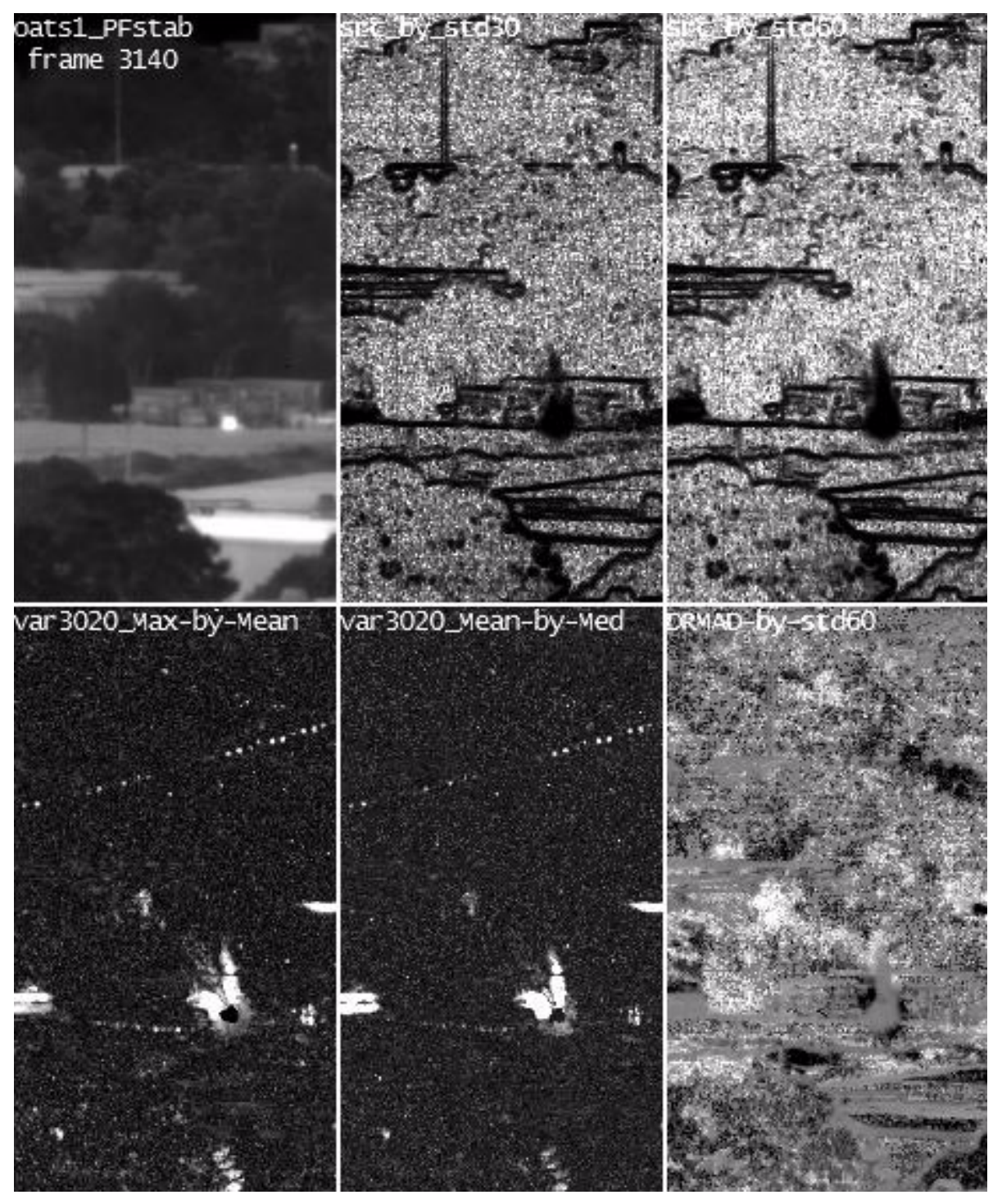

Figure 35 - Comparison of Feature Quotients

The VSS parameters (sequence length and set size) are varied in an attempt to identify optimal values based on a calculated error rate (defined in Section 5.1). The error calculation requires a classification (binary) image, so the feature images of Figure 36 will be thresholded, then median filtered to reduce noise. Figure 37 shows the MBM frames after a threshold an empirical threshold ( $\geq 3)$ and a $5 \times 5$ median filter. 


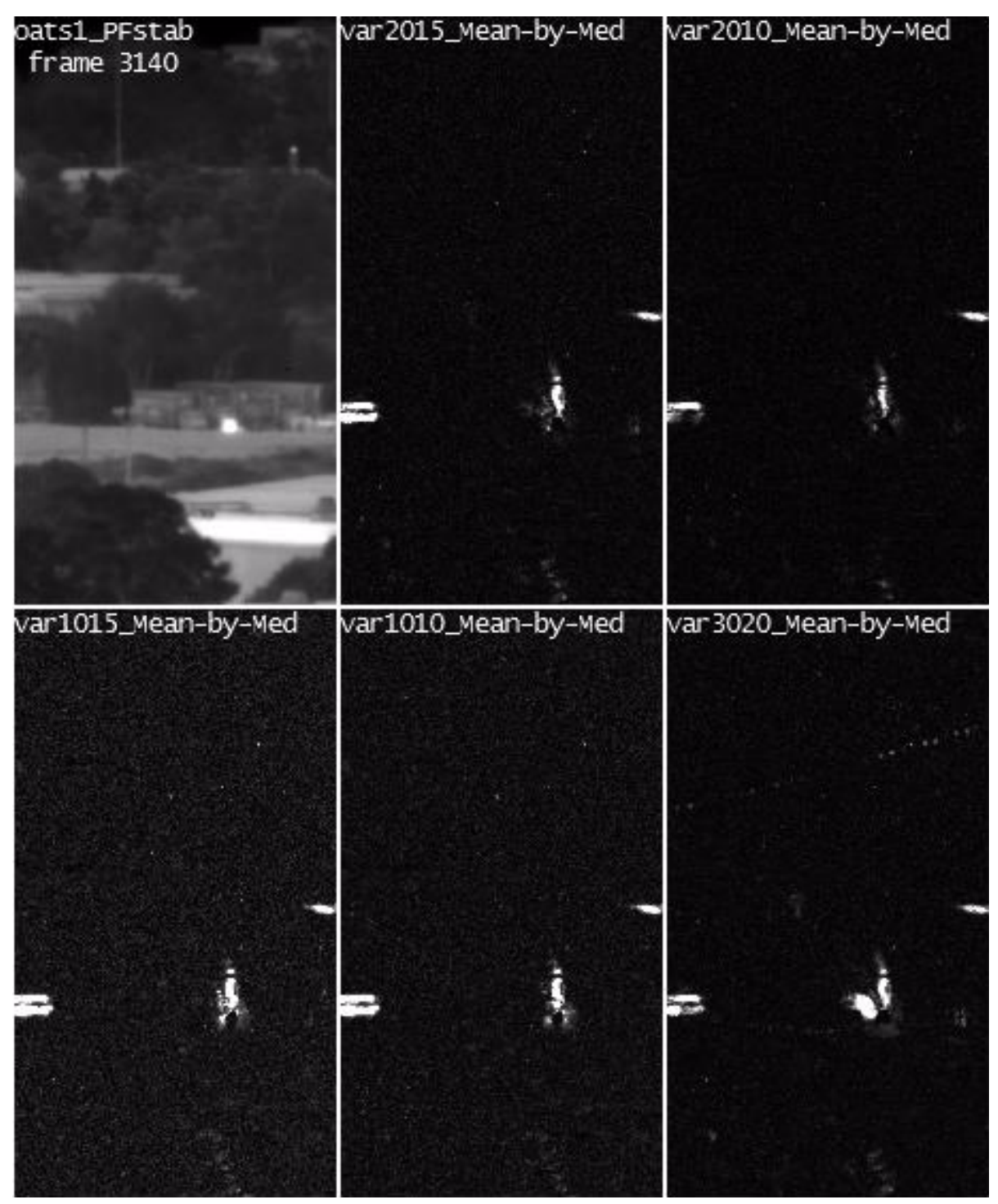

Figure 36 - Comparison of VSS parameters on MBM 


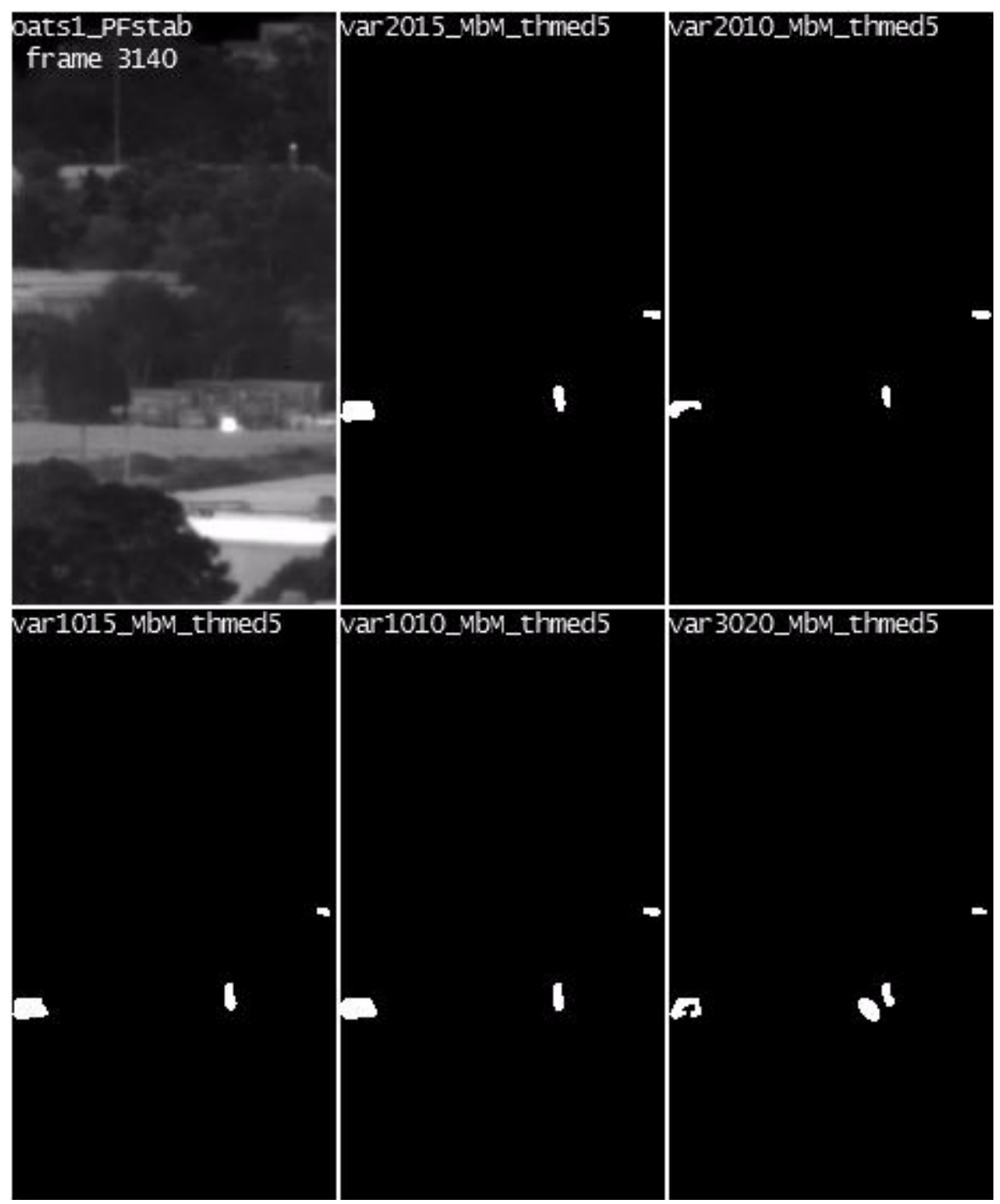

Figure 37 - Comparison of VSS Parameters on MBM, Thresholded, and Median Filtered

Table 18 shows the error rates of the thresholded and median filtered MBM frames. $\operatorname{Var}(\mathrm{XX})(\mathrm{YY})$ refers to sequence length $\mathrm{XX}$ and set size $\mathrm{YY}$. The error rate proves more sensitive to the set size than the sequence length.

Table 18 - Error Rates of Figure 37 Frames

$\begin{array}{llll}\text { Channel name: } & \text { Good } & \text { Bad } & \text { E_avg } \\ \text { var2015_Mean-by-Med_thmed5 } & 5152 & 42409 & 0.794 \\ \text { var2010_Mean-by-Med_thmed5 } & 2980 & 31555 & 0.695 \\ \text { var1015_Mean-by-Med_thmed5 } & 4602 & 39879 & 0.743 \\ \text { var1010_Mean-by-Med_thmed5 } & 2936 & 32175 & 0.680 \\ \text { var3020_Mean-by-Med_thmed5 } & 6365 & 49999 & 0.811\end{array}$




\subsubsection{Variance Augmented Mean-By-Median Quotient (VAMBM)}

The birds, cars, and pedestrians still show up in MBM alone, but when intersected with the 30-length variance (referred to as "var30" in the pane captions) thresholded above 100, the birds disappear, while the heat plume remains, as was found in Section 3.1.1.3. Figure 38 shows this operation.

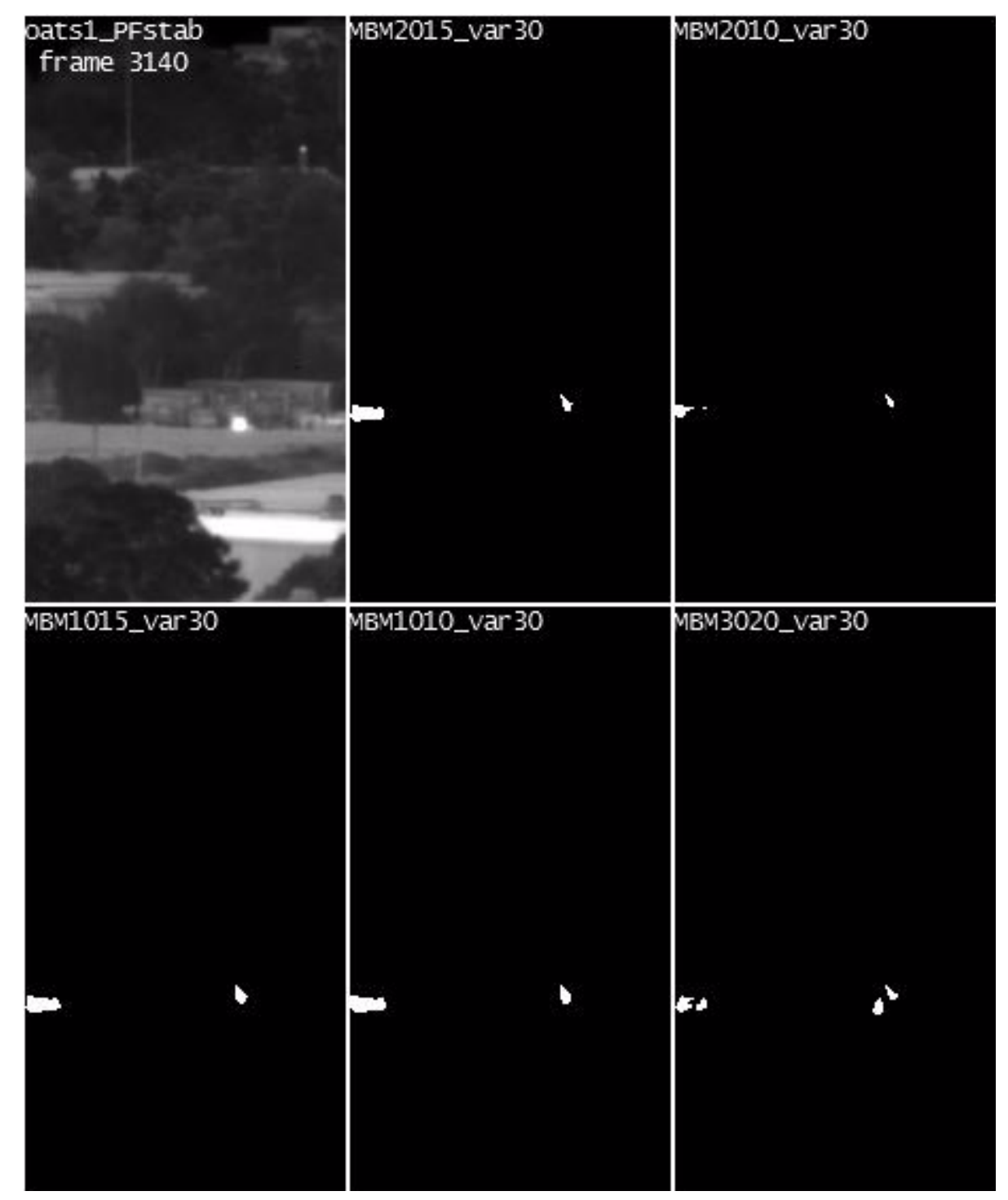

Figure 38 - Comparison of VSS Parameters for VAMBM Data (variance length 30)

Let the intersection of MBM with var30 be referred to as the MBM_var30 data, or VAMBM classification. Figure 39 shows the categorized detection count. The "Noise" refers to 
foliage and interface noise. The abscissa labels refer to MBM_var30 series' but are shortened for display purposes.

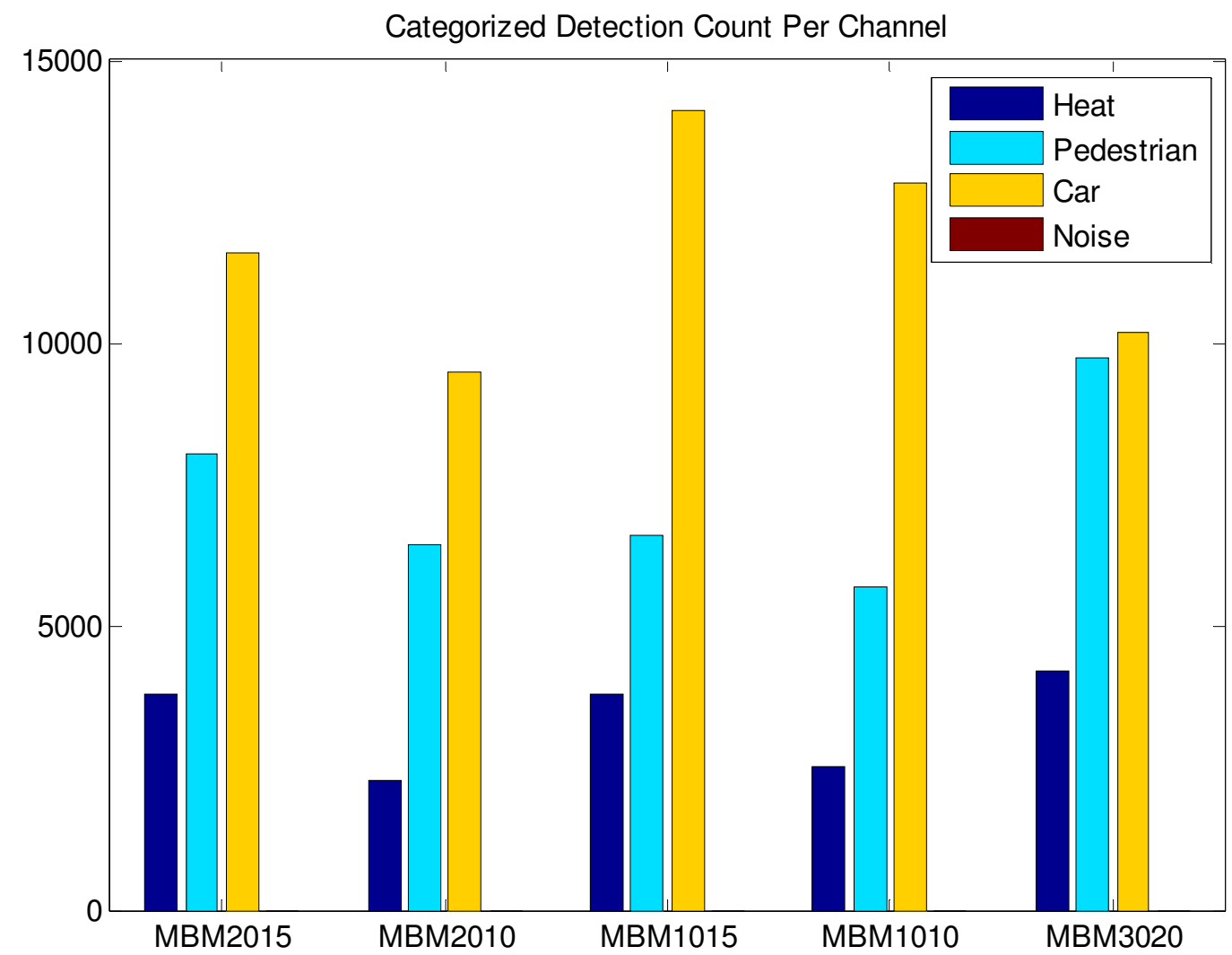

Figure 39 - Categorized Detection Count Per Channel of MBM_var30 (VAMBM) for Several VSS Configurations

Table 19 - Error Rate of MBM_var30 Over VSS Configurations

$\begin{array}{llll}\text { Channel name: } & \text { Heat } & \text { Not } & \text { E_avg } \\ \text { MBM2015_var30 } & 3815 & 21289 & 0.739 \\ \text { MBM2010_var30 } & 2299 & 17211 & 0.650 \\ \text { MBM1015_var30 } & 3814 & 21308 & 0.675 \\ \text { MBM1010_var30 } & 2530 & 19355 & 0.624 \\ \text { MBM3020_var30 } & 4217 & 23031 & 0.766\end{array}$


Table 20 - Computation Time of VAMBM

\begin{tabular}{ll}
\hline Process & Time (s) \\
\hline Variance Set Statistics calculation & $550 \mathrm{~s}$ \\
Quotient calculation & $185 \mathrm{~s}$ \\
Threshold and median filter & $16 \mathrm{~s}$ \\
Dilate and threshold variance, take intersection & $28 \mathrm{~s}$ \\
Total Time: & $779 \mathrm{~s}$ \\
\hline Capable Frame Rate: & 4490 frames $/ 779 \mathrm{~s}=\mathbf{5 . 7} \mathbf{f p s}$ \\
\hline
\end{tabular}

\subsubsection{Conclusion}

MBM effectively filters out the trees by passing outlier movement that the foliage is too stationary to exhibit. Figure 36 shows the MBM before thresholding and filtering and both the foliage and high contrast interfaces are very faint, which is a great start. Furthermore, Figure 39 shows imperceptible noise (foliage and interface), offering quantitative confirmation. When intersected with the variance threshold, the intermittent birds and fast-moving objects are filtered out. This is a great success. However, VAMBM still does pick up pedestrians and vehicles. It would need further supplementation or combination to reduce its false alarm rate.

\subsubsection{Demo}

This video shows the progression of the classifier. It shows the "isAll" persistence filter process and the median filtering. The ' $=$ ' characters in the filename denote a comparison of "isAll" parameters ("isAll10" and "isAll20" are shown) and median filter parameters (3x3 and $5 \times 5$ median filters are shown)

Demo: varStats_loose_isAll=_medfilt $=\_3$ fps.avi 


\subsection{Principal Component Analysis of Dual-Range Moving Average Difference (PCA-DRMAD)}

\subsubsection{Rationale}

The DRMAD shows promise as a filter that improved heat signal over background noise. Multi-temporal PCA shows movement information. PCA of the DRMAD blends the power of both by finding movement information more characteristic of heat plume. Recall that PC1 can serve as a measure of persistence, a primary attribute of heat plume, and that the deeper PC's bear information about motion, an attribute of growth.

Foliage and the pedestrian in the scene persistently raise the greatest challenge by their motion's similarity to that of the heat plume. However, PCA offers a much richer set of information about movement than VSS features. Maybe thresholds can be found within each PC image that bound heat. Furthermore, maybe they can be automatically determined, by considering that the first 1000 frames contain only foliage and a pedestrian, and very little heat, thus "setting the bar" that measures the heat plume.

\subsubsection{Experiment}

1. DRMAD was computed for the ranges shown in 
Table 21. Refer to Section 3.1.5, the experiments of which furnished the data re-used in this experiment. 
Table 21 - PCA-DRMAD Difference Ranges (Cases)

\begin{tabular}{|l|ll|}
\hline Difference Case & $\begin{array}{l}\text { Short-Range } \\
\text { EWMA p-Parameter }\end{array}$ & $\begin{array}{l}\text { Long-Range } \\
\text { EWMA p-Parameter }\end{array}$ \\
\hline 1 & 0.10 & 0.02 \\
\hline 2 & 0.07 & 0.02 \\
\hline 3 & 0.07 & 0.01 \\
\hline 4 & 0.07 & 0.008 \\
\hline 5 & 0.05 & 0.02 \\
\hline 6 & 0.05 & 0.01 \\
\hline 7 & 0.05 & 0.008 \\
\hline
\end{tabular}

2. PCA was performed, as presented in Section 3.2, with Autolinear mapping with $1 \%$ outlier rejection, calibrated at frame 3000, which has a prominent plume.

3. The maximum and minimum were found over the first 1000 frames, for each of the PC images individually.

4. The PC images were thresholded, as found in Step 3. See Figure 40.

5. Error Analysis was performed (Section 5.1.1). See Figure 41 for the categorized detection count, and Table 22 for the associated channel names and total error rate. Note that there were other minor sources of error beyond the main categories, hence the Non-Heat count in the table will not exactly equal the sum of the non-heat categories in the figure, but they will be comparable. 


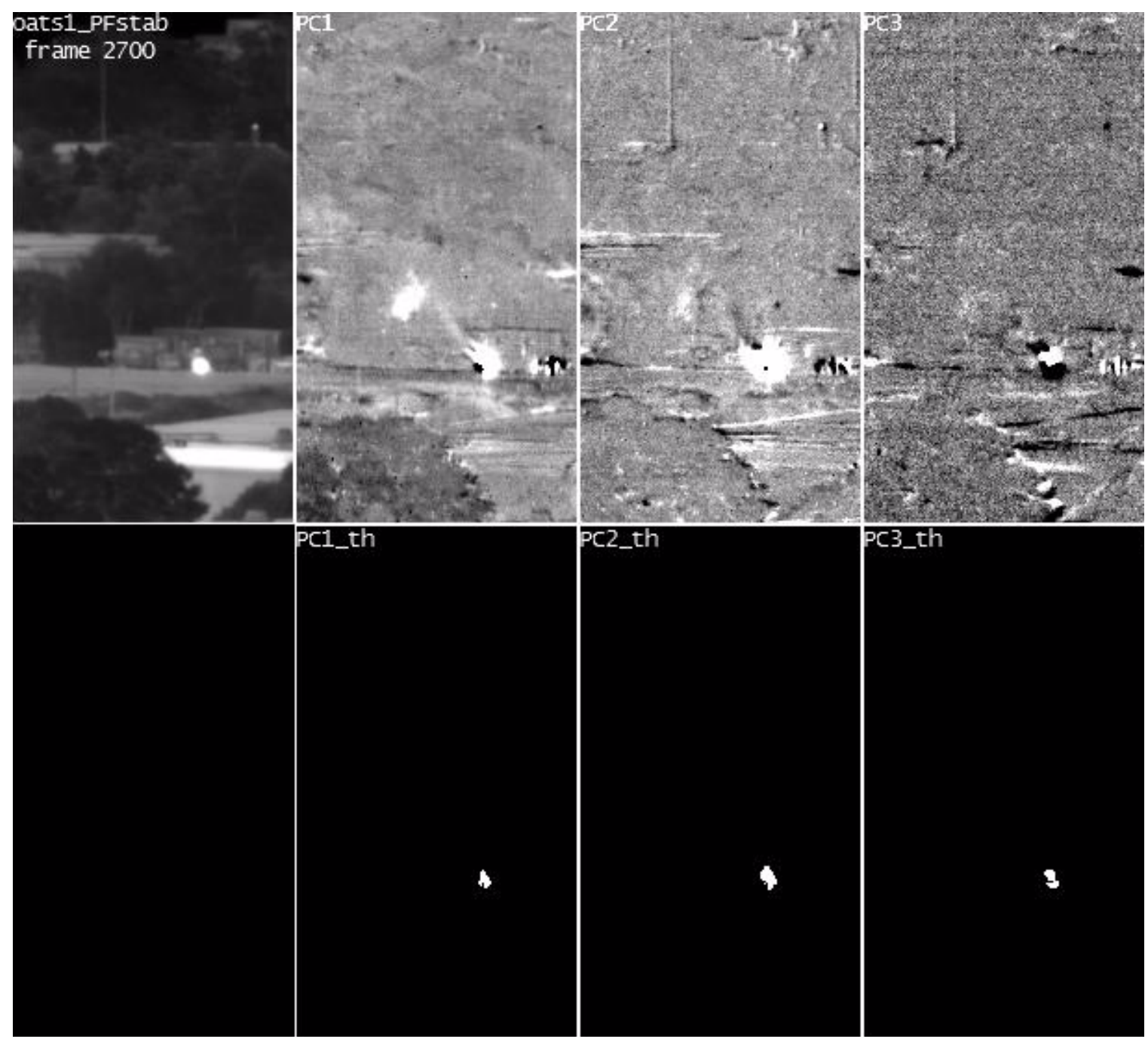

(a) 


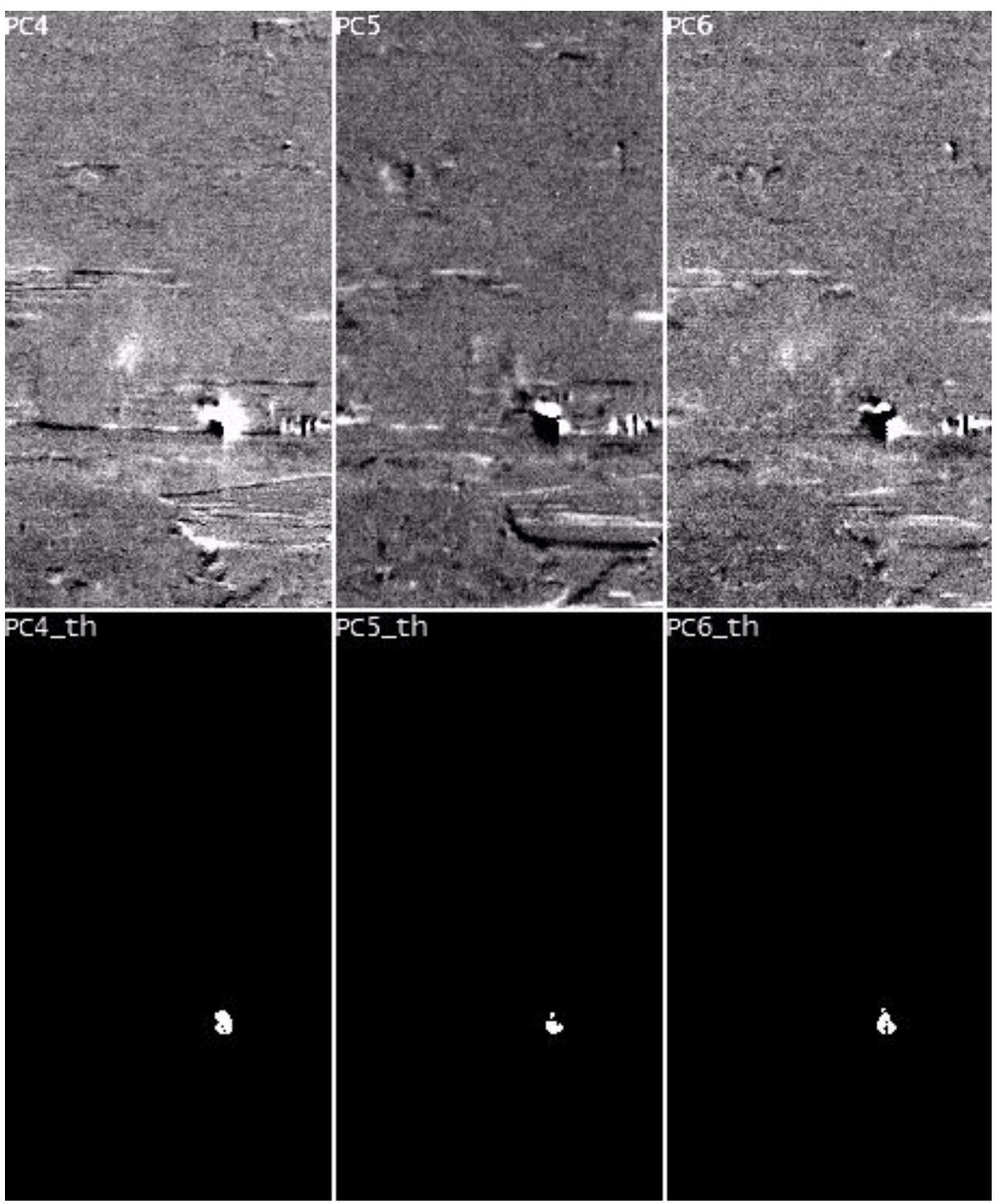

(b)

Figure 40 - PCA-DRMAD with Automatic Threshold (Demo: EWMA0.05-0.01_PCA6_auto-th.avi) 


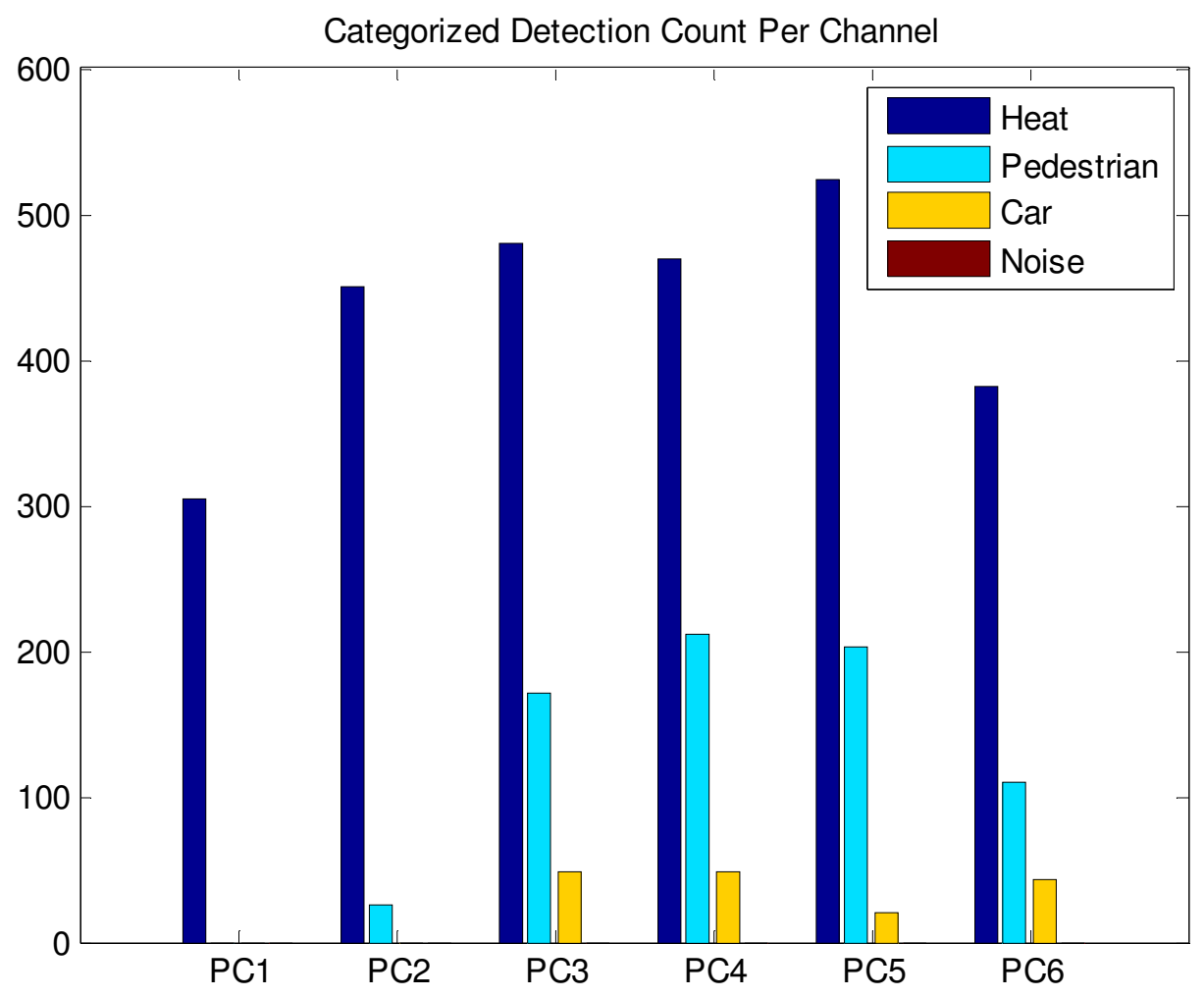

Figure 41 - CDC Comparing Auto-Thresholded PC Images

(Difference Case 1)

Table 22 - Error Analysis for PCA-DRMAD

(Difference Case 1)

$\begin{array}{llll}\text { Channel name: } & \text { Heat } & \text { Not } & \text { E_avg } \\ \text { EWMA0.07-0.02_PCA6_autolinear_auto-th } \backslash 1 & 304 & 43 & 0.026 \\ \text { EWMA0.07-0.02_PCA6_autolinear_auto-th } \backslash 2 & 450 & 116 & 0.071 \\ \text { EWMA0.07-0.02_PCA6_autolinear_auto-th } \backslash 3 & 479 & 197 & 0.070 \\ \text { EWMA0.07-0.02_PCA6_autolinear_auto-th } \backslash 4 & 469 & 231 & 0.069 \\ \text { EWMA0.07-0.02_PCA6_autolinear_auto-th } \backslash 5 & 523 & 217 & 0.099 \\ \text { EWMA0.07-0.02_PCA6_autolinear_auto-th } \backslash 6 & 382 & 144 & 0.057\end{array}$

PCA-DRMAD filters out every leading challenge category in PC1, detecting only 43 non-heat pixels throughout the entire video's test range (frames 510 to 5000), while retaining the heat plume signal. The deeper PC's also detect much heat plume, but also detect increasing proportions of non-heat, especially pedestrian.. However, inspection of Figure 40 shows that in frame 2700, the heat-related activity appears localized to the same space across all PC images. 
Viewing the video corroborates this observation. This spatial correlation can aid in the classification system.

\subsubsection{Principle Component Image Combination}

Since the auto-thresholded PC1 was able to filter out most unwanted noise sources, it will be used as a necessary condition. That is, for a pixel to count as heat plume, it must be near PC1. Since PC1 has a small signal, it will offer benefit of doubt by dilating by radius 3 , because the maximum distance from the heat plume in $\mathrm{PC} 1$ to the furthest point of heat plume in a higherorder PC was observed to be approximately 3 (future work could tune or adapt this fixed parameter). The only remaining requirement is that one other auto-thresholded PC image vote for the pixel. Put simply, a pixel will be classified as a heat plume pixel if it is a member of the dilated PC1 image and at least one other PC image.

The test is performed on all seven difference cases in 
Table 21. Finally, an error analysis is performed to compare each of the resultant classifiers, same as before. Figure 42 shows the comparison based on the difficulty categories presented in Section 5.1.1. Figure 43 shows the error rate through time index. Table 23 shows the total true positives and false positives and error rate for each channel, corresponding to those in Figure 42. Again, the names in Table 23 correspond to the data channels referred to in the code, for the reader who attempts to reproduce the work.

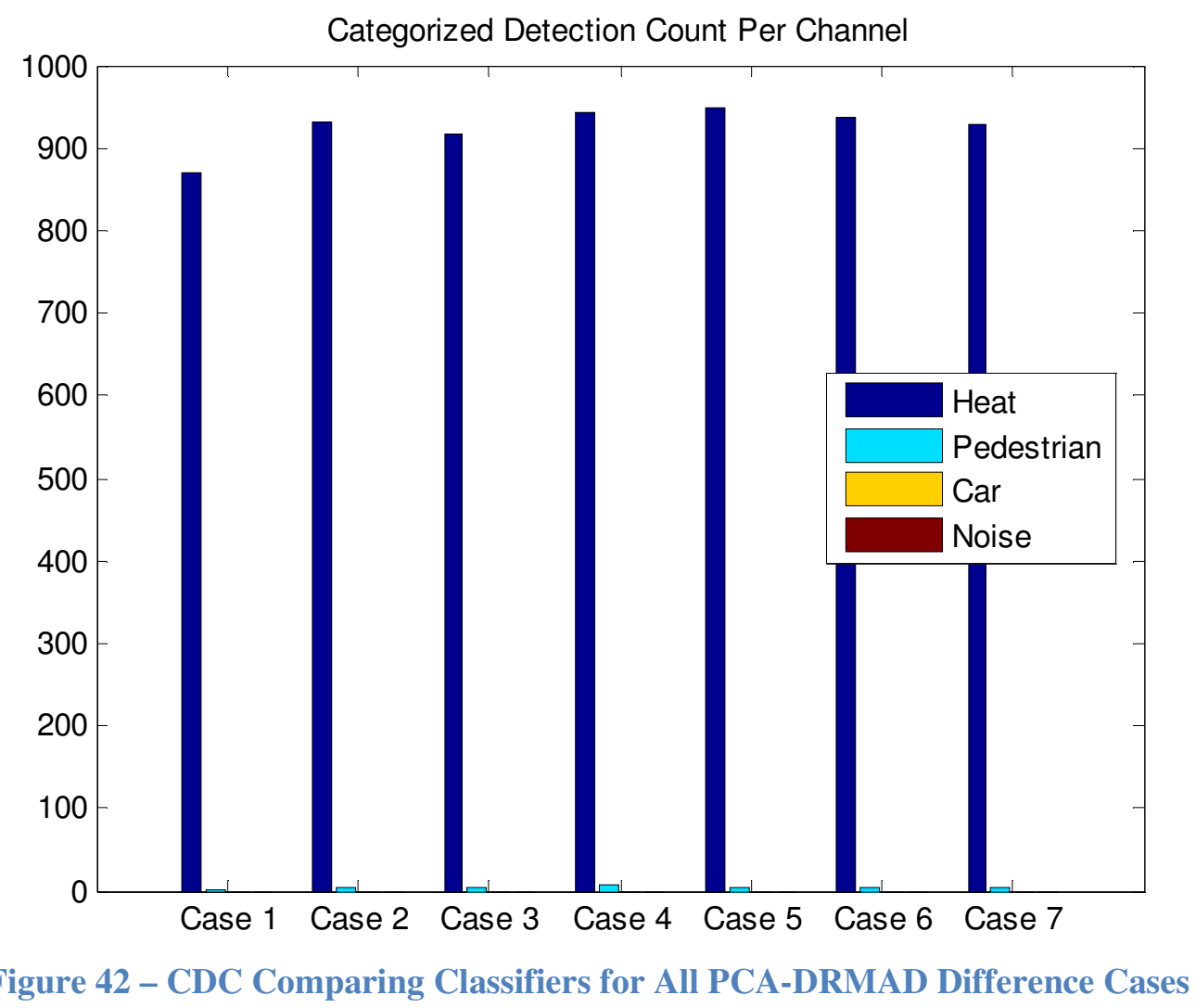



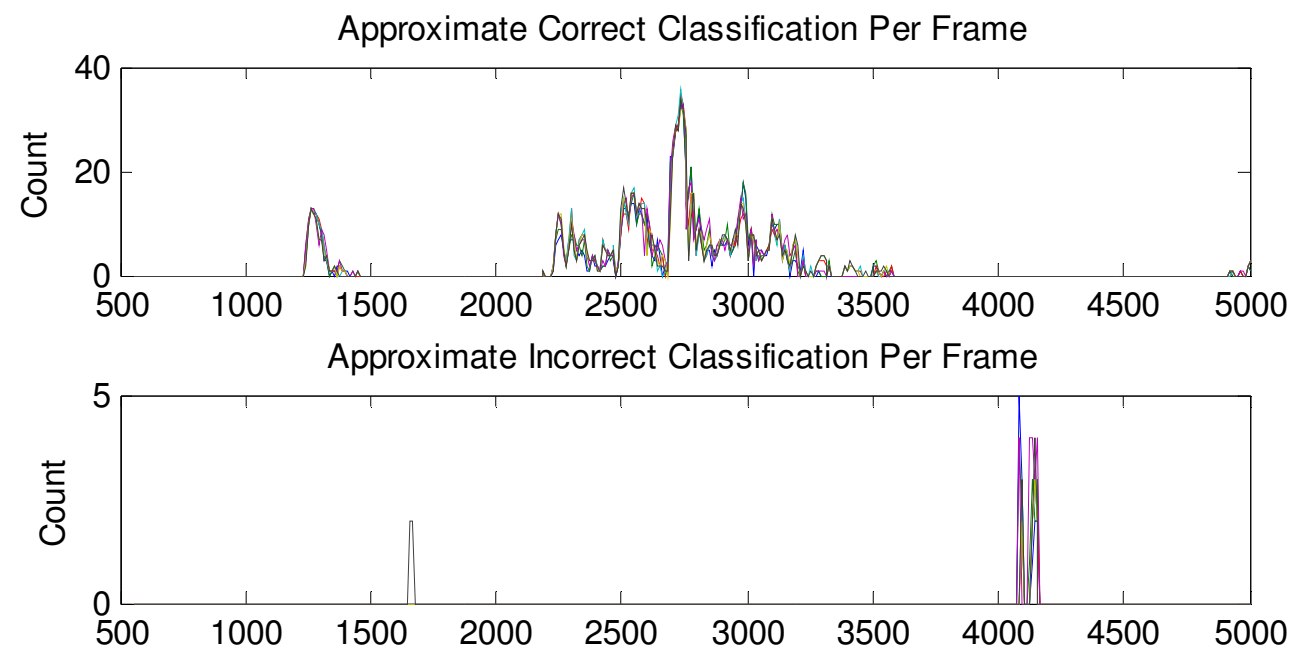

Figure 43 - Comparison of Classifiers for All PCA-DRMAD Difference Configurations

Table 23 - Error Analysis for All PCA-DRMAD Difference Configurations

$\begin{array}{lllll}\text { Case } & \text { Channel name: } & \text { Heat } & \text { Not } & \text { E_avg } \\ 1 & \text { EWMA0.1-0.02_PCA6_autolinear_auto-th_c1 } & 871 & 15 & 0.013 \\ 2 & \text { EWMA0.07-0.02_PCA6_autolinear_auto-th_c1 } & 931 & 17 & 0.016 \\ 3 & \text { EWMA0.07-0.01_PCA6_autolinear_auto-th_c1 } & 918 & 11 & 0.011 \\ 4 & \text { EWMA0.07-0.008_PCA6_autolinear_auto-th_c1 } & 944 & 10 & 0.009 \\ 5 & \text { EWMA0.05-0.02_PCA6_autolinear_auto-th_c1 } & 950 & 23 & 0.013 \\ 6 & \text { EWMA0.05-0.01_PCA6_autolinear_auto-th_c1 } & 937 & 9 & 0.009 \\ 7 & \text { EWMA0.05-0.008_PCA6_autolinear_auto-th_c1 } & 928 & 15 & 0.013\end{array}$

Inspection of Figure 42 shows that every PCA-DRMAD difference case works well, and "the best one" isn't obvious. However, looking at the average error rates in Table 23 shows a clear winner in case 6 . It has the third highest true positive rate, and the least false positive rate, which arguably carries more merit, as a decent detector may detect a fire before much time passes, but false positives make each alarm less meaningful.

Table 24 - Computation Time of PCA-DRMAD Classifier

Process Time (s)

\section{Compute DRMAD}




\begin{tabular}{|c|c|}
\hline Compute EWMA & 326 \\
\hline EWMA Subtraction & 26 \\
\hline Perform PCA & 102 \\
\hline Threshold PCA & 68 \\
\hline Total Time: & $522 \mathrm{~s}$ \\
\hline Capable Frame Rate: & 4490 frames $/ 522 \mathrm{~s}=\mathbf{8 . 6} \mathbf{f p s}$ \\
\hline
\end{tabular}

\subsubsection{Demo}

This video shows the original (stabilized) frames and the PCA-DRMAD classified frames side-by-side. Note it is case 2, which didn't have the optimal error rate, but the difference isn't discernable to the keen observer, so this suffices as a demo.

Demo: EWMA0.07-0.02_PCA6_autolinear_auto-th_c1.avi 


\subsection{Support Vector Machine (SVM)}

\subsubsection{Introduction}

A SVM trains from a set of observations belonging to a known binary class, then it classifies new observations based upon its trained state [21]. The training is done by transforming the training observations into higher-dimension space in which the two classes become linearly separable. When the separating hyper-plane transforms back into input space, it becomes a curve that bounds the classes. In this case, the SVM uses a radial basis function (RBF) kernel.

\subsubsection{Training}

SVM needs a set of truth data to train from. That is, it needs a training set representative of heat plume movement, which means the heat plume's location must be exactly known to train it. The challenge is that the heat plume constantly moves. To solve this problem, a mask can be drawn around the approximate are of the heat plume, including all approximate area where heat touches, and then set a threshold on the variance video, and apply a binary intersection. After these operations are done, the output video sequence looks like the clip shown in Figure 44.

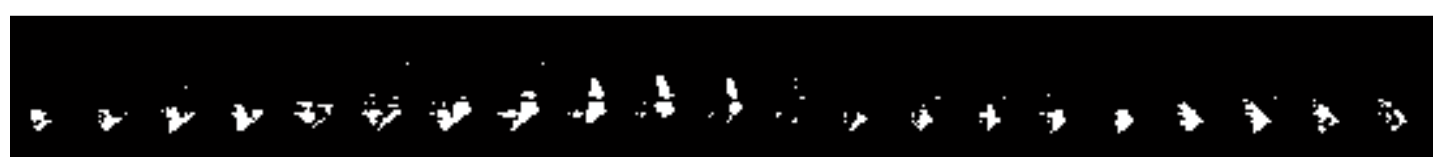

Figure 44 - Train_var30 Mask, “Heat Mask” (frames 3030 - 3230, step 10)

The SVM also needs negative training data. For this purpose, a mask was created that selects everything but the fire and heat area. Pixels were selected at random from this mask to collect signal sequences from as negative training data, as the non-heat mask area far exceeds the heat region mask in area. Every pixel within the heat mask was used to collect underlying sequences for positive training data. These methods resulted in an approximately proportionate sizes between positive and negative training sets, totaling about 44,000 training samples.

There were two sets of signals used for SVM training data. 
1) One set was the PCA set (Section 3.2); that is, a single training sample was a 6dimensional vector taken from one pixel of the PCA set, where the dimensions corresponded to the PC images. The PC images were unmapped.

2) The other was also from the PC images, but in an effort to incorporate more timerelated information as well as more meaningful information, the training data consisted of the first $3 \mathrm{PC}$ images at time $t_{f}$, in conjunction with the first $3 \mathrm{PC}$ images at time $t_{f}-10$ (that is, the previous time index considered, as the output time resolution is divided by 10 , to be $3 \mathrm{fps}$.

When training off the first data set, a 5-fold stratified cross-validation method was performed, wherein the data set is divided into 5 sets, and 4 are used for training while the remaining set is used for validation. The process is repeated for a different set of 4 and 1 until all combinations have been tried. This process trained the SVM to have 5.4\% error rate.

\subsubsection{Results}

Unfortunately, the SVM was unable to learn the general distinction between heat and non-heat, for both sets of training data. For approximately 44,000 training vectors, there were approximately 11,000 support vectors. Figure 45 shows the results. Both trainings appear homogeneously incorrect. This failure may be due to any of the following reasons:

- $\quad$ The training mask may be faulty.

- The training mask and the matching heat signature may be asynchronous.

- The training mask is defined for individual discrete moments of time to a resolution of 0.33 seconds, yet is used to identify and collect information over 2 seconds. The disparity may be too great. 


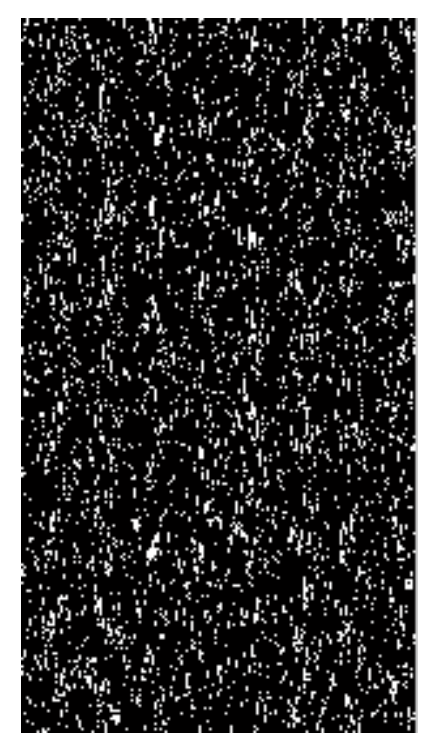

(a) PCA6_nomap

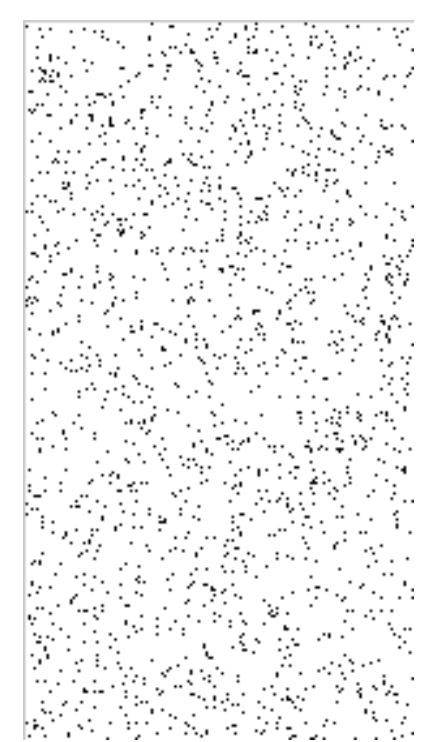

(b) PCA6_delay

Figure 45 - Support Vector Machine Output 


\subsection{Classification Function Summary}

\begin{tabular}{|c|c|c|}
\hline \multirow[t]{2}{*}{ Function / Script Name } & \multicolumn{2}{|c|}{ Description Overview } \\
\hline & Function Input & Function Output \\
\hline $\begin{array}{l}\text { Classification } \\
\text { _BayesVar }\end{array}$ & \multicolumn{2}{|c|}{$\begin{array}{l}\text { Imports saved histogram data from TimeDomainAnalysis } \\
\text { as a-priori knowledge to classify variance features. }\end{array}$} \\
\hline Process_Varstats & \multicolumn{2}{|c|}{$\begin{array}{l}\text { Directs the entire process of producing, analyzing and } \\
\text { displaying MMT from feature extraction to processing to } \\
\text { classification to post-processing. Calls } \\
\text { FeatureExtraction_VarStats and Classification_VarStats. }\end{array}$} \\
\hline Process_NoiseResist & \multicolumn{2}{|c|}{$\begin{array}{l}\text { Used to investigate methods to isolate heat plume } \\
\text { movement pattern. Ultimately it discovers VAMBM, which } \\
\text { it fully implements, analyzes, and displays. }\end{array}$} \\
\hline Process_DRMAD_PCA & \multicolumn{2}{|c|}{$\begin{array}{l}\text { Directs the process of producing, analyzing, and displaying } \\
\text { PCA-DRMAD, assuming the EWMA features have already } \\
\text { been extracted (FeatureExtraction_EWMA). Calls } \\
\text { Process_EMAsub, FeatureProcessing_PCA, ErrorAnalysis. }\end{array}$} \\
\hline Process_EMAsub & \multicolumn{2}{|c|}{$\begin{array}{l}\text { Computes several EWMA DRMADs, permuting several } \\
\text { EWMA parameters: e.g. } 0.1,0.07,0.05,0.02,0.01,0.008 \text {. } \\
\text { This data supplies Process_NoiseResist. }\end{array}$} \\
\hline $\begin{array}{l}\text { Classification } \\
\text { _VarStats }\end{array}$ & $\begin{array}{l}\text { Source and output path, } \\
\text { frame interval. Output } \\
\text { AVI path. }\end{array}$ & $\begin{array}{l}\text { Implements thresholding and } \\
\text { intersection of varMean and } \\
\text { varMax, to produce "loose", } \\
\text { "tight", and "config3". Creates } \\
\text { video of results. }\end{array}$ \\
\hline $\begin{array}{l}\text { Classification } \\
\text {-Training }\end{array}$ & \multicolumn{2}{|c|}{$\begin{array}{l}\text { Script that creates the training mask (Figure } 44 \text { ) from a } \\
\text { manual mask intersected with thresholded variance. }\end{array}$} \\
\hline Classification_SVM & $\begin{array}{l}\text { Source channel, path of } \\
\text { training data (produced } \\
\text { from } \\
\text { ExtractTrainingData } \\
\text { _PCA) }\end{array}$ & $\begin{array}{l}\text { Confusion matrix of the support } \\
\text { vector machine's classified } \\
\text { output. }\end{array}$ \\
\hline $\begin{array}{l}\text { ExtractTrainingData } \\
\text { _PCA }\end{array}$ & $\begin{array}{l}\text { Source channel, moving } \\
\text { mask path (modeled as a } \\
\text { classification), }\end{array}$ & $\begin{array}{l}\text { Sets of affirmative and negative } \\
\text { training data, collected from } \\
\text { source channel beneath the } \\
\text { moving mask. }\end{array}$ \\
\hline ErrorAnalysis & $\begin{array}{l}\text { A set of classifications } \\
\text { under test, frame range. }\end{array}$ & $\begin{array}{l}\text { Outputs error rates for each } \\
\text { classification, and plots bar } \\
\text { graph of what they truly } \\
\text { classified by category. }\end{array}$ \\
\hline
\end{tabular}




\section{CONCLUSION}

This thesis has covered the search for and study of features (frames thereof) that represent information about heat plume's characteristic behaviors of persistence and growth. It has discussed how these features were combined to harness the unique information that each feature offered towards emphasizing the distinction between heat plume and not. Classifiers were designed and tested to justify their final parameters, and to compare the relative quality of the methods, side-by-side.

\subsection{Feature Conclusions}

The features were chosen because they all represented different aspects of time-domain information. While tuning the more successful features in pursuit of error minimization, some lessons emerge. The VSS classifiers suffered less error when the set size was reduced from 20 to 15 to 10 , demonstrating that heat plume information demands finer measurement. A shortcoming of the VSS methods is that a small set size compromises the statistics calculated over the set, making it a tool of a broader scope, perhaps less suited to this application than PCA-DRMAD. By its nature, a dual-range moving average difference allows for finer control than VSS because the parameter of the filter can freely vary, where the VSS parameters suffer more dramatically if they aren't within a conducive range. Hence DRMAD more easily enhanced the signal. Furthermore, DRMAD optimized the error rate when its p-parameters were $p_{\text {long }}=0.05$ and $p_{\text {short }}=0.01$, which corresponds to a particular frequency range, implying that frequency is relevant, which VSS cannot directly interact with: variance is not sensitive to order or frequency content. By the same token, PCA also does not consider frequency content directly, rather it considers varying degrees of how much change occurs within its time samples. This is still an order of magnitude finer than VSS, and precise frequencies aren't important when the heat plume itself exhibits much chaotic behavior. 


\subsection{Comparison of Detection Methods}

Table 25 gives an overview of the sensitivity of each classifier to each leading challenge category. Every error rating should be regarded as a preliminary figure, with the potential to decrease with judicious further filtering and combining.

Table 25 - Classifier Sensitivity - Categorized Detection Count

\begin{tabular}{llllll}
\hline & Heat & Noise* & Cars & Pedestrian & Avg Error \\
\hline Ideal & $>0$ & 0 & 0 & 0 & 0 \\
Variance Corridor & N/A & N/A & N/A & N/A & N/A \\
MMT & 1700 & 0 & 6600 & 722 & $48.2 \%$ \\
VAMBM & 2530 & 0 & 12800 & 5700 & $62.4 \%$ \\
PCA-DRMAD & 937 & 0 & 0 & 5 & $0.9 \%$ \\
SVM & N/A & N/A & N/A & N/A & N/A \\
\hline
\end{tabular}

*Noise refers to interface noise

Table 26 - Comparison of Classifier Computation Time

\begin{tabular}{lll}
\hline Method & Computation Time & Frame Rate* \\
\hline Variance Corridor & N/A & N/A \\
MMT & $640 \mathrm{~s}$ & $6.0 \mathrm{fps}$ \\
VAMBM & $779 \mathrm{~s}$ & $5.7 \mathrm{fps}$ \\
PCA-DRMAD & $522 \mathrm{~s}$ & $8.6 \mathrm{fps}$ \\
\hline
\end{tabular}

*Frame rate is a superficial feature based on the prototype implementation. The principal source of delay is USB 3.0 HDD access time.

In as much as "we are what we eat," a classifier's output needs quality features to process. It follows from the Feature Conclusions that PCA-DRMAD enjoys the most advantage from a resolution and detail standpoint. Its average error rate (Precision) of $0.9 \%$ certainly heralds its own success and merit. 


\subsection{Future Work}

- Reduce false positives in VSS feature classifiers, both MMT and VAMBM, by filtering out low-persistence behavior (cars).

- Increase the sensitivity of PCA-DRMAD Classifier without reducing the error rate.

- When calibrating the automatic threshold, mask out the known fire region, to eliminate any unwarranted bias.

- Variance Set Statistics do not include any frequency information. Temporal frequency domain features may lead to more information than variance alone can offer.

Investigate common DSP notch filters such as Wiener, keeping in mind that DRMAD worked best within the frequency range corresponding to $p_{\text {long }}=0.05$ and $p_{\text {short }}=0.01$.

- Diagnose why SVM on PCA of DRMAD data failed, and fix it.

- Vary the PCA parameters on DRMAD:

- Principal component count

○ Period between time samples

- $\quad$ Try PCA on VSS features.

\subsection{Lessons Learned}

- Plan ahead for data collection missions. Consider the needs of those using the data, survey the available tools, and make use of them.

- Spend time at the beginning of a coding project to develop the tools so that every stage of the prototyping process isn't belabored. 


\section{BIBLIOGRAPHY}

[1] National Interagency Fire Center. <www.nifc.gov>. May 2012.

[2] Tyco Fire and Security.

<http://www.tycofireandsecurity.com/Capabilities/firedetection> May 22, 2012.

[3] Beam Detection, Westminster International, Ltd. < http://www.wi-

Itd.com/fire/Fire_Detection_Systems/Beam_Detectors> May 22, 2012.

[4] Wang J, Song W, Wang W, Zhang Y. "A new algorithm for Forest fire smoke detection based on MODIS data in Heilongjiang province”, Hefei University of Technology, 2011.

[5] Zhang D, Han S, Zhao J, Zhang Z, Qu C, Ke Y, Chen X. "Image Based Forest Fire Detection Using Dynamic Characteristics With Artificial Neural Networks." International Joint Conference on Artificial Intelligence, 2009.

[6] Ho C, Kuo T. "Real-Time Video-Based Fire Smoke Detection System." IEEE/ASME International Conference on Advanced Intelligent Mechatronics, 2009.

[7] Mathworks, Computer Vision System Toolbox. <http://www.mathworks.com/products/computer-vision/> May 22, 2012

[8] Lee K Y, Chuang Y Y, Chen B Y, Ouhyoung M. "Video Stabilization using Robust Feature Trajectories." National Taiwan University, 2009.

[9] Tordoff B, Murray D W. "Guided sampling and consensus for motion estimation." European Conference on Computer Vision, 2002.

[10] Rosten E, Drummond T. "Machine learning for high-speed corner detection." European Conference on Computer Vision, May 2006.

[11] Forsyth D A, Ponce J. Computer Vision, a modern approach. Prentice Hall, 2003.

[12] FLIR, "Seeing through fog and rain with a thermal imaging camera." $<$ http://www.metrum.co.uk/images/AS_FOG.pdf>, 2012.

[13] Bosch, Gomez, Vergara, Moragues. "Infrared image processing and its application to forest fire surveillance." IEEE Conference on Advanced Video and Signal Based Surveillance, pp. 283. (2007).

[14] Vergara L, Bernabeu P. Automatic Signal Detection applied to fire control by infrared digital signal processing. Signal Processing. Volume 80, pp. 659-669, 2000.

[15] Gonzalez R C, Woods R E. Digital Image Processing, $3^{\text {rd }}$ Ed. Prentice Hall, 2008.

[16] Fukunaga, Keinosuke. Introduction to Statistical Pattern Recognition. Elsevier, 1990.

[17] Shlens J. A Tutorial on Principal Component Analysis. 
[18] PHYS.ORG, New forest fire detection system prototype installed at Lake Tahoe. <http://phys.org/news179400560.html>, Dec 7, 2009.

[19] Li Z, Kaufman Y, Ichoku C, Fraser R, Trishchenko A, Giglio L, Jin J, Yu X, "A Review of AVHRR-based Active Forest Fire Detection Algorithms: Principles, Limitations, and Recommendations.

[20] Kaufman Y J, Kleidman R G, King M D. "SCAR-B Fires in the Tropics: properties and their remote sensing from EOS-MODIS.” J. Geophys. Res., 103:31,955-31,969, 1998.

[21] Haykin S. "Neural Networks and Learning Machines, 3ed.” Prentice Hall, 2009. 


\section{GLOSSARY}

\begin{tabular}{ll}
\hline Term / Acronym & Description \\
\hline CLWIR & Cooled Long-Wave Infrared. \\
\hline CMWIR & Cooled Mid-Wave Infrared. \\
\hline DRMAD & Dual-Range Moving Average Difference \\
\hline EMA/EWMA & Exponential / Exponentially-Weighted Moving Average \\
\hline PCA & Principal Component Analysis \\
\hline PF & Point Feature \\
RANSAC & Random Sample Consensus \\
SMA & Simple Moving Average \\
SVM & Support Vector Machine \\
\hline UCLWIR & Uncooled Long-Wave Infrared. \\
VAMBM & Variance-Augmented Mean-by-Median Quotient \\
\hline VSL & Variance (Set) Statistics, Loose threshold and intersection configuration \\
\hline VSS & Variance Set Statistics \\
\hline
\end{tabular}




\section{APPENDIX I - CLASSIFICATION CODE}

These functions are primarily responsible for all the algorithms and the output seen in this thesis. The entirety of the code is not included in this appendix, in the interest of space (clutter), however the full code should accompany this document as an attachment. The following functions are included herein. The lower levels in the list indicate that the function is called by the higher level function or script.

- Classification_BayesVar

- Process_VarStats.m

- FeatureExtraction_VarStats

- Classification_VarStats

- Process_NoiseResist.m

- FeatureExtraction_VarStats

○ FeatureProcessing_Simple

- Process_DRMAD_PCA.m

- Process_EMAsub.m

- Classification_SVM

○ ExtractTrainingData_PCA.m 


\section{Classification_BayesVar.m}

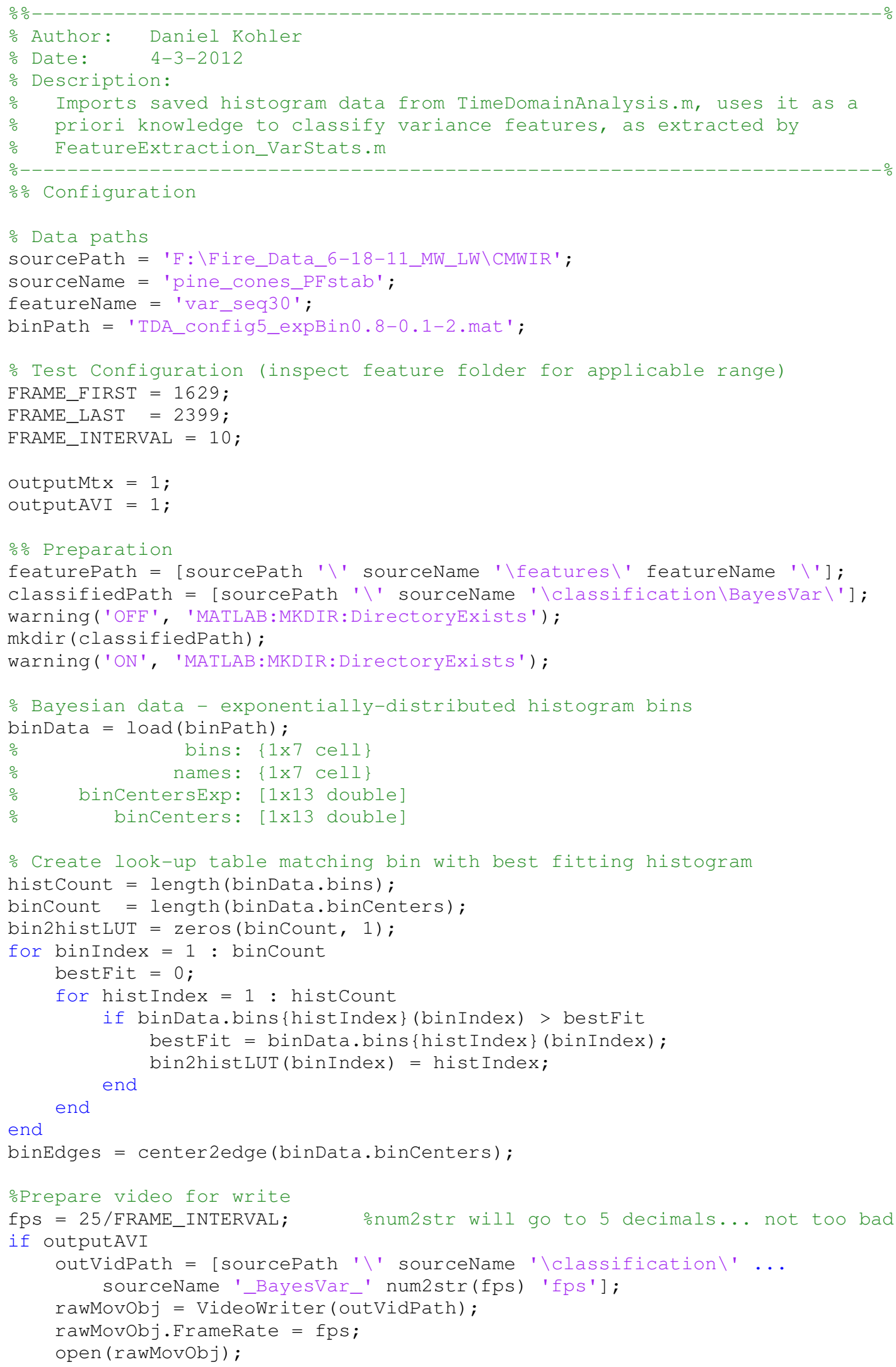




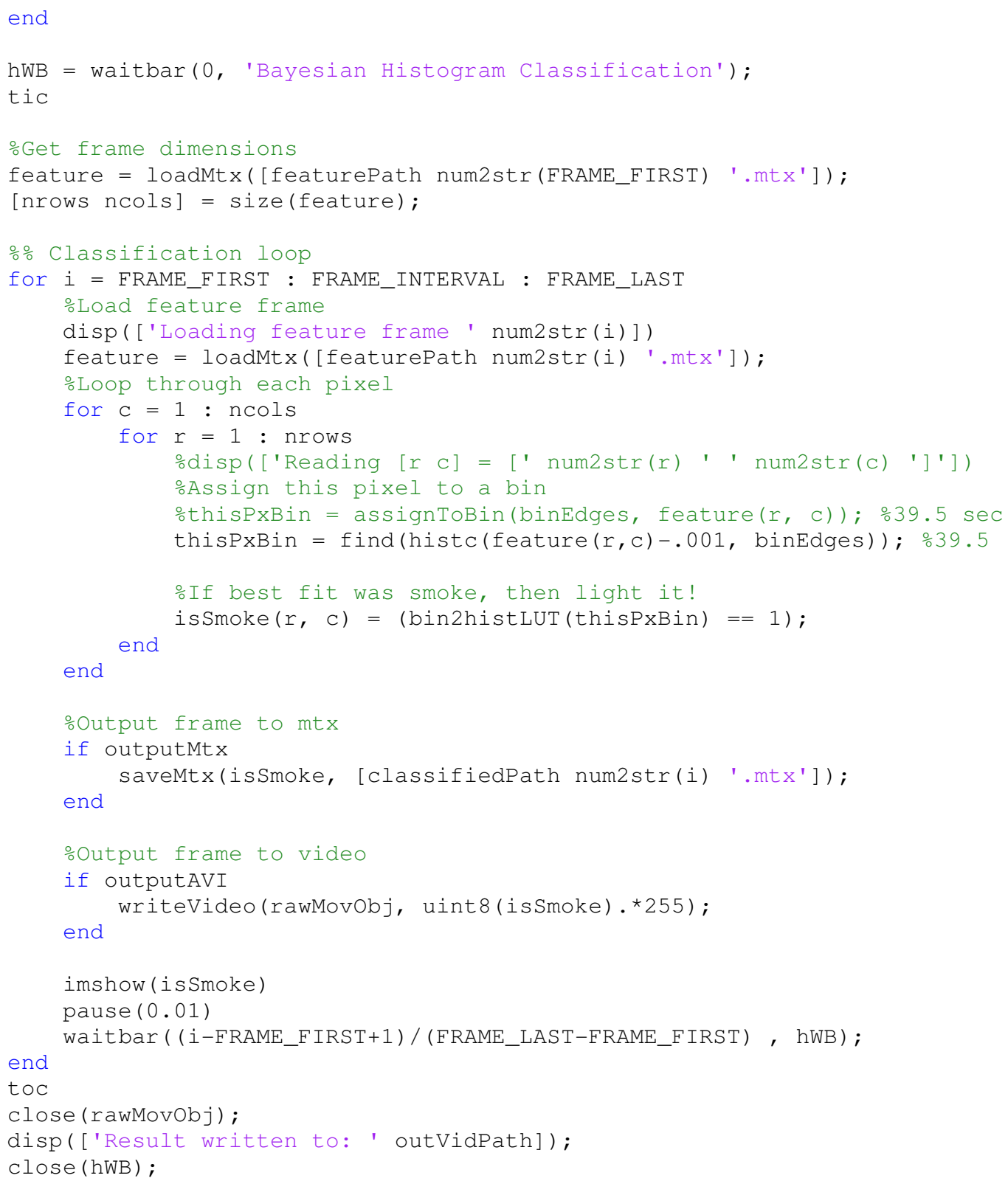




\section{Process_VarStats.m}

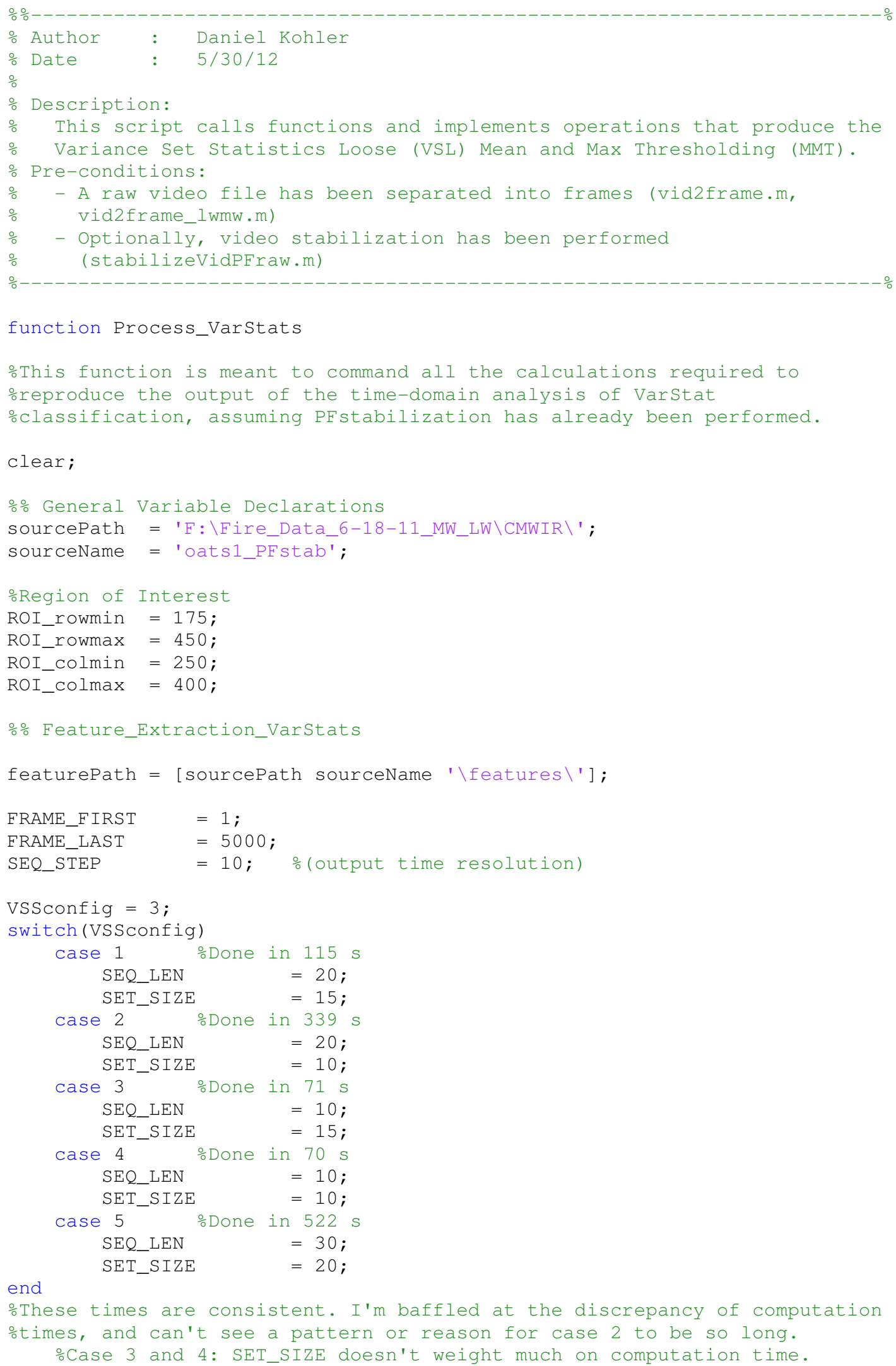




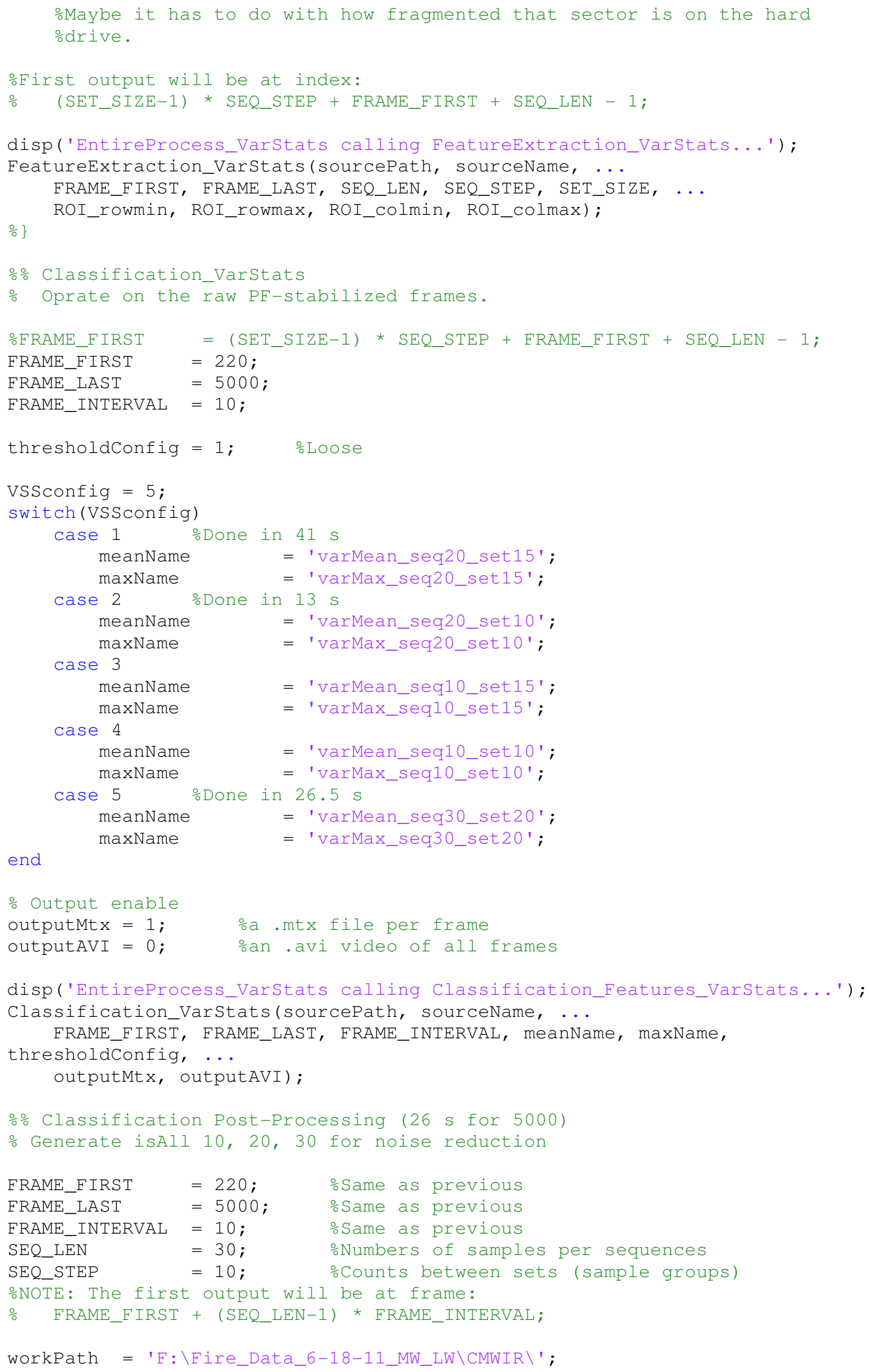




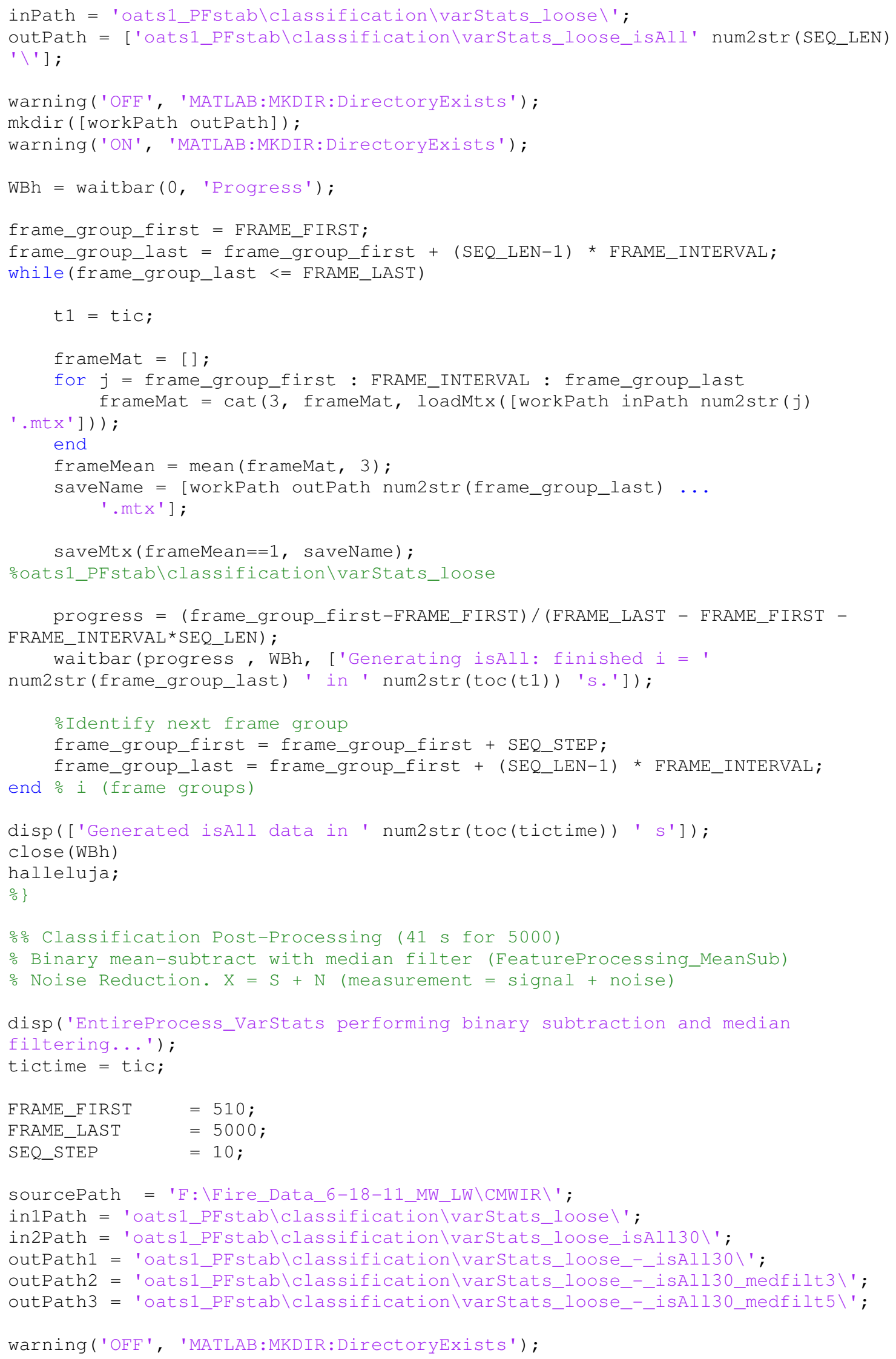




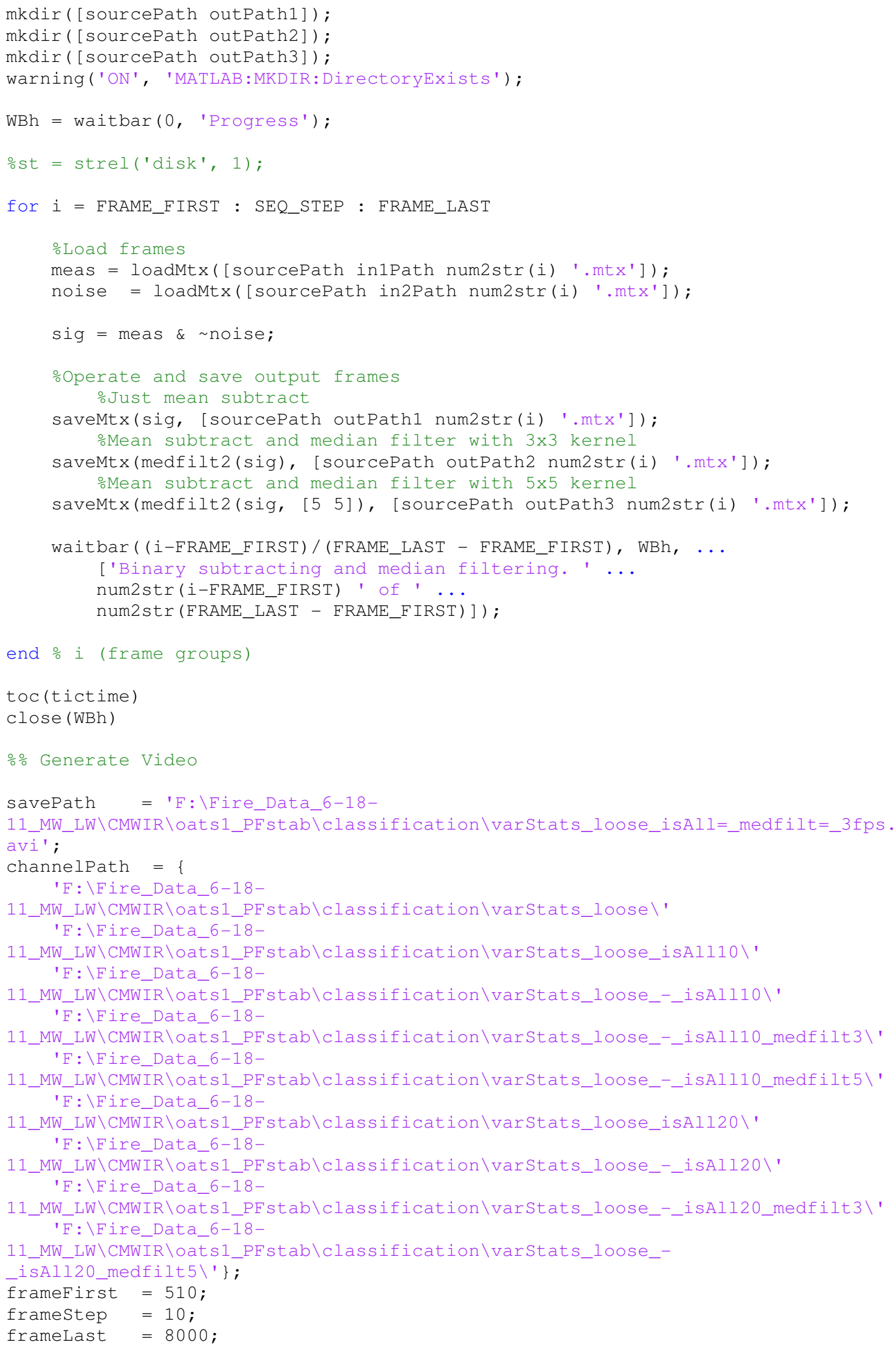




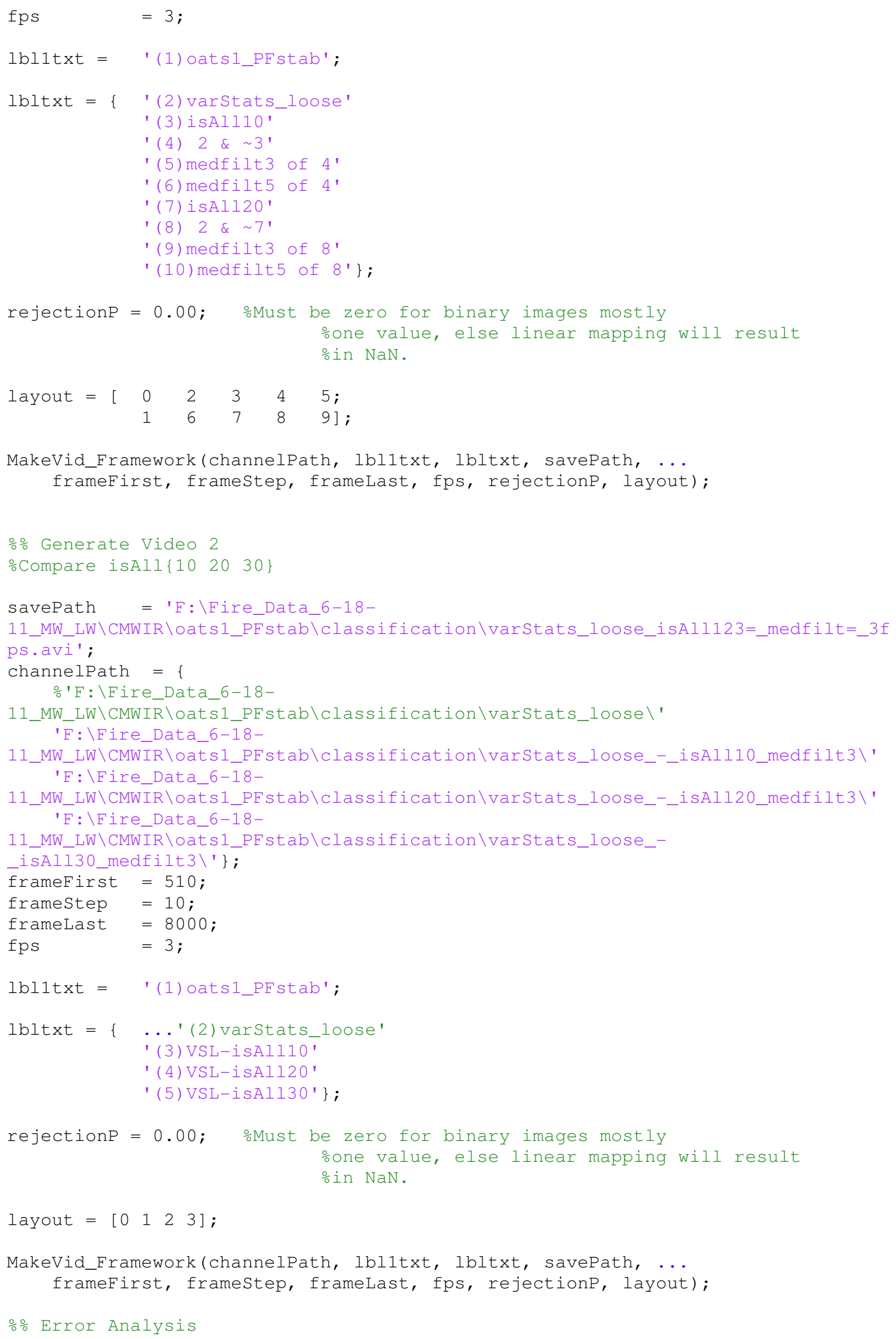




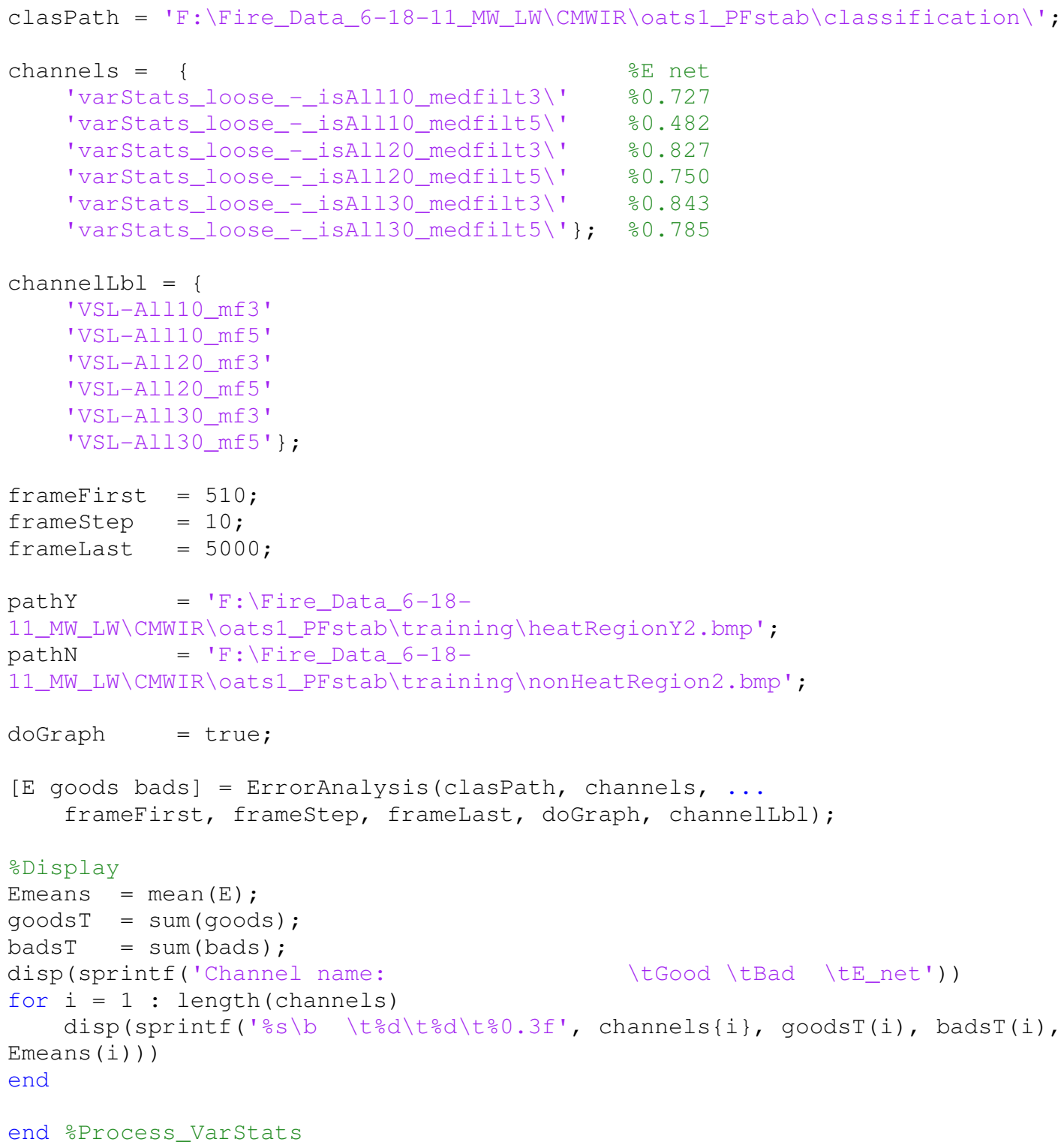




\section{FeatureExtraction_VarStats.m}

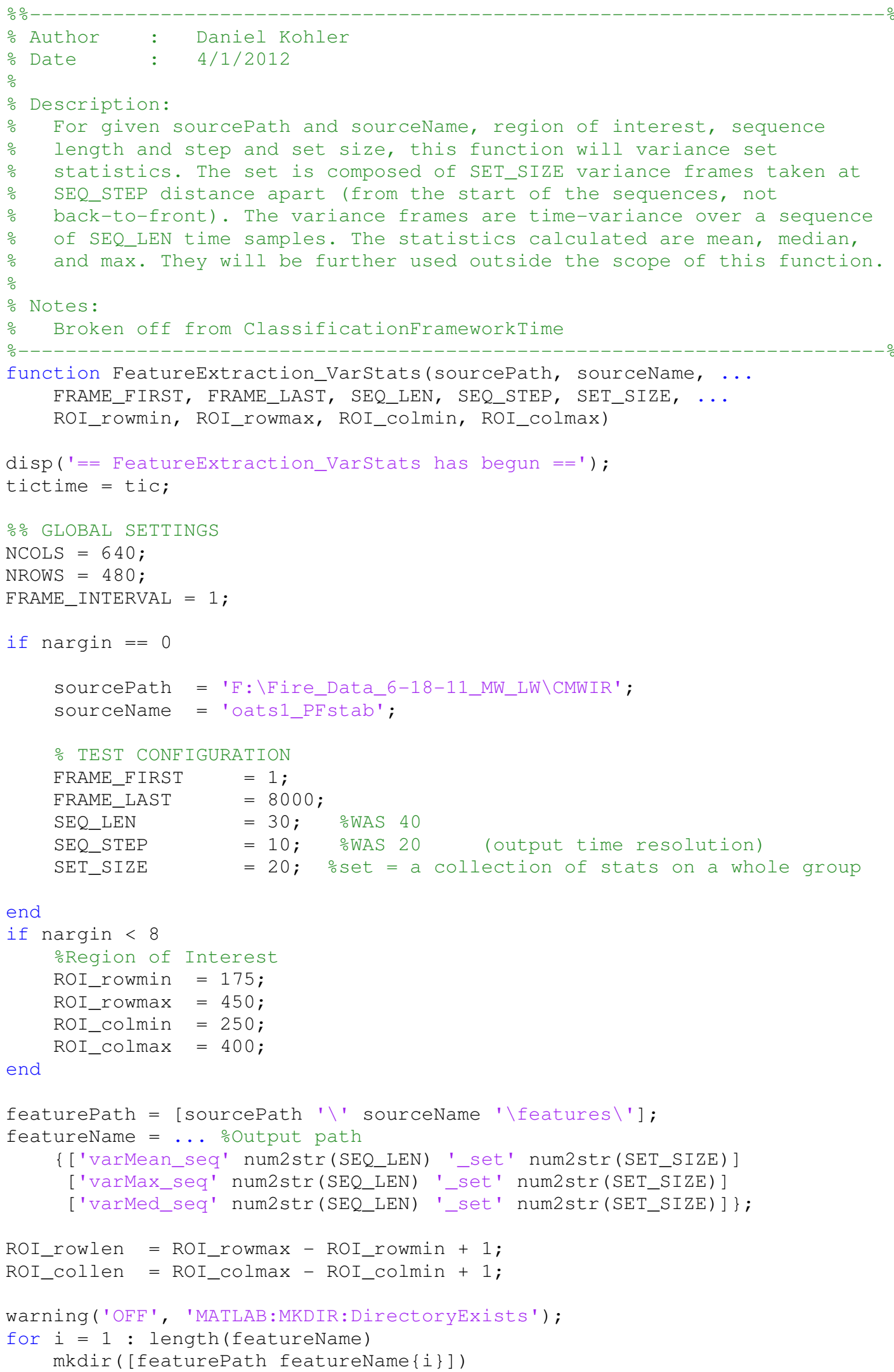




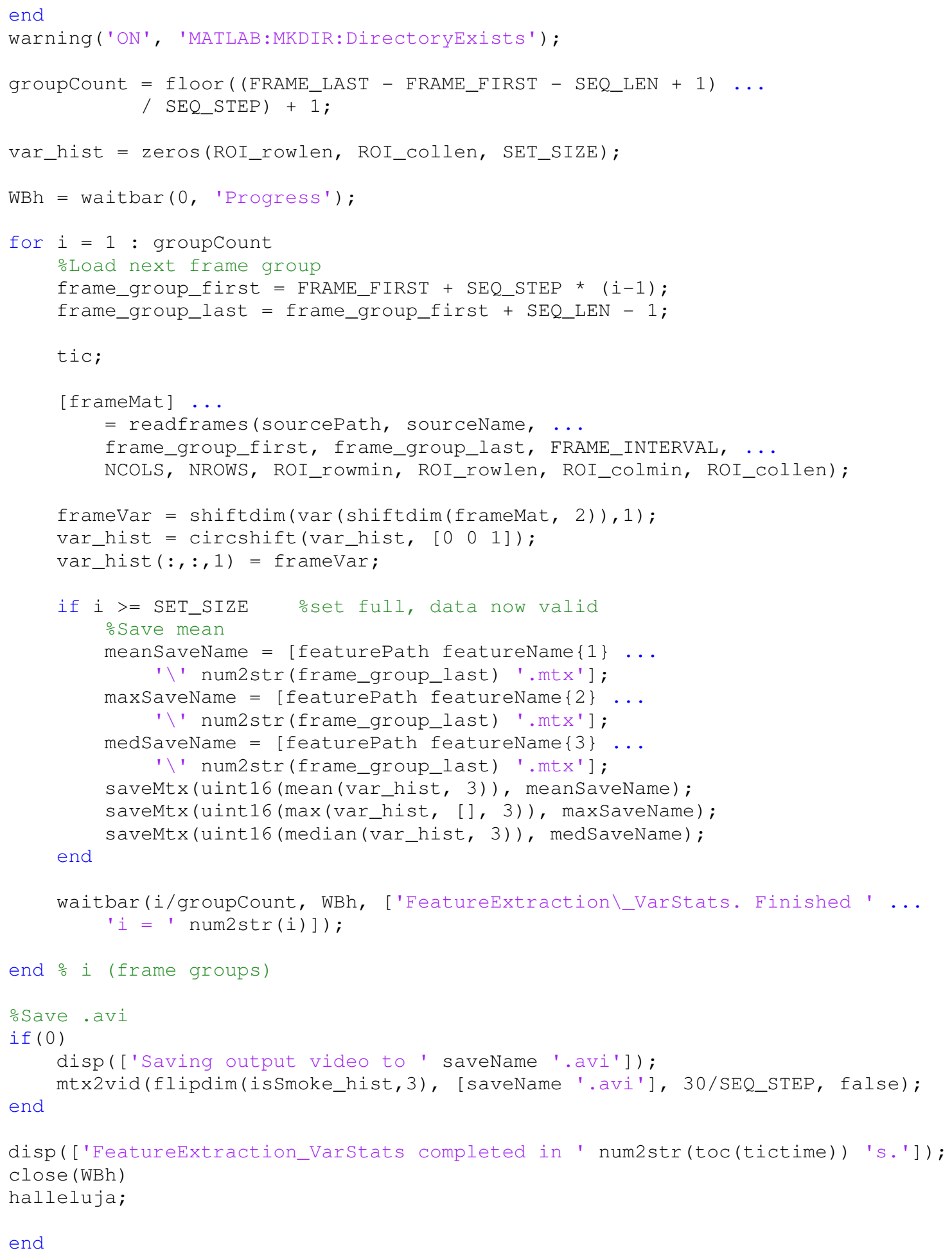




\section{Classification_VarStats.m}

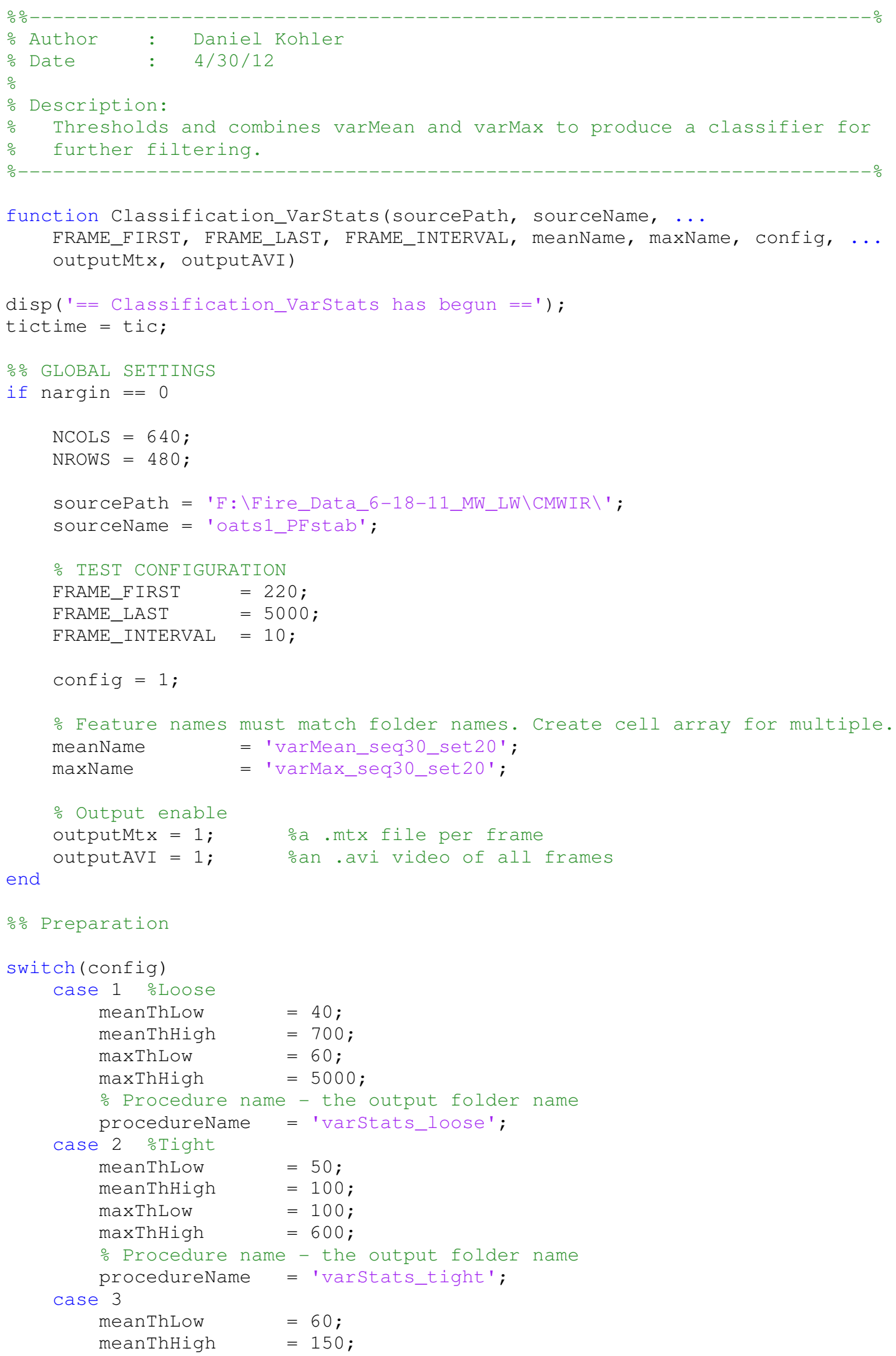




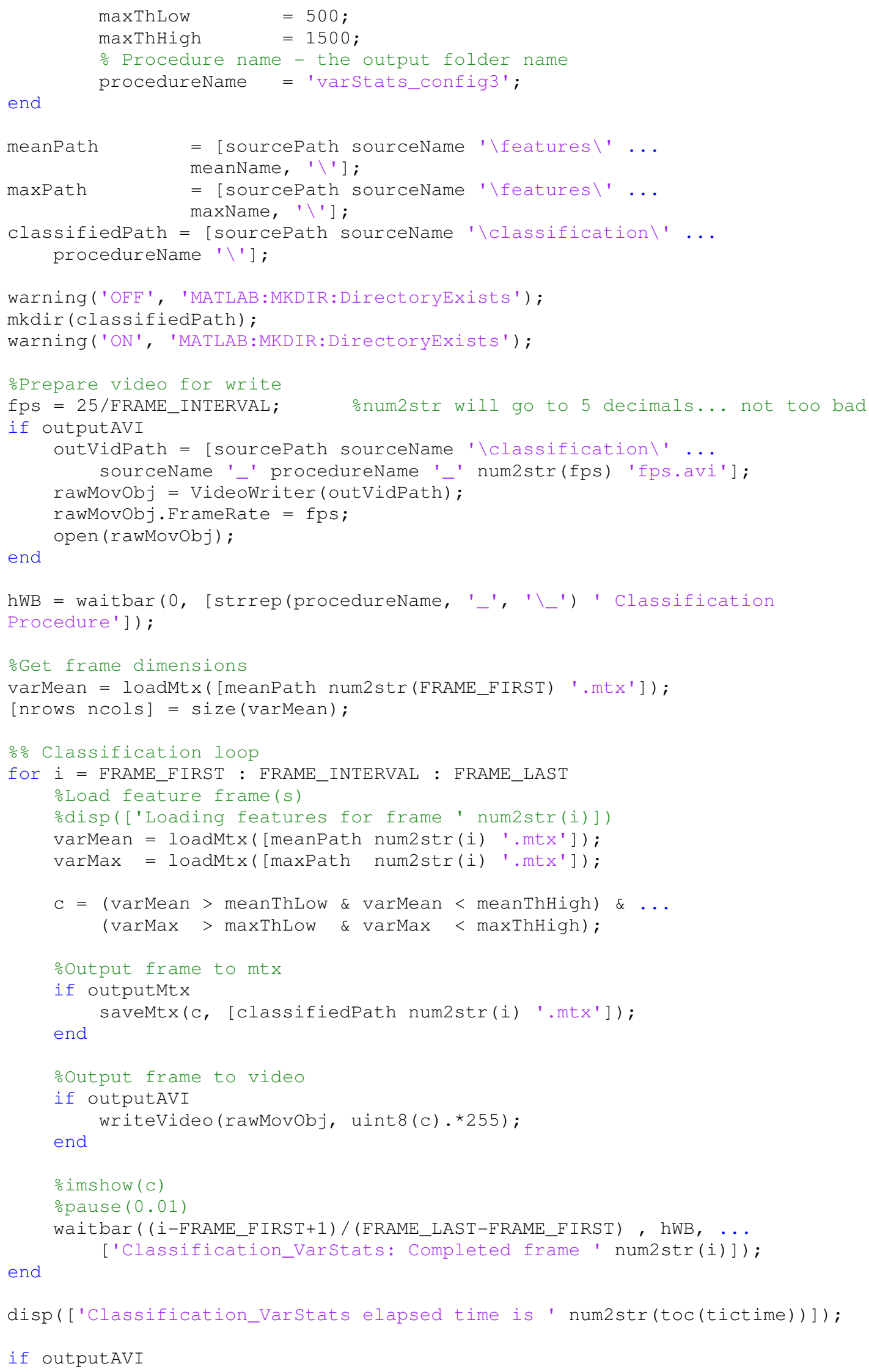


close (rawMovObj);

end

disp(['Result written to: ' outVidPath]);

halleluja;

close (hWB);

clear;

end 


\section{Process_NoiseResist.m}

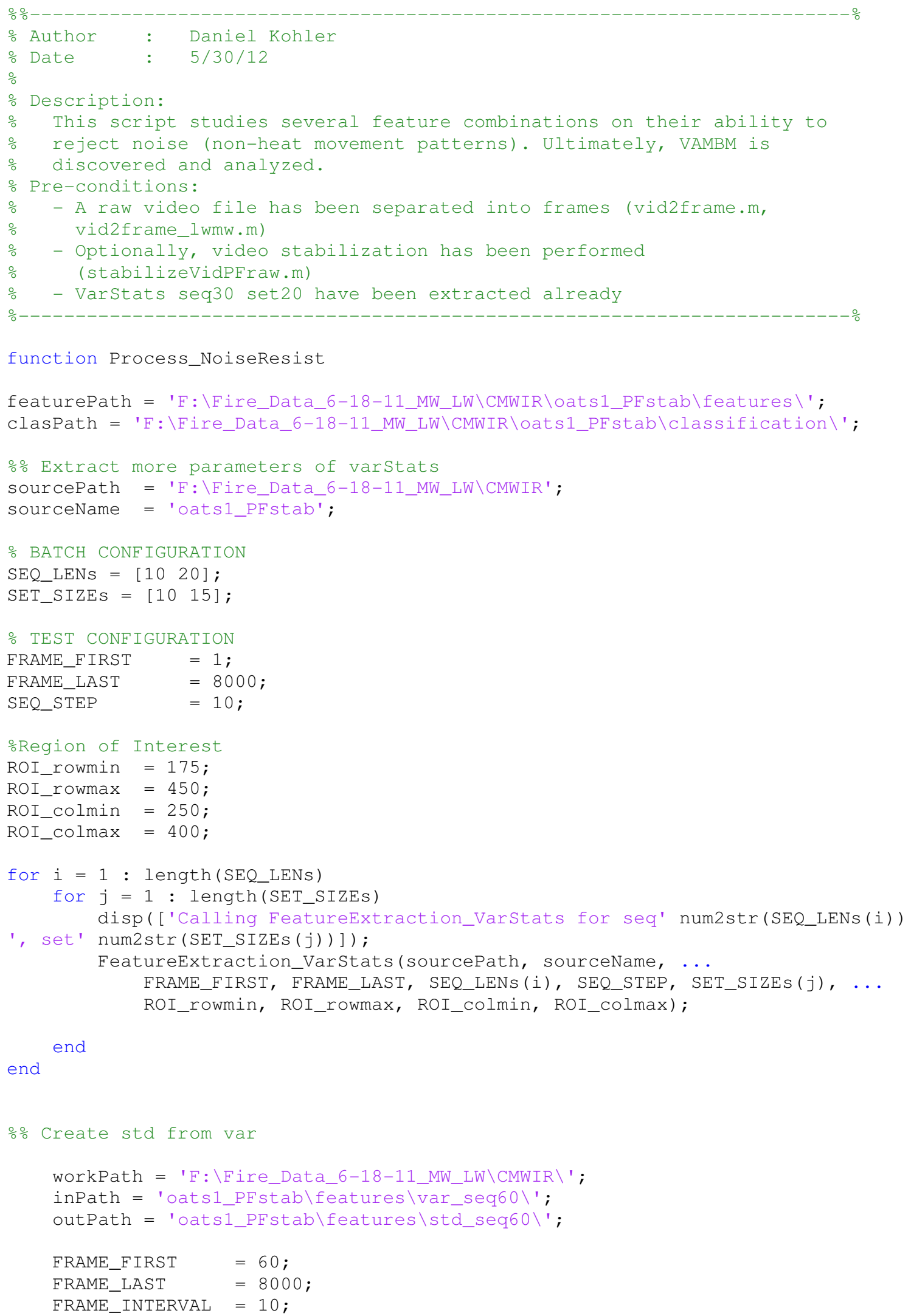




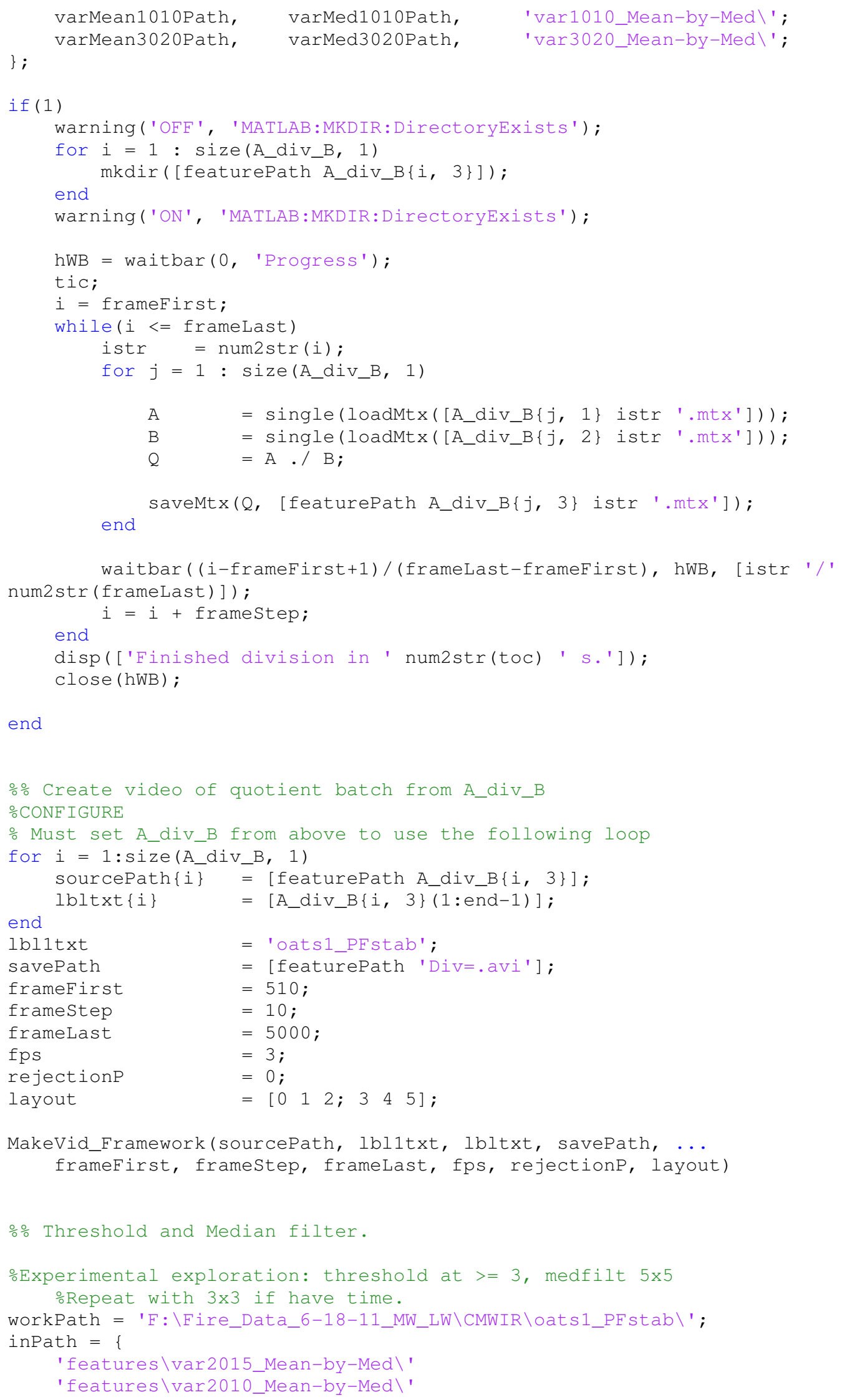




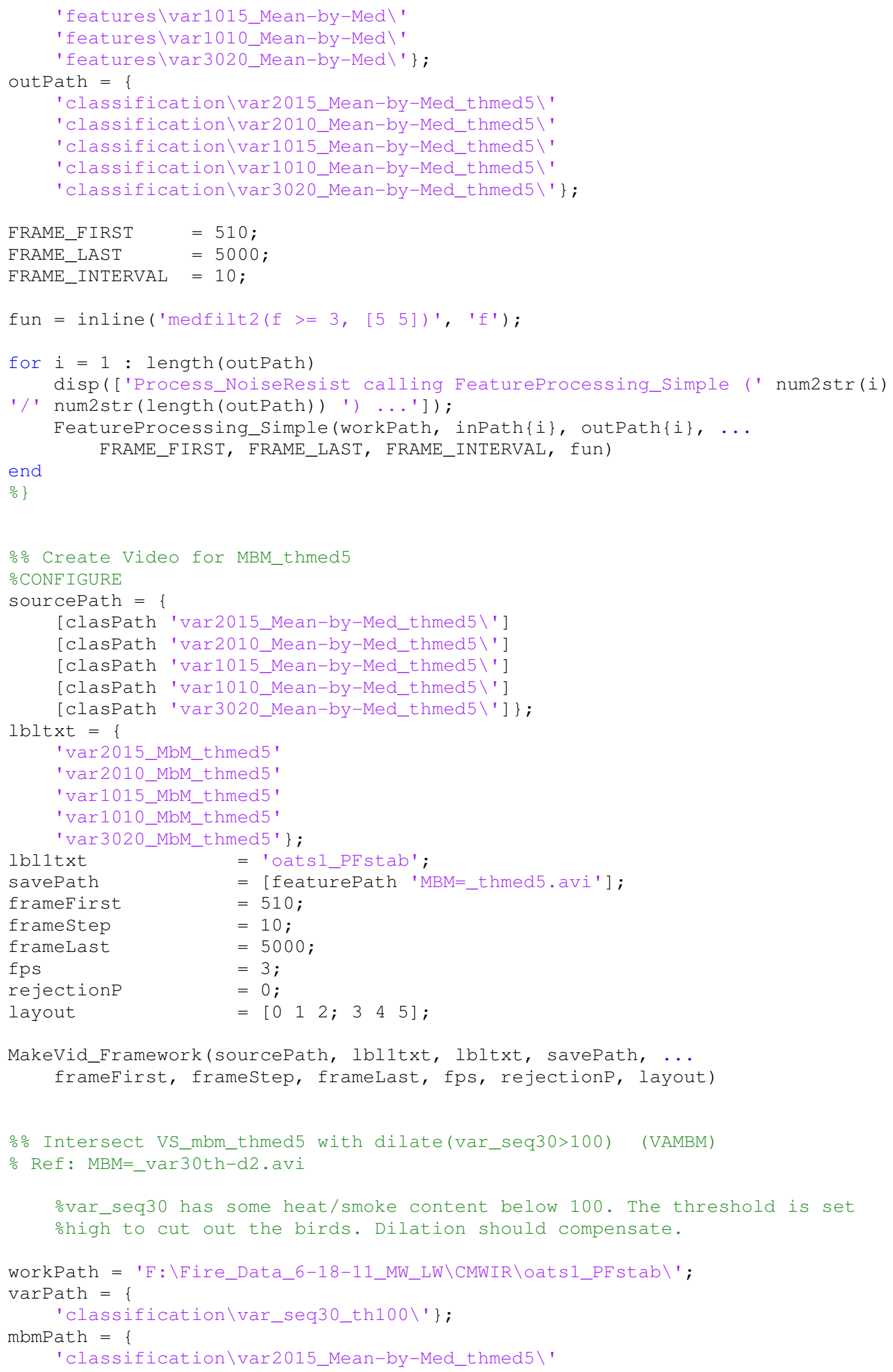




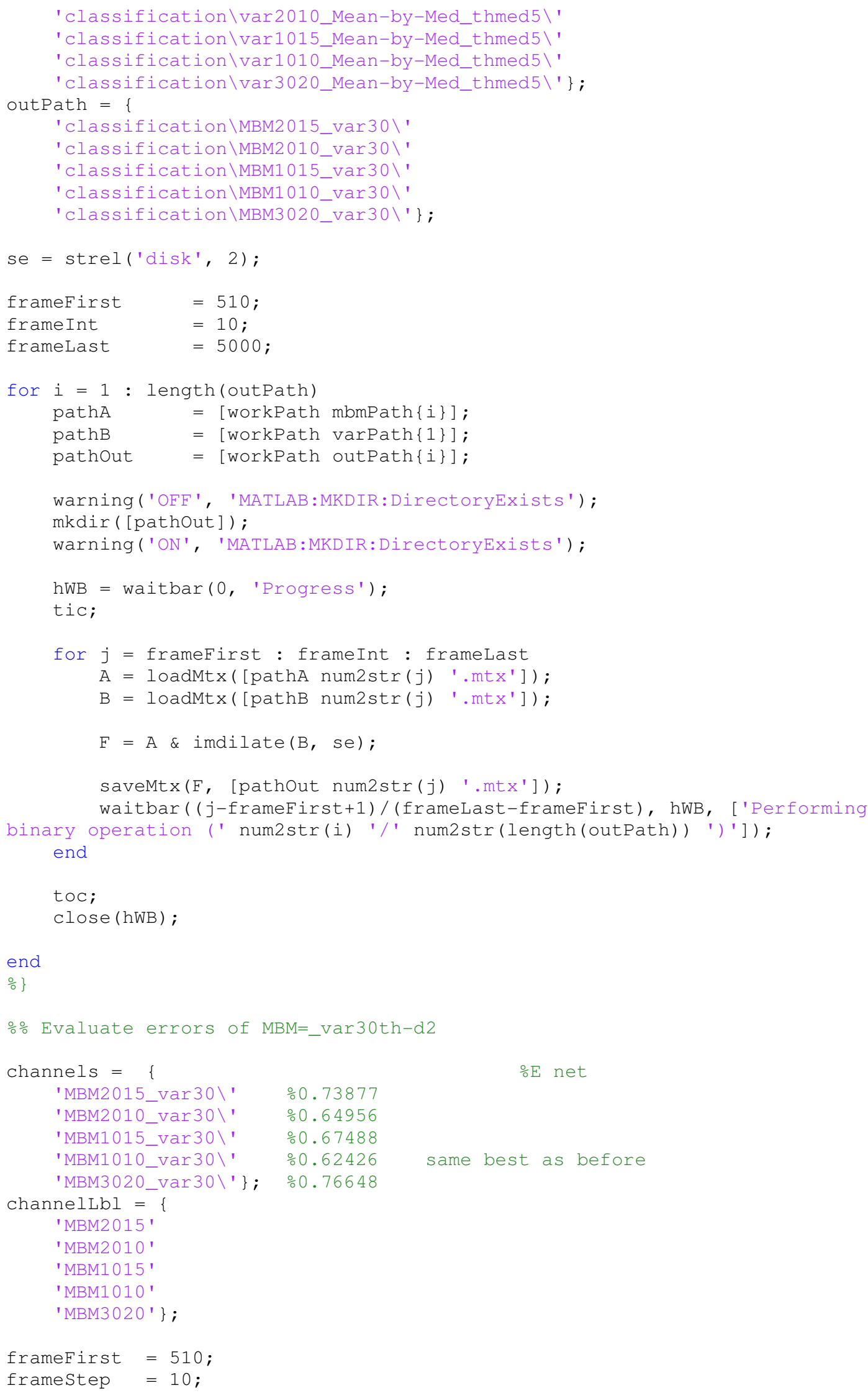




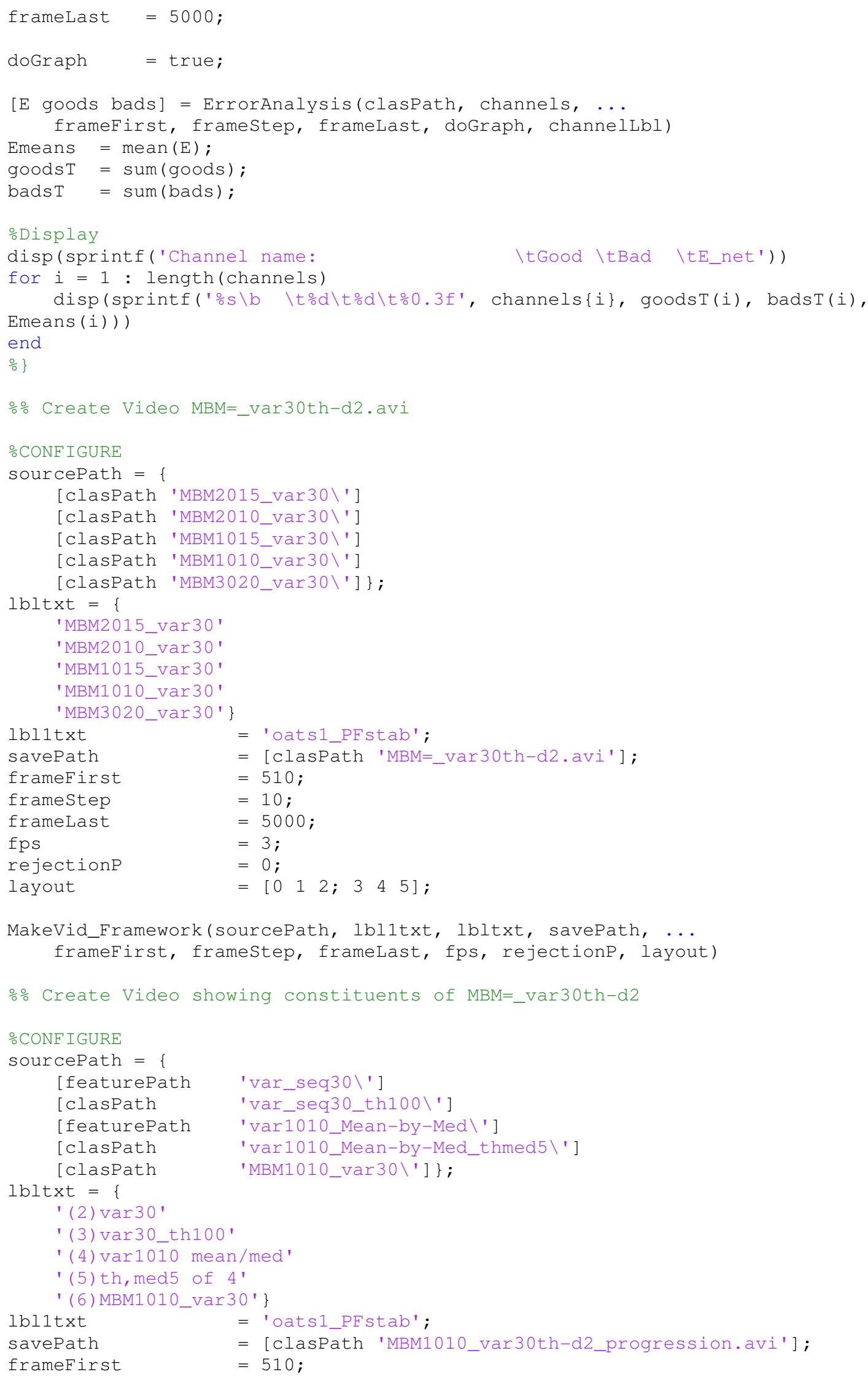




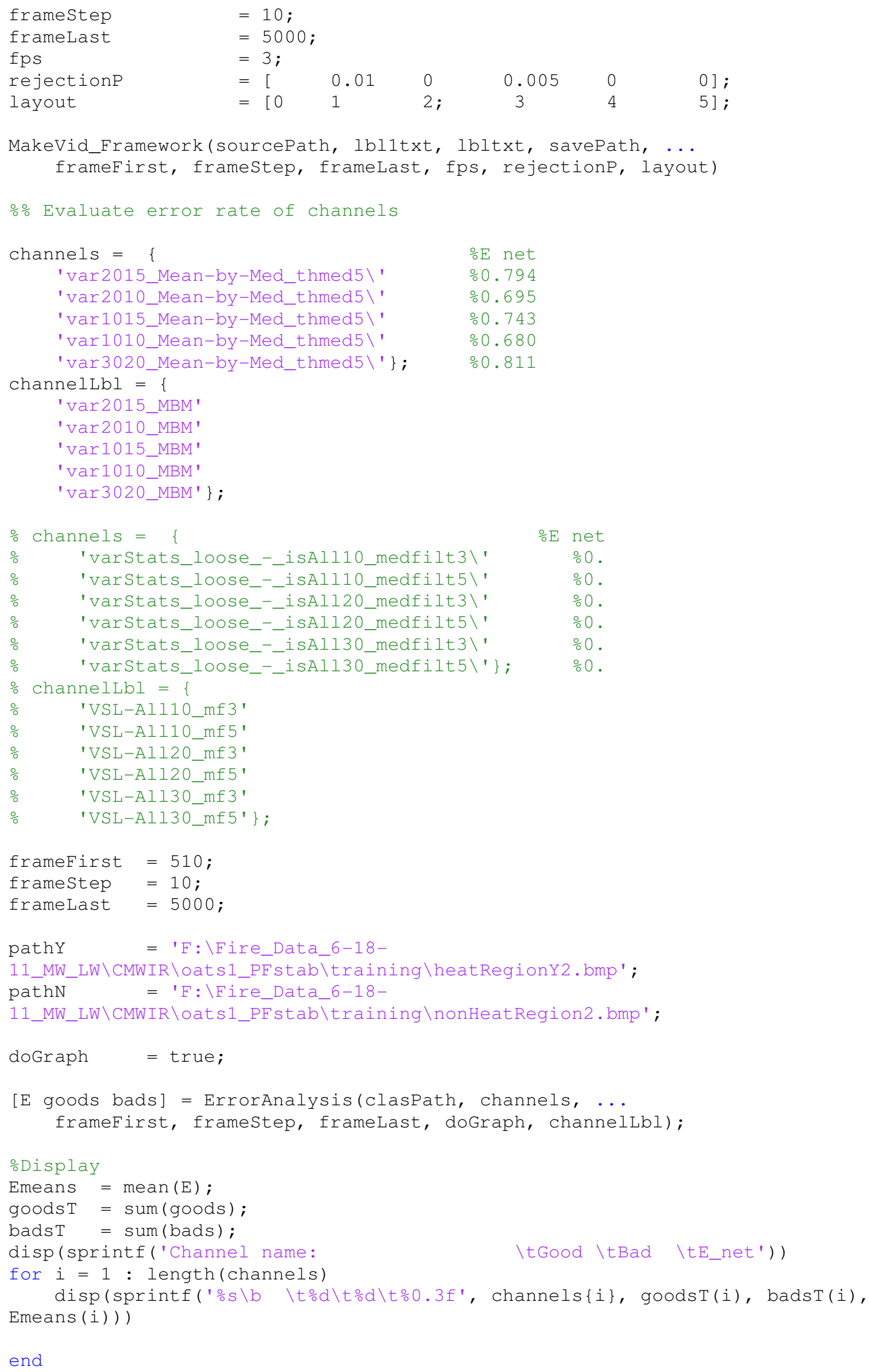




\section{FeatureProcessing_Simple}

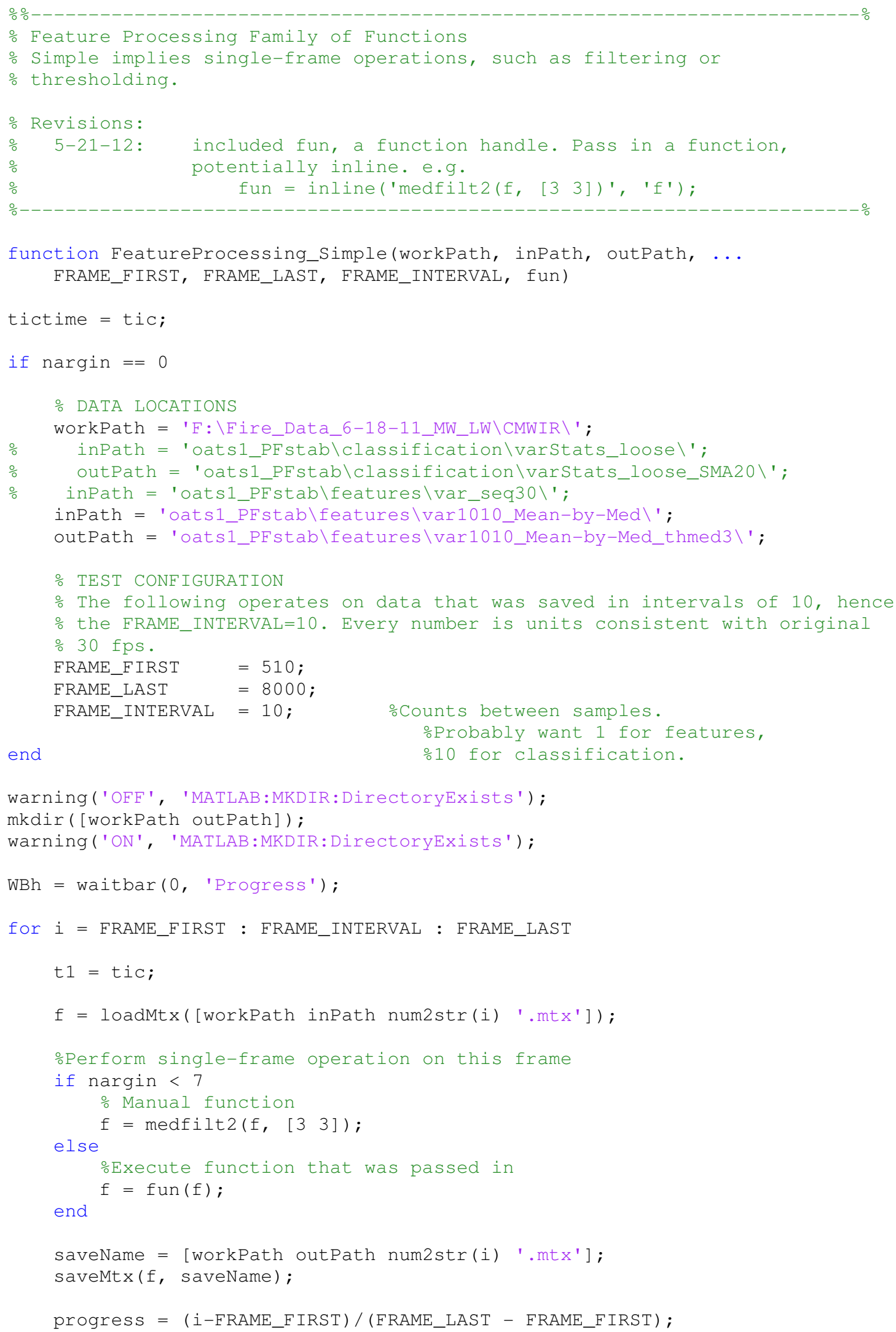


waitbar(progress , WBh, ['Featureprocessing $\backslash$ _Simple: finished i = ' num2str(i) ' in ' num2str(toc(t1)) 's.']);

end $\%$ i (frame groups)

disp(['FeatureProcessing_Simple elapsed time is ' num2str(toc(tictime))]); close (WBh)

halleluja;

end 


\section{Process_DRMAD_PCA.m}

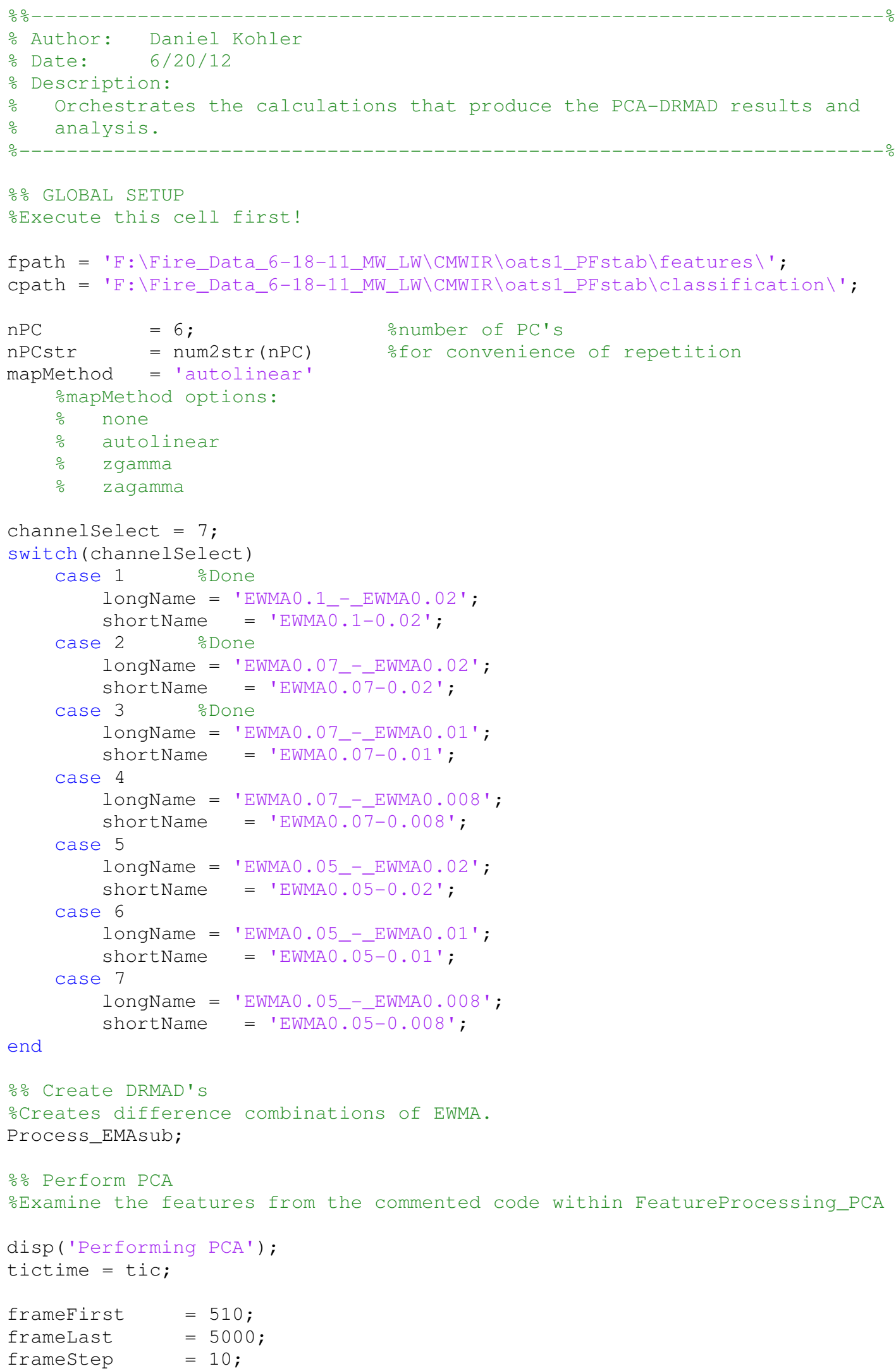




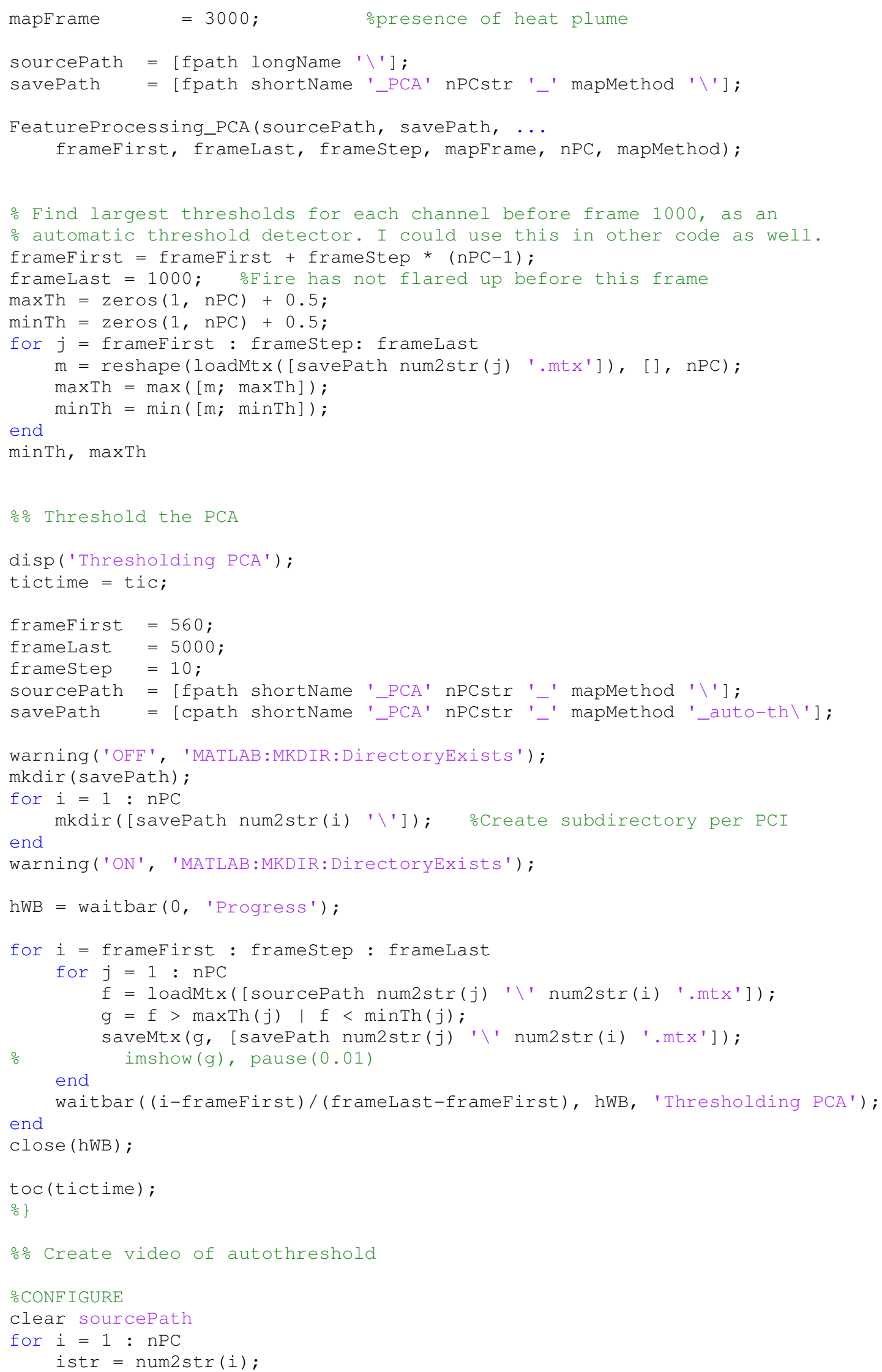




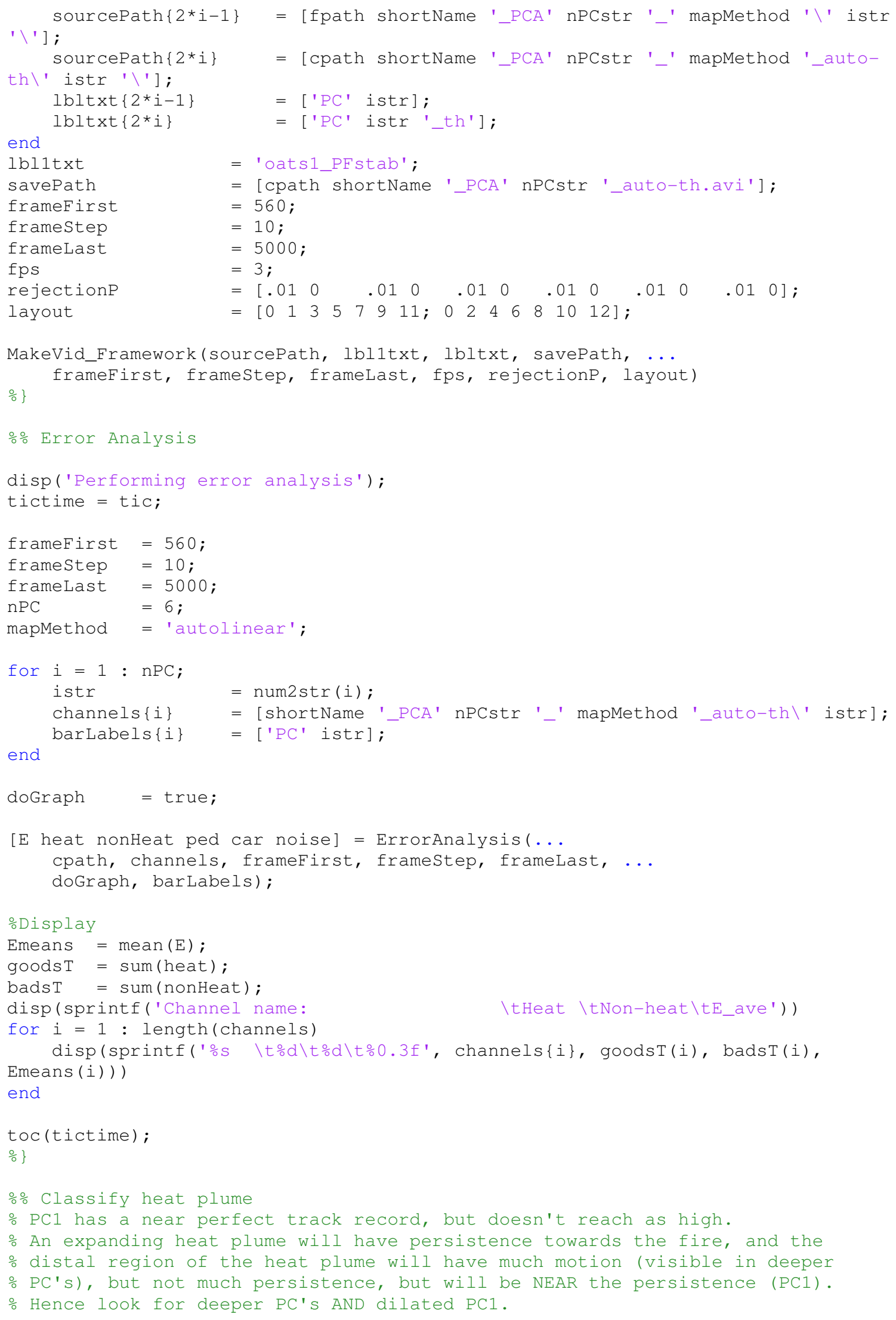




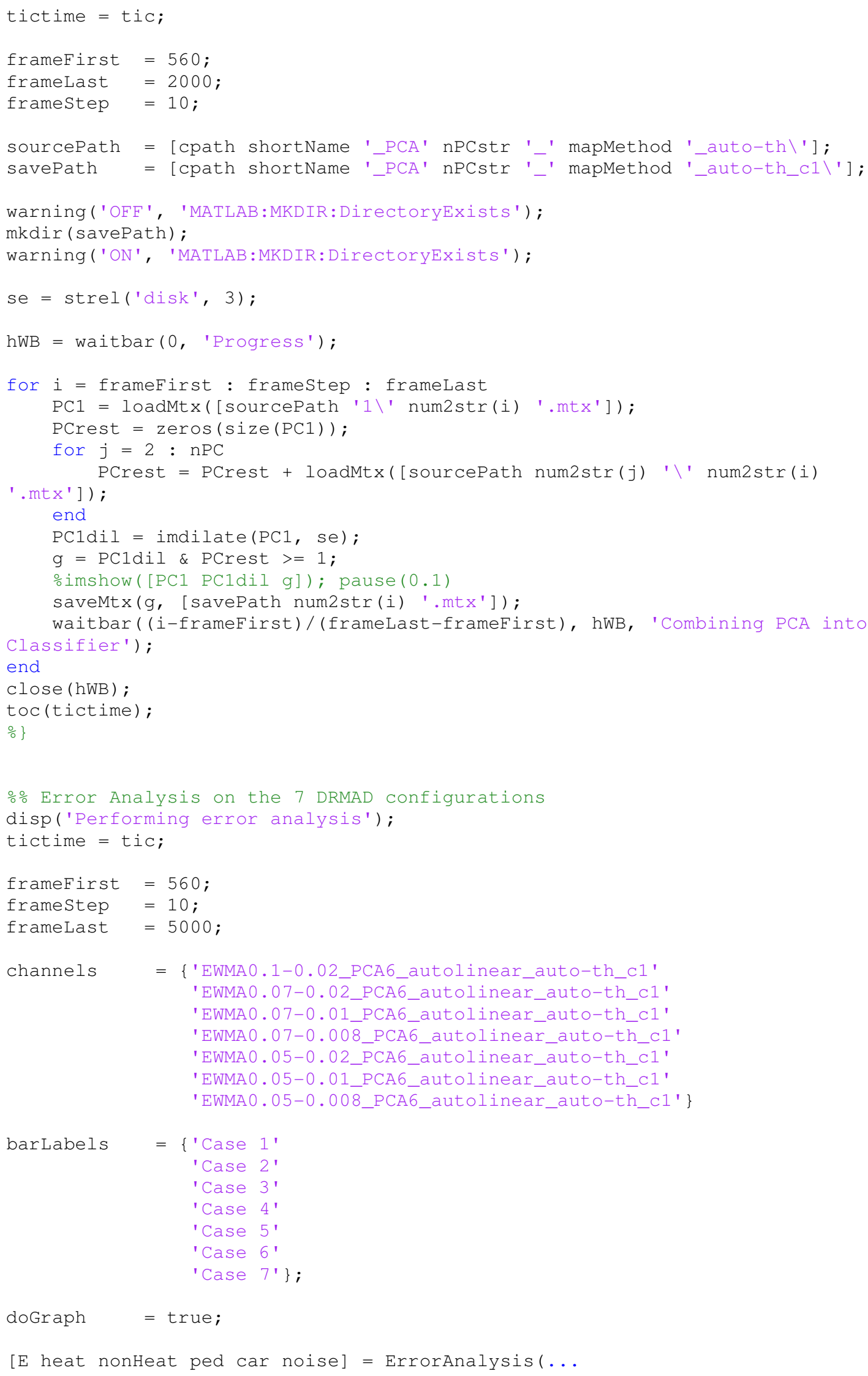


cpath, channels, frameFirst, framestep, framelast, ...

doGraph, barLabels);

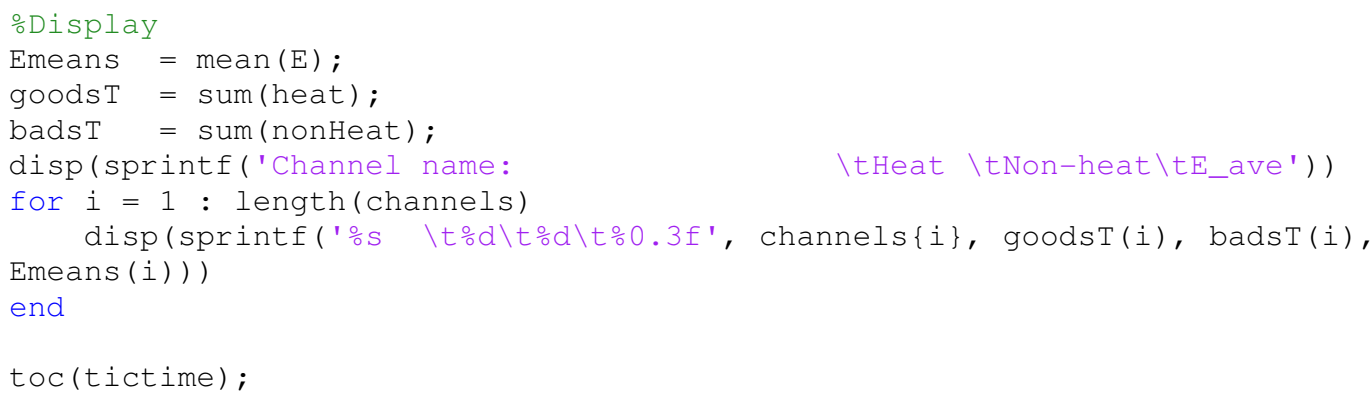




\section{Process_EMAsub.m}

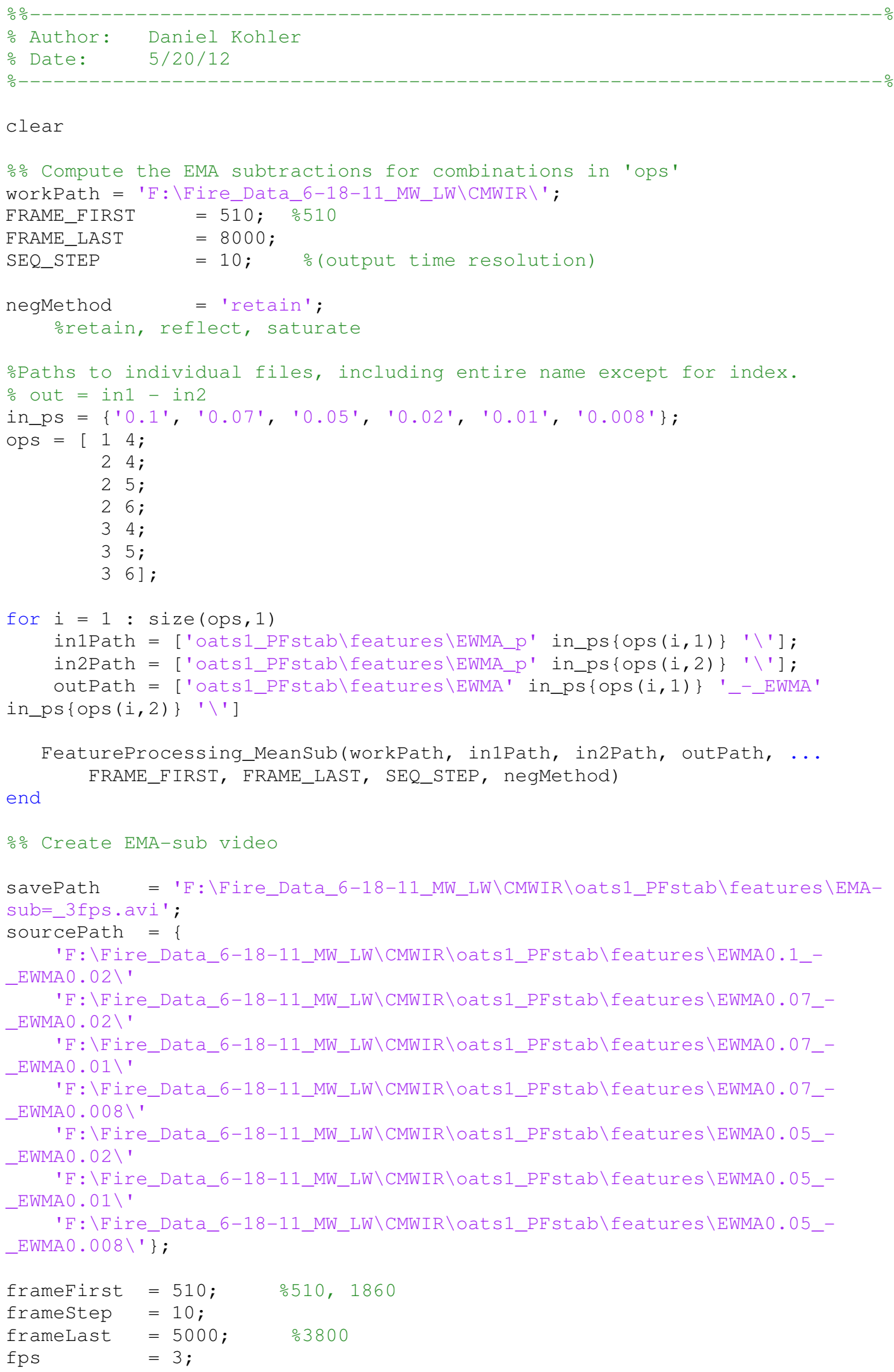




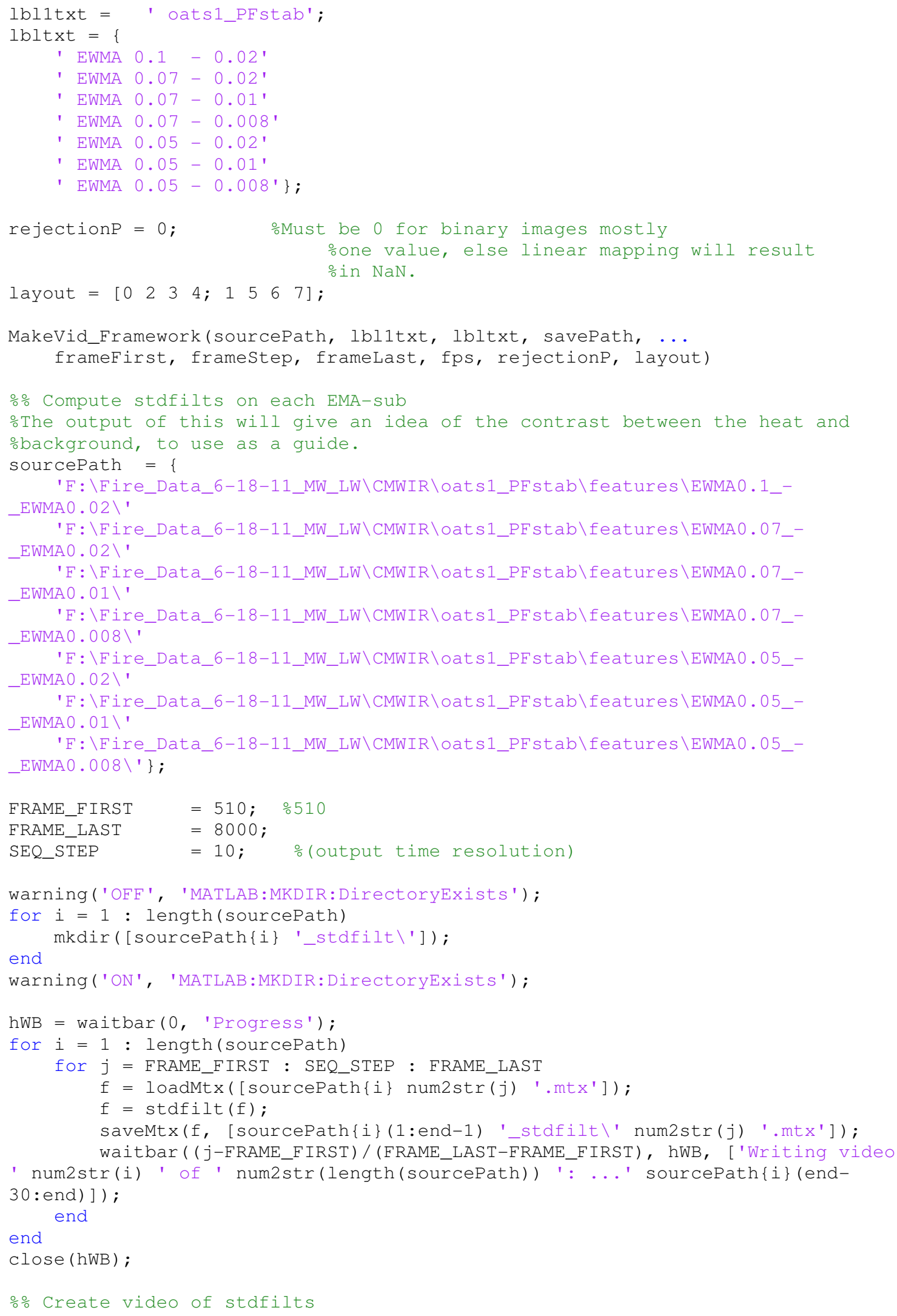




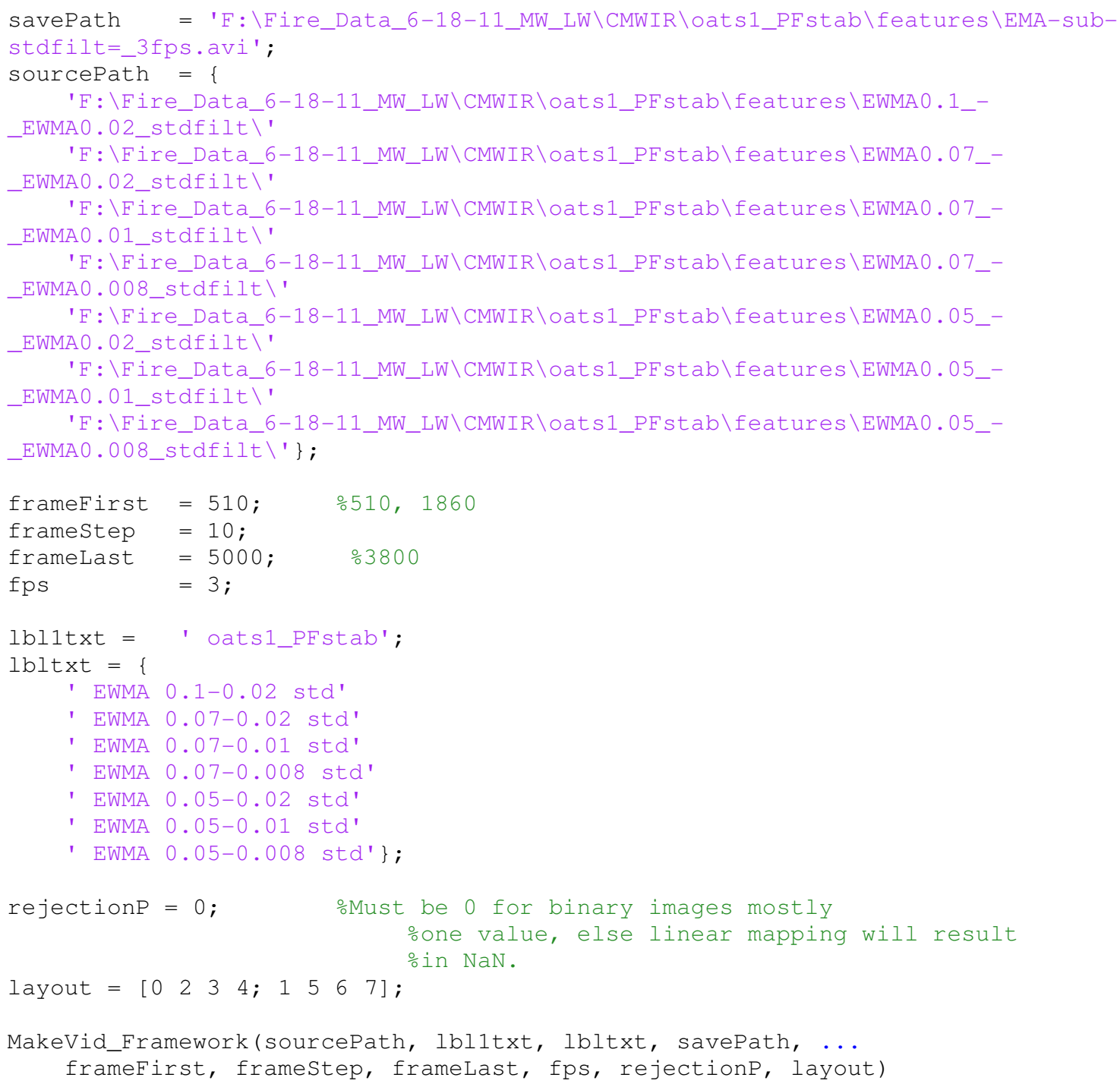




\section{Classification_SVM}

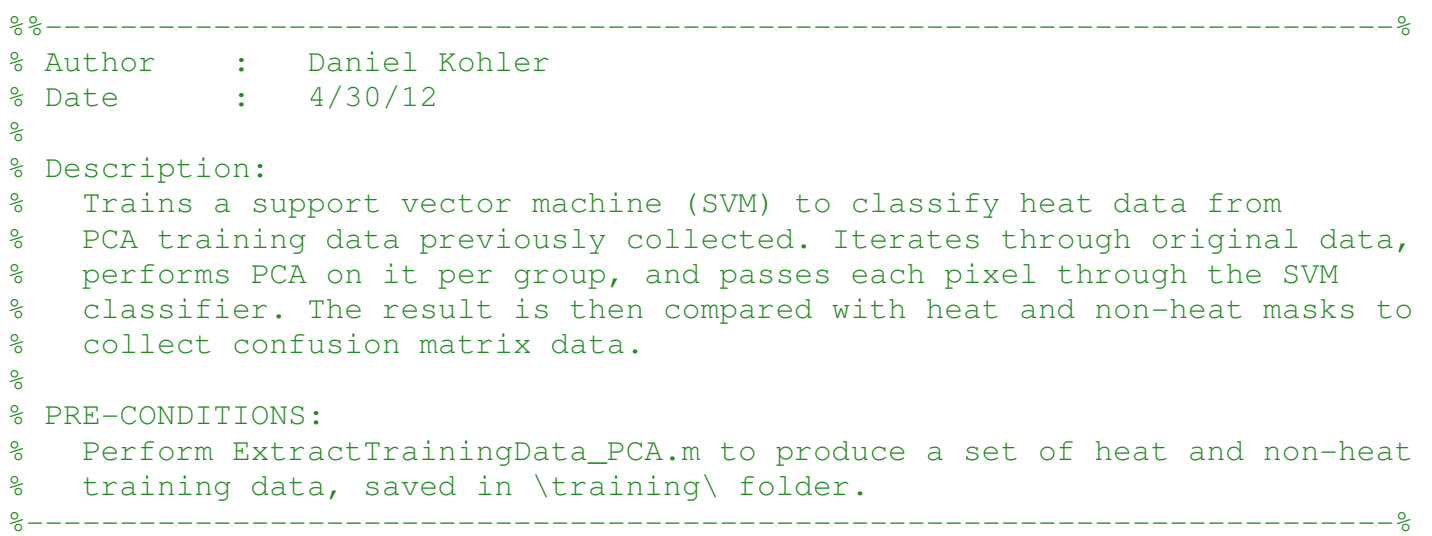


if $(0)$

oTest the SVM with 5-fold stratified cross-validation.

$\mathrm{CV}=$ cvpartition(clas, 'Kfold', 5);

err $=$ zeros (CV.NumTestSets, 1$)$;

for $i=1: C V \cdot$ NumTestsets

SVM = svmtrain(train(CV.training(i), :), clas (CV.training(i)), ...

'kernel_function', 'rbf', ...

'method', 'LS', ...

'autoscale', 'false', ...

'rbf_sigma', 1);

$\mathrm{cl}=\operatorname{svmclassify}(\mathrm{SVM}, \operatorname{train}(\mathrm{CV}$.test $(i),:))$;

end

$\operatorname{err}(i)=\operatorname{sum}(\mathrm{Cl} \sim=\operatorname{clas}(\mathrm{CV} \cdot \operatorname{test}(i)))$;

totalErr $=\operatorname{sum}(\operatorname{err}) / \mathrm{CV} \cdot \mathrm{N}$

oconMat $=$ confusionmat(train (CV.training

end

if $(0)$

oTrain SVM using Least Squares method

o(default SMO only works on 2D input)

$\mathrm{SVM}=$ svmtrain(train, clas, $\ldots$

'kernel_function', 'rbf', ...

'method', 'LS', ...

'autoscale', 'false', ...

'rbf_sigma', 1);

save ([savePath 'trainedSVM.mat'], 'SVM');

else

oOr just load the last trained SVM

SVM = getfield(load([savePath 'trainedSVM.mat']), 'SVM'); end

응 CLASSIFICATION

mapMethod = 'nomap';

frameFirst $\quad=1860$;

framelast $\quad=3800$;

framestep $\quad=10$;

groupstep $\quad=30$;

$\mathrm{nPC} \quad=6 ; \quad$ onumber of $\mathrm{PC}^{\prime} \mathrm{S}$

Region of Interest

rowmin $=175$;

rowmax $=450$;

rowlen $=$ rowmax - rowmin +1 ;

colmin $=250 ;$

colmax $=400 ;$

collen $=$ colmax - colmin +1 ;

frame_group_first $=$ frameFirst $-(\mathrm{nPC}-1)$ * frameStep;

frame_group_last = frameFirst;

oPrepare Principle Component Transform

orig = readframes (workPath, sourceName, ...

frame_group_first, frame_group_last, framestep, ...

NCOLS, NROWS, rowmin, rowlen, colmin, collen);

$\mathrm{T}=$ computePCT (orig);

tic

hWB = waitbar (0, 'Progress');

Prepare confusion matrix

$i=1$; 


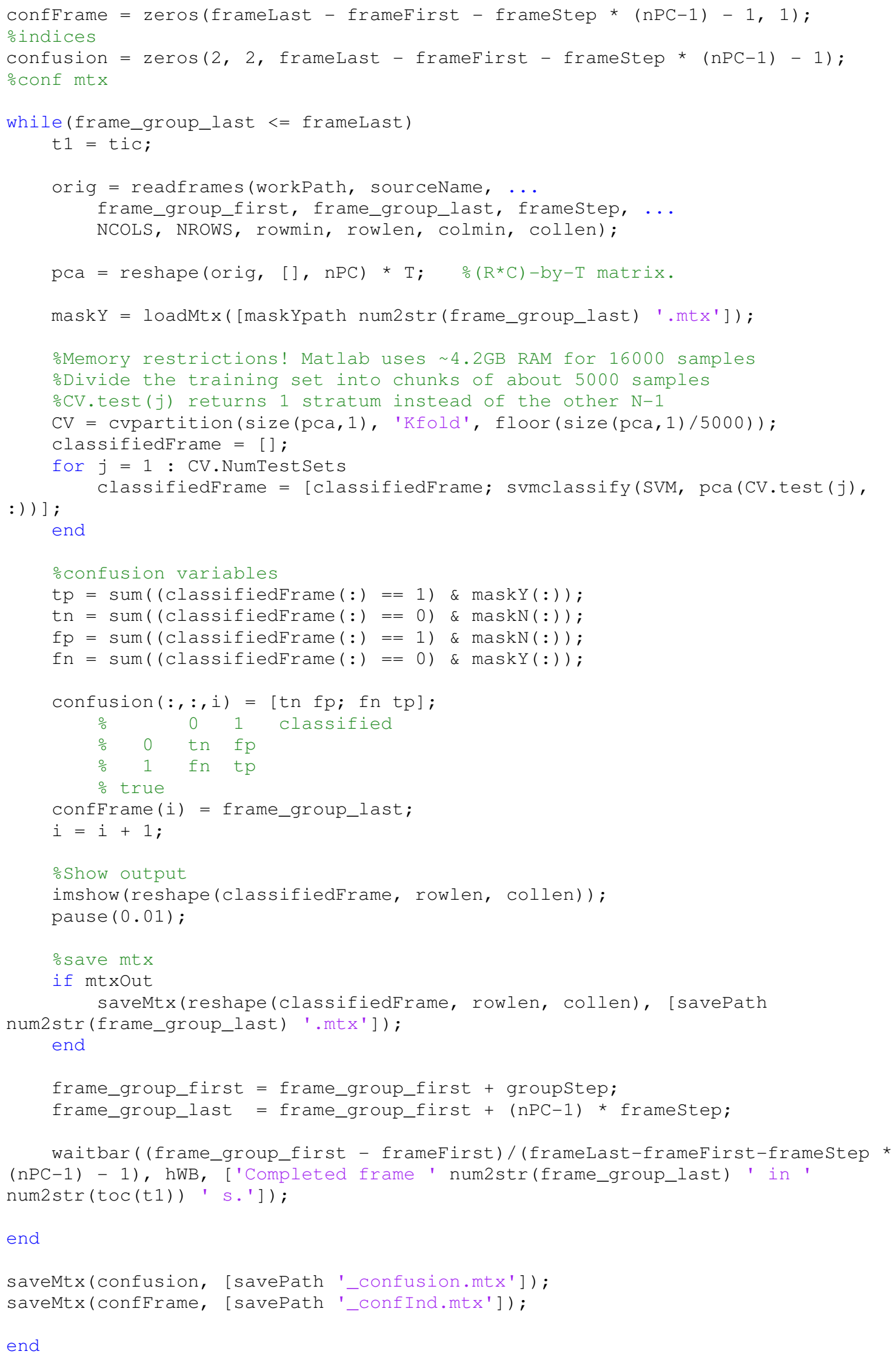




\section{ExtractTrainingData_PCA.m}

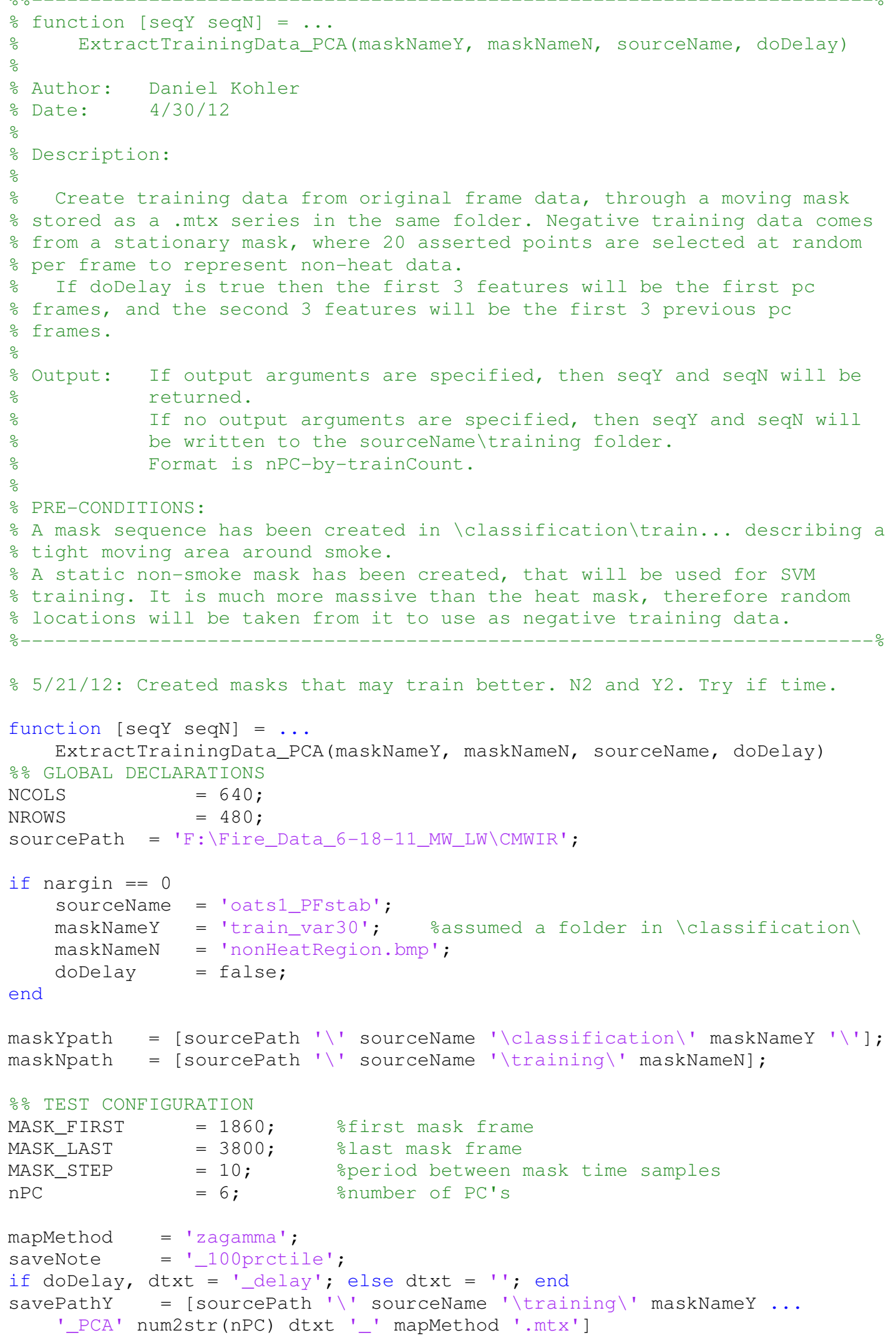




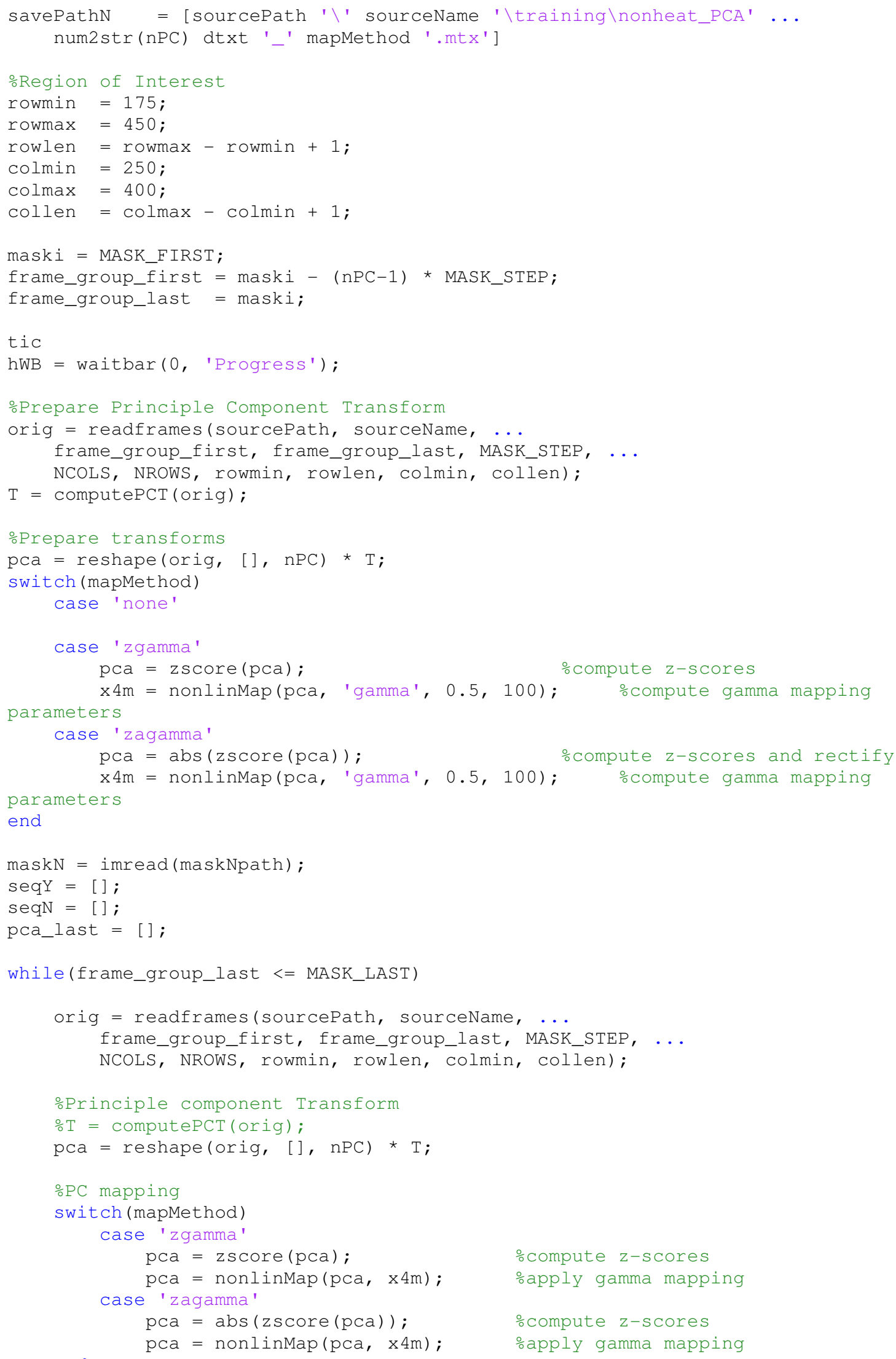




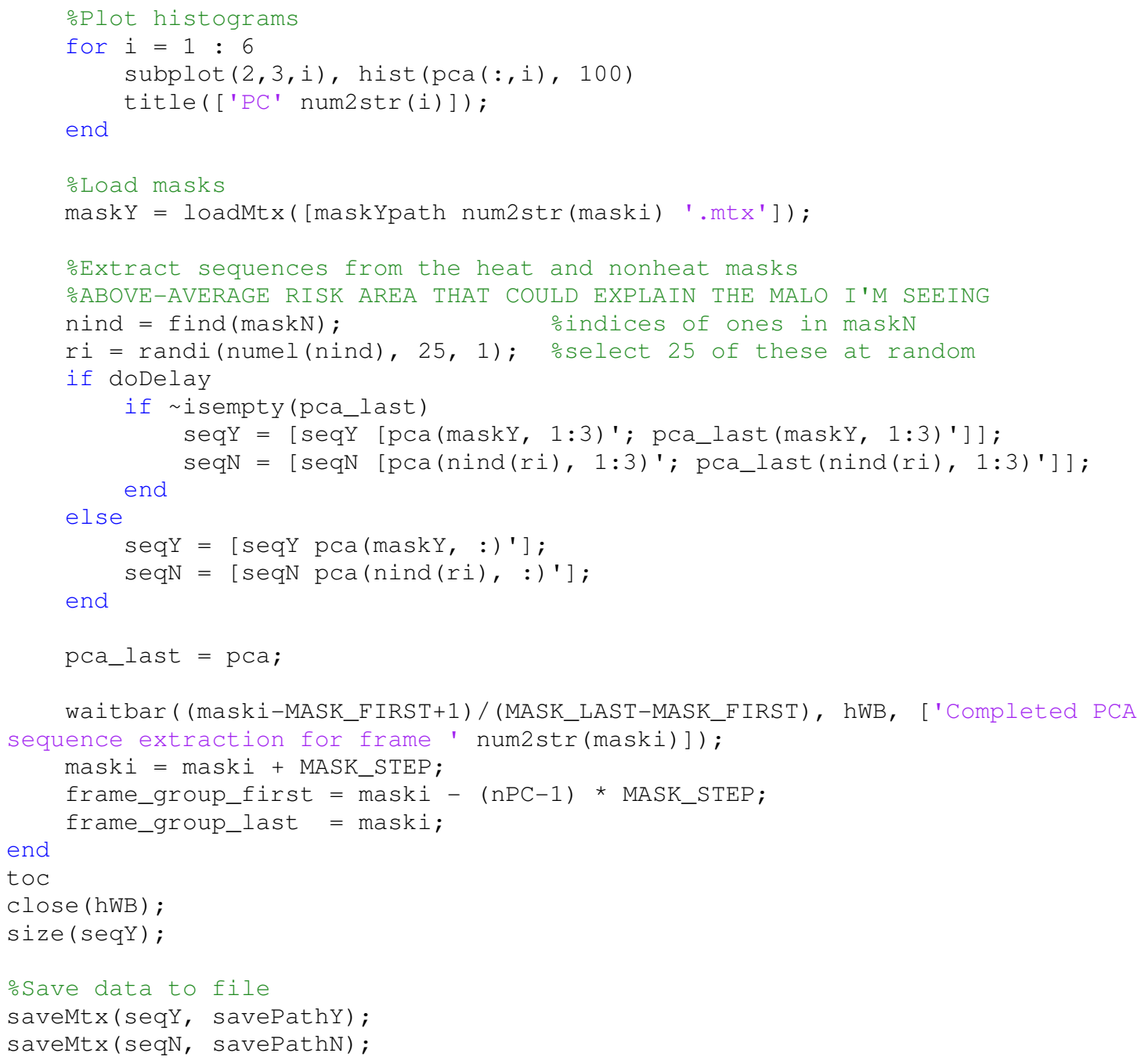




\section{ErrorAnalysis.m}

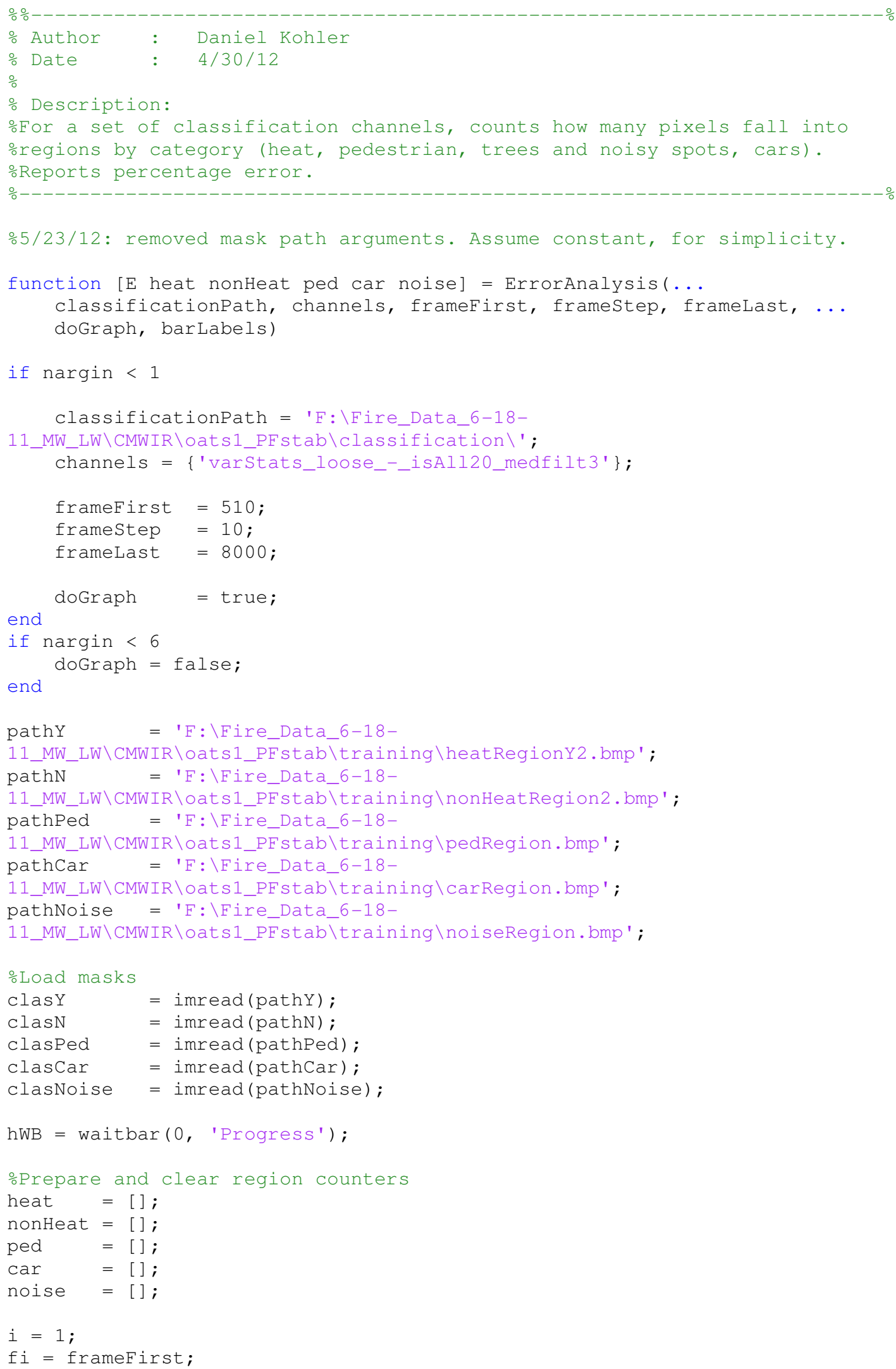




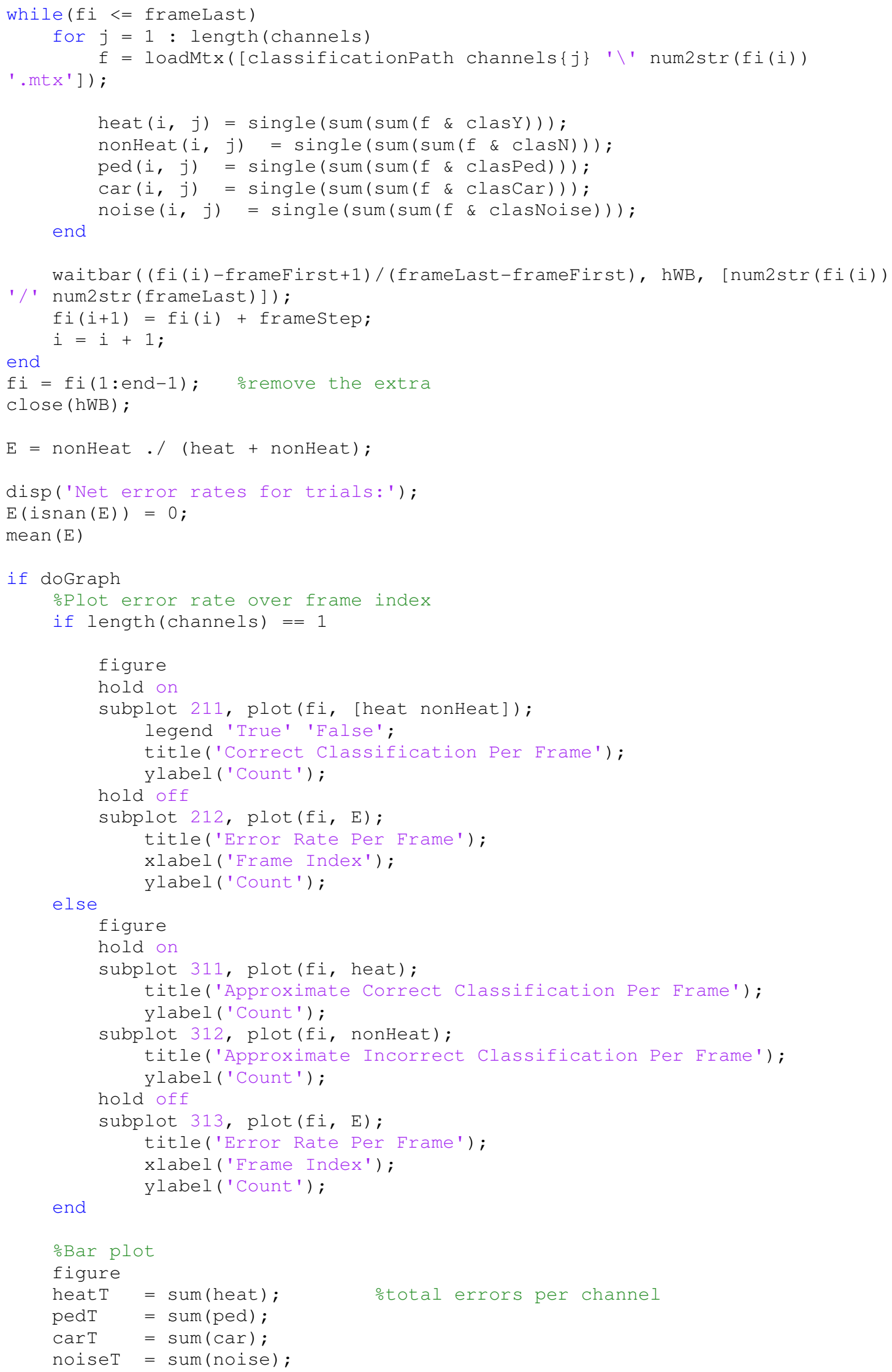




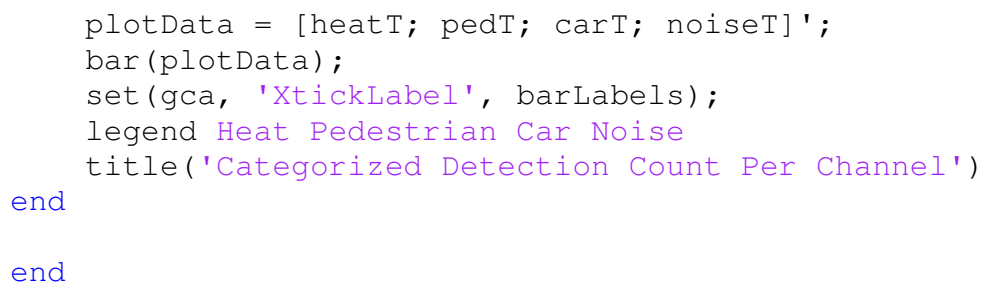

\section{stabilizeVidPFraw.m}

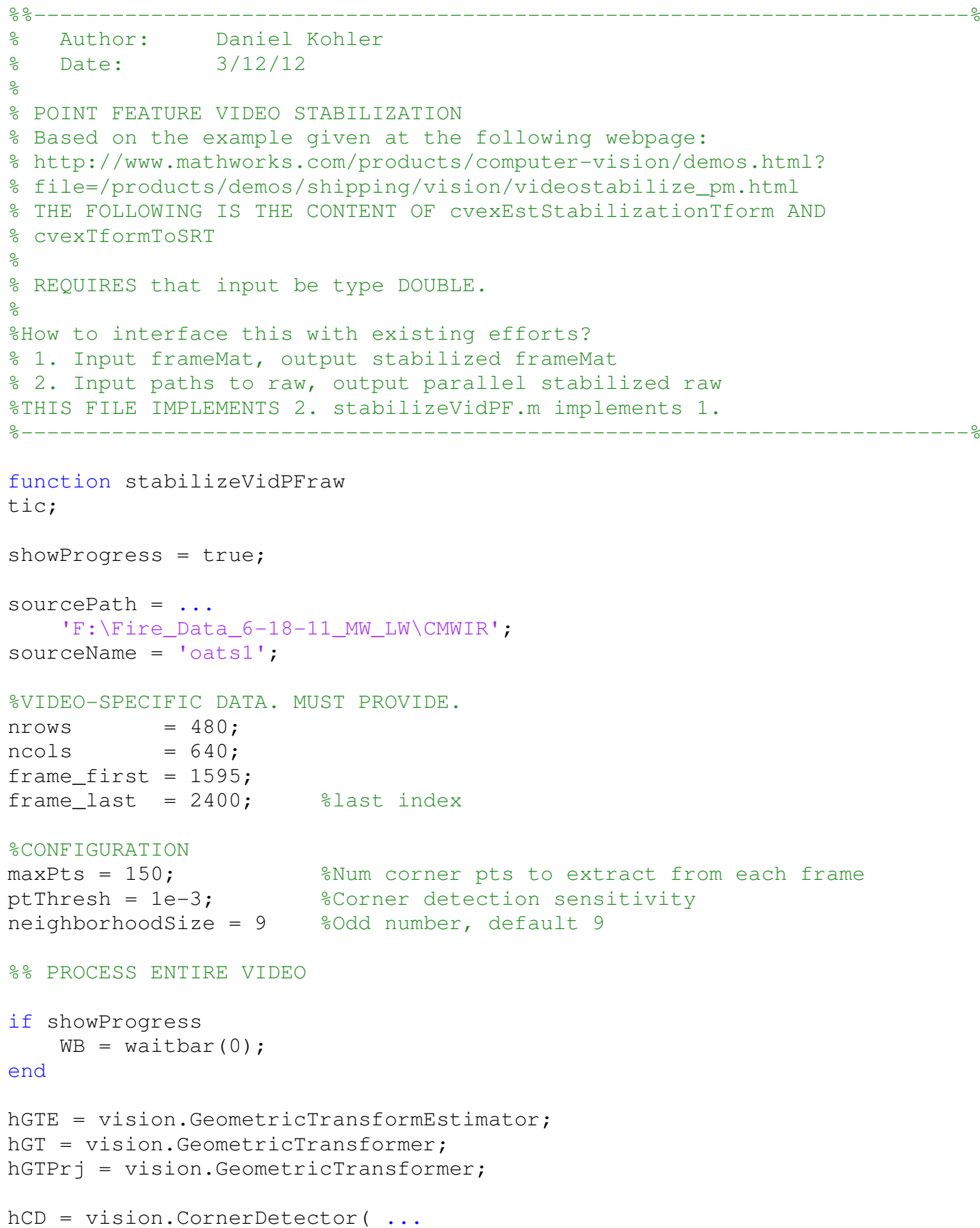




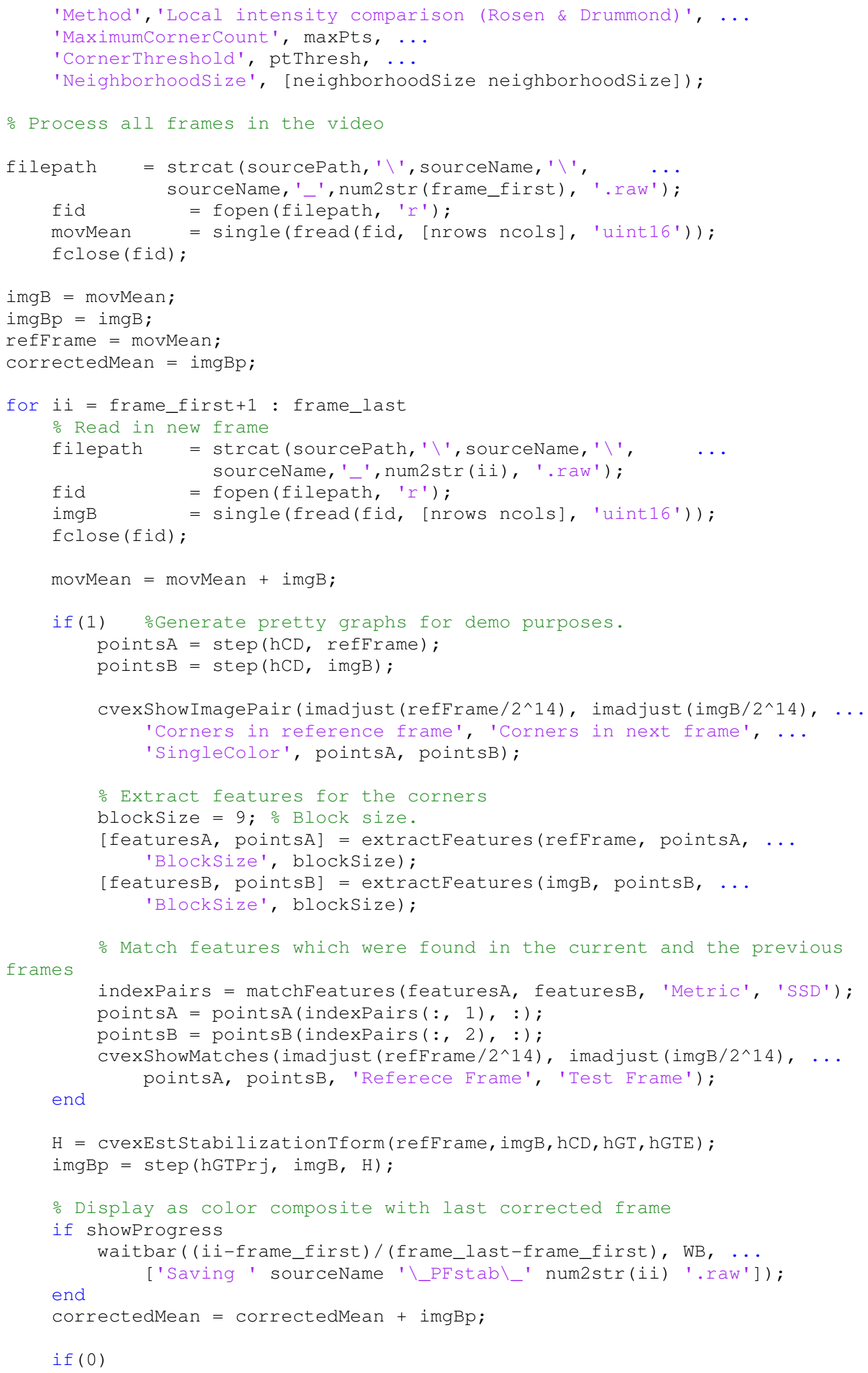




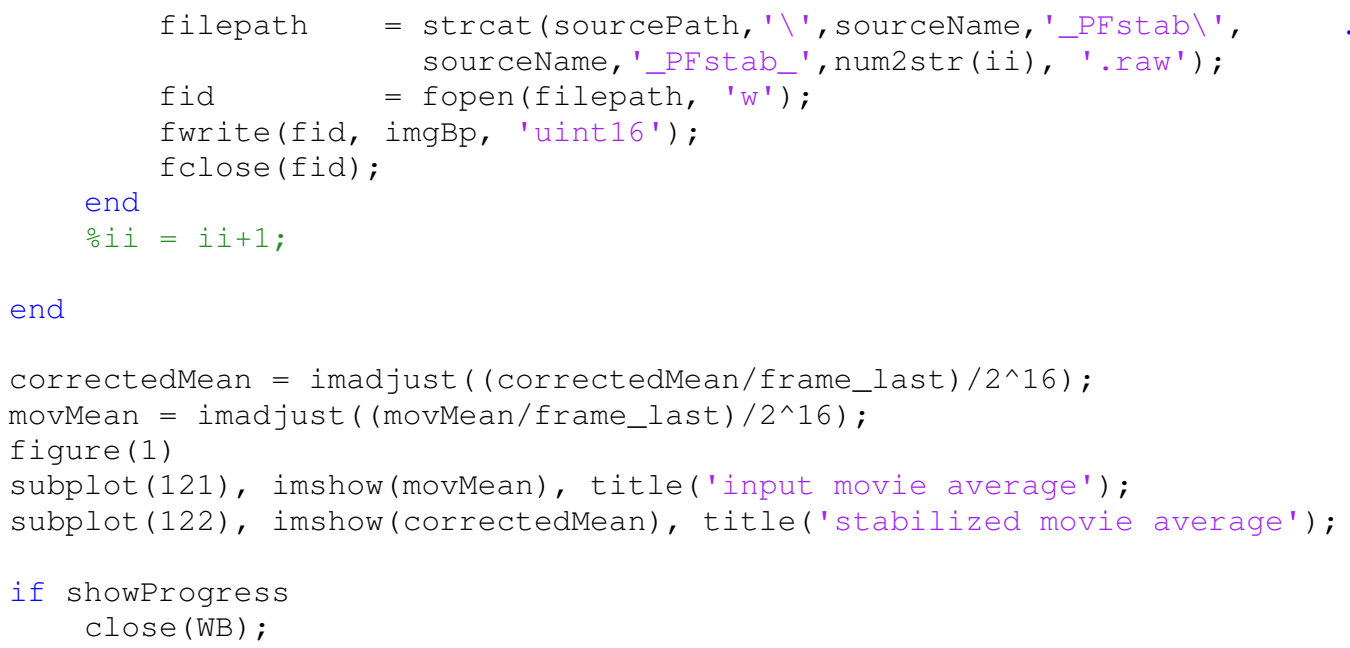

